\title{
Orbiting Clouds of Material at the Keplerian Co-rotation Radius of Rapidly Rotating Low-mass WTTs in Upper Sco
}

\author{
John Stauffer ${ }^{1}$, Andrew Collier Cameron ${ }^{2}$, Moira Jardine ${ }^{2}$, Trevor J. David ${ }^{3}$, Luisa Rebull ${ }^{1,4}$, Ann Marie Cody ${ }^{5}$, \\ Lynne A. Hillenbrand ${ }^{3}$, David Barrado ${ }^{6}$, ${\text { Scott } \text { Wolk }^{7} \text {, James Davenport }}^{8}$, and Marc Pinsonneault ${ }^{9}$ \\ ${ }^{1}$ Spitzer Science Center (SSC), California Institute of Technology, Pasadena, CA 91125, USA; stauffer@ipac.caltech.edu \\ ${ }^{2}$ School of Physics and Astronomy, University of St. Andrews, North Haugh, St Andrews KY16922, UK \\ ${ }_{3}^{3}$ Astronomy Department, California Institute of Technology, Pasadena, CA 91125, USA \\ ${ }^{4}$ Infrared Science Archive (IRSA), 1200 E. California Blvd, MS 314-6, California Institute of Technology, Pasadena, CA 91125, USA \\ ${ }^{5}$ NASA Ames Research Center, Space Sciences and Astrobiology Division, MS245-3, Moffett Field, CA 94035 USA \\ ${ }^{6}$ Centro de Astrobiología, Dpto. de Astrofísica, INTA-CSIC, E-28692, ESAC Campus, Villanueva de la Cañada, Madrid, Spain \\ ${ }^{7}$ Harvard-Smithsonian Center for Astrophysics, 60 Garden Street, Cambridge, MA 02138, USA \\ ${ }^{8}$ Department of Physics \& Astronomy, Western Washington University, 516 High Street, Bellingham, WA 98225, USA \\ ${ }^{9}$ Department of Astronomy, The Ohio State University, Columbus, OH 43210, USA \\ Received 2016 November 3; revised 2017 January 30; accepted 2017 February 3; published 2017 March 10
}

\begin{abstract}
Using $K 2$ data, we identified 23 very-low-mass members of the $\rho$ Oph and Upper Scorpius star-forming region as having periodic photometric variability not easily explained by well-established physical mechanisms such as star spots, eclipsing binaries, or pulsation. All of these unusual stars are mid-to-late $\mathrm{M}$ dwarfs without evidence of active accretion, and with photometric periods generally $<1$ day. Often the unusual light-curve signature takes the form of narrow flux dips; when we also have rotation periods from star spots, the two periods agree, suggesting that the flux dips are due to material orbiting the star at the Keplerian co-rotation radius. We sometimes see "statechanges" in the phased light-curve morphologies where $\sim 25 \%$ of the waveform changes shape on timescales less than a day; often, the "state-change" takes place immediately after a strong flare. For the group of stars with these sudden light-curve morphology shifts, we attribute their flux dips as most probably arising from eclipses of warm coronal gas clouds, analagous to the slingshot prominences postulated to explain transient $\mathrm{H} \alpha$ absorption features in AB Doradus and other rapidly rotating late-type stars. For another group of stars with somewhat longer periods, we find the short-duration flux dips to be highly variable on both short and long timescales, with generally asymmetric flux-dip profiles. We believe that these flux dips are due to particulate clouds possibly associated with a close-in planet or resulting from a recent collisional event.
\end{abstract}

Key words: stars: low-mass - stars: pre-main sequence

\section{Introduction}

We have entered into a new era, where synoptic photometry for millions of stars is currently being collected and analyzed (e.g., OGLE, Gaia, WASP). In the relatively near future, LSST will obtain synoptic photometry for billions of stars. In order to make the most of those data, we need to develop automated routines to sift through enormous data sets in order to identify light curves of interest. A precursor to that activity is to develop a better understanding of all the types of photometric variability present in real stars and, where possible, to assign physical mechanisms to those variability classes.

The Kepler mission provided one very well characterized training set of light curves for $>100,000$ stars. However, because it was tied to a single position in the sky and the target stars were primarily chosen for one science goal, the Kepler data only provide templates for a limited range of stellar variability types. The $K 2$ mission (Howell et al. 2014) is providing a greatly augmented set of data by observing a new group of $\sim 20,000$ stars every three months, with those stars having a much wider range of characteristics. One specific variability type for which $K 2$ is providing a wealth of new data is young stellar objects (YSOs).

During Campaign 2 of the $K 2$ mission, nearly 1500 candidate members of the star-forming regions comprised of the $\rho$ Oph dark-cloud complex and the Upper Scorpius association were targeted. The data from that 78 -day campaign form the largest set of precision YSO optical light curves that have ever been obtained. Those light curves provide a hunting ground for the search for newly formed planets via their transits (David et al. 2016b; Mann et al. 2016), for the identification of YSO eclipsing binaries (Alonso et al. 2015; Kraus et al. 2015; Lodieu et al. 2015; David et al. 2016a), and for the determination of the rotational periods for a large sample of low-mass stars of 5-10 Myr age (L. Rebull et al. 2017, in preparation). A small fraction of the Upper Sco YSOs have IR excesses and are likely classical T Tauri stars (CTTs); a subgroup of "burster" light curves associated with some of these accreting systems is discussed in Cody et al. (2017).

The discussion of our process for identifying a best set of probable Upper Sco members, and the categorization of lightcurve morphologies for the disked stars is provided in A. M. Cody et al. (2017, in preparation). Our sorting of those members into stars with primordial disks and those without disks (weak-lined T Tauri stars, WTTs) is described briefly below, and more fully in A. M. Cody et al. (2017, in preparation).

About 1100 of the Upper Sco and $\rho$ Oph stars appear not to have infrared (IR) excesses and presumably have shed their primordial circumstellar disks. In the course of our processing and analysis of those light curves, the vast majority were identified as showing periodic variability characteristic of stars with dark, non-axisymmetrically distributed spots. A handful were found to be eclipsing binaries, and another handful (all 
relatively high-mass stars) are pulsators. However, 23 of the stars showed periodic photometric variability which did not fit into any of those categories. It is those stars that are the subject of this paper. All of these stars are M dwarfs, with most having spectral types between M4 and M6. All have short periodsmore than half less than one day, and all but one less than two days.

In Section 2, we describe the sources of data for this paper. Our process for identifying unusual light-curve classes and the working definitions we have developed for our three classes is provided in Section 3. The empirical characteristics of the variability for each of the three classes is laid out in Sections 4-6.

\section{Observational Data}

\subsection{K2 Light Curves}

For the first three $K 2$ campaigns, the $K 2$ Science Center did not produce detrended light curves. Instead, what was provided was the raw CCD pixel data for each star at each epoch. We (Cody et al. 2017) therefore created our own detrended light curves. We produced two primary versions-one where the aperture centers were held fixed over the course of the $K 2$ campaign and another where a new center was derived at each epoch. For each flavor of reduction, photometry was produced with 2- and 3-pixel radius apertures. For each of these four light-curve versions, we then applied a version of the detrending routine described in Aigrain et al. (2016) to remove the short-term, non-astrophysical trends in the data.

After we had begun writing this paper, two other light-curve reductions became publicly available for Campaign 2, the Vanderburg reduction (Vanderburg \& Johnson 2014) and the EVEREST reduction (Luger et al. 2016). We downloaded light curves for each of the stars that we considered as possibly relevant to this paper.

For each star addressed in this paper, we examined all of the light-curve variants that we had available and chose the one that appeared to have the least non-astrophysical contribution (when several variants were about the same, we simply adopted our own detrended light curve). In all cases, the unusual features that caused us to include the star in our paper were present in multiple light-curve variants. In a few cases, one of the light-curve variants did not have the same signature. We believe this indicates that the detrending algorithm employed in that version removed some real astrophysical signature, or that the aperture size used in that version or the centering algorithm in that version resulted in the light curve being dominated by light from a different star. We made special effort in these cases to closely examine all the available data (finding charts, literature, all light-curve variants, and different aperture sizes) in order to make sure that the light curve we present here is the best available for that star.

\subsection{Ancillary Data}

The Ecliptic Plane Input Catalog (EPIC; Huber et al. 2016), available at MAST, provides accurate coordinates for all of the stars in our sample. Using those coordinates, we downloaded all available near and mid-IR photometry for our stars from the 2MASS (Skrutskie et al. 2006), WISE (Wright et al. 2010),
Spitzer (Werner et al. 2004) SEIP $^{10}$ and FEPS (Meyer et al. 2006), AKARI (Murakami et al. 2007), and SDSS (e.g., Ahn et al. 2014) archives as well as $B$ - and $V$-band photometry from APASS (Henden \& Munari 2014). We also downloaded grizy photometry for most of our stars from the PAN-STARRS database (Flewelling et al. 2016). Spectral types were available in the literature (Preibisch et al. 2002; Erickson et al. 2011; Lodieu et al. 2011; Luhman \& Mamajek 2012; Rizzuto et al. 2015 ) for $80 \%$ of our stars; for most of the stars, those same references also provided $\mathrm{H} \alpha$ and lithium $(6708 \AA)$ equivalent widths. We have used our own Keck HIRES spectra to provide a small amount of new spectral information, as described in the Appendix.

One goal for the ancillary data was to provide a colormagnitude diagram that would include all of the stars in our sample, and for that we decided to attempt to determine estimated $V-K_{s}$ colors for every star. Because only a small fraction of the Upper Sco members have published $V$-band photometry, this required transforming photometry or colors obtained at other optical bands. As the first step in that process, we downloaded $G$-band photometry for all of our stars from the newly released Gaia DR1 archive (Gaia Collaboration et al. 2016). We then derived a formula to convert $G-K_{s}$ colors to $V-K_{s}$ colors based on a large sample of low-mass stars with published $V-K_{s}$ colors in Praesepe (L. Rebull et al. 2017, in preparation). For the stars lacking Gaia photometry, we obtained I magnitudes from the Deep Near-Infrared Survey of the Southern Sky (DENIS; Epchtein et al. 1997) and derived a conversion formula in a similar way between $I_{\text {(DENIS) }}-K_{s}$ and $V-K_{s}$.

We used all of the available photometry and spectral types to produce spectral energy distributions (SEDs) for each star (the individual SEDs are shown in the Appendix). From those SEDs, we identified stars with possible IR excesses. For each of the sources thought to have an IR excess, we looked at the images, where available, to assess the quality of the photometry. The longest wavelength data we have is either WISE $22 \mu \mathrm{m}$ or, for just four stars Spitzer $24 \mu \mathrm{m}$. In several cases, the $22 \mu \mathrm{m}$ point from WISE, in particular, was not a secure detection, and the apparent IR excess therefore vanished. Only one star in our final sample has a relatively secure, though small IR excess (only at $24 \mu \mathrm{m}$ ), and that is EPIC 205024957. Carpenter et al. (2009) also determined that EPIC 205024957 has a $24 \mu \mathrm{m}$ excess and identified it as a debris disk. For the stars where our longest wavelength detection is at $12 \mu \mathrm{m}$, the lack of an excess at that wavelength precludes them from having primordial disks. Because of the paucity of good data longward of $12 \mu \mathrm{m}$ for most of our stars, a larger fraction could harbor debris disks.

\section{Sample Selection and Light-curve Taxonomy}

\subsection{Sample Selection}

The first step in our sample selection was to eliminate stars with active accretion (CTTs) from the Upper Sco and $\rho$ Oph $K 2$ sample, since the accretion process in CTTs and interactions with the primordial circumstellar disk can produce unusual light curves of many types, and addressing those types of variabilities was beyond the scope we wanted here (but see Cody et al. 2017). None of the stars we retained

\footnotetext{
${ }^{10}$ http://irsa.ipac.caltech.edu/data/SPITZER/Enhanced/SEIP/ overview.html
} 
for this paper have SEDs consistent with Class I or II primordial disks (see the SED plots in the Appendix, and compare, for example, to the SEDs of Class I and II sources in Upper Sco shown in Cody et al. 2017 or similar sources in Taurus, as shown in Rebull et al. 2010). Two of the stars are considered as $\rho$ Oph members in the literature (EPIC 203962559 and EPIC 203927435); all of the others are considered as members of Upper Sco.

Once we identified the full set of Upper Sco weak-lined T Tauri (WTTS) members with $K 2$ data, three of the authors (T.J.D., L.M.R., A.M.C.) independently examined the data for each star, including each of the light-curve variants described above. They also independently searched for periodicities in those light curves and examined the phased light curves. Those efforts were aimed primarily at identifying possible planet transits, eclipsing binary candidates, or variability related to accretion or circumstellar disk structure or in compiling stellar rotation periods. During the course of those efforts, a few dozen stars were identified as having light curves that were not easily explained by any of those mechanisms. In those cases, further analysis was done and other members of the team were brought in to perform more manual detrending of the light curves. This more detailed analysis resulted in some of these light curves being ascribed to artifacts in the data or to allowing them to fit into one of the established light-curve classes. However, 23 of these stars survived. These stars are listed in Table 1.

\subsection{Definition of Light-curve Classes and Their Morphologies}

We have sorted our unusual light curves into three classes in order to facilitate our discussion of their properties. We acknowledge that this sorting is probably imperfect, and that the boundaries between classes are indistinct. An alternative view is that all of our stars belong to a single group and their differing light-curve morphologies arise due to a range in our view angle to their rotation axes or to a range in their rotation rates (e.g., because that affects the location of the Keplerian corotation radius relative to the sublimation radius) or to some other hidden parameter. After assembling the data we had available, we nevertheless felt it was helpful to sort the stars into the three groups in order to emphasize the shared properties within each group.

Table 1 provides a list of all 23 stars we identified as belonging to these three classes, along with some of their overall defining properties. Spectral types are known for 20 of these stars; all are M dwarfs, with 17 of the 20 having spectral types between M4.0 and M5.5. Most of the Upper Sco K2 sample are $\mathbf{M}$ dwarfs, but the stars in Table 1 are predominantly weighted toward later $\mathrm{M}$ subtypes (this is also reflected in their CMD locations-see Figure 23). Eighteen of the stars in Table 1 have known $\mathrm{H} \alpha$ equivalent widths, all but one of which have equivalent widths appropriate for WTTS $(-3.0 \AA<$ EqW $<-16 \AA)$. Thirteen of the stars in Table 1 have measured Li $6708 \AA$ equivalent widths, all of which are $\sim 0.5 \AA$, also as appropriate for WTTS mid-to-late M dwarfs (the spectral types for two stars and the lithium data for six stars are from our own HIRES spectra, as described in the Appendix).

Eleven of $23(48 \%)$ of the stars in Table 1 have two LombScargle periods (i.e., are apparent binaries), which seems high. From the full sample of $K 2 \mathrm{M}$ dwarf WTTs with $2<P<8$ days ( $\sim 280$ stars), only $16 \%$ are apparent binaries by the same measure. However, for the $\sim 140 K 2 \mathrm{M}$ dwarfs with $P<1$ day, $38 \%$ are apparent binaries. Thus, the stars in Table 1 may have a high binary fraction simply because the rapidly rotating low-mass stars in Upper Sco are often binaries. This is consistent with the conclusion from Stauffer et al. (2016) that M dwarfs in the Pleiades with two Lomb-Scargle peaks are both more rapidly rotating and the two periods are closer together than would have been true if their periods were drawn at random from the single star population.

Figure 1 shows phased light curves for two examples of each of the three classes; phased light curves for all members of the scallop shell and persistent flux-dip classes are provided in the Appendix, along with a description of the processing we have done to the original light curves. The class definitions are as follows.

1. Scallop shells_-phased light curves that look like the rim of a scallop shell. They have many wave-shaped undulations in their phased light curves. Usually the shape remains nearly constant for the entire $K 2$ campaign, but in some cases it changes over time. Peak-to trough amplitudes from a few percent to more than $10 \%$; periods always less than 0.65 days. Eleven of our Upper Sco stars fall in this category.

2. Persistent flux-dip class-discrete triangularly shaped flux dips, generally covering less than 0.15 in phase (for each of up to 4 dips). The flux dips are often more or less stable in depth over the $K 2$ campaign, with dip depths generally a few percent. Outside of the dips, the phased light curves are either nearly flat or consistent with sinusoidal variation due to cool star spots. Periods range from 0.5 to 1.8 days. A half dozen rapidly rotating, late $\mathrm{M}$ dwarf members of the Pleiades with this light-curve signature were identified from $K 2$ Campaign 4 data (Rebull et al. 2016, Section 3.6; A. Collier Cameron et al. 2017, in preparation). Eight of our Upper Sco stars fall in this category.

3. Transient, narrow dip class-more or less triangularly shaped flux dips, narrow in phase, but always variable in depth, on both short and long timescales, over the 78-day $K 2$ campaign. Only one prominent dip is present in the phased light curve. Dip depths are generally a few percent, but up to $20 \%$ for EPIC 205483258 (RIK-210). All four stars also show a spotted-star waveform, whose period is identical to the dip period within our uncertainties. Periods ranging from 1 to 5.5 days.

In the next sections, we discuss specific examples of these categories.

\section{Stars with Scallop-shell Phased Light Curves}

As illustrated in the top row of Figure 1, these stars show complex phased light curves, with many local maxima and minima. For half of these stars, their waveform is relatively stable for the entire 78-day $K 2$ campaign. However, for 5 of the 11 stars we put in this category, there is distinct evolution in the waveform within the campaign. Because we believe the nature of this evolution provides important clues as to the physical mechanism responsible for creating this light-curve morphology, we discuss in detail these five stars and their light-curve evolution during the campaign. First, however, we illustrate the class by describing the light curve of one of the six members whose phased light-curve shape does not change appreciably over the duration of the $K 2$ campaign. 
Table 1

List of Upper Sco Stars with Unusual Periodic Light Curves

\begin{tabular}{|c|c|c|c|c|c|c|c|c|c|c|c|c|c|}
\hline$\overline{\text { EPIC ID }}$ & $\mathrm{Name}^{\mathrm{a}}$ & $\begin{array}{l}\text { R.A. } \\
\text { (deg) }\end{array}$ & $\begin{array}{l}\text { Decl. } \\
\text { (deg) }\end{array}$ & $\begin{array}{c}K_{s} \\
(\mathrm{mag})\end{array}$ & $\begin{array}{c}V-K_{s}{ }^{b} \\
(\mathrm{mag})\end{array}$ & $\begin{array}{c}{[W 1]-[\mathrm{W} 3]} \\
\text { (mag) }\end{array}$ & $\begin{array}{l}\lambda_{\max }{ }^{\mathrm{c}} \\
(\mu \mathrm{m})\end{array}$ & $\mathrm{SpT}^{\mathrm{d}}$ & $\begin{array}{c}\mathrm{H} \alpha \underset{(\AA)}{\mathrm{EqW}} \\
(\AA)\end{array}$ & $\begin{array}{c}\mathrm{Li} \underset{(\AA)}{\mathrm{EqW}} \\
(\AA)\end{array}$ & $\begin{array}{l}\mathrm{P}^{\mathrm{e}} \\
\text { (days) }\end{array}$ & $\begin{array}{l}\mathrm{P}^{\mathrm{e}} \\
\text { (days) }\end{array}$ & Class $^{\mathrm{f}}$ \\
\hline 204918279 & RIK-21 & 239.1046 & -20.2711 & 9.86 & $6.90 \mathrm{~d}$ & 0.38 & 12 & M5.0 & -10.2 & 0.61 & 0.4594 & $0.4665^{*}$ & 1 \\
\hline 204066898 & UScoCTIO 80A & 239.6509 & -23.8005 & 10.19 & 5.59 & 0.27 & 12 & M3 & -9.9 & $\cdots$ & $0.3956^{*}$ & 0.5386 & 1 \\
\hline 203462615 & RIK-42 & 239.9086 & -26.0565 & 10.25 & 6.13 & 0.47 & 12 & M5.5 & -16.2 & 0.81 & $0.5201^{*}$ & 0.4421 & 1 \\
\hline 204897050 & UScoCTIO 56 & 240.4208 & -20.3689 & 10.86 & $7.16 \mathrm{~d}$ & 0.42 & 12 & M5 & $\ldots$ & $\cdots$ & 0.2639 & $\ldots$ & 1 \\
\hline 202724025 & RIK-90 & 242.2373 & -28.5993 & 9.63 & 5.78 & 0.33 & 12 & M4.5 & -10.2 & 0.21 & $0.2595^{*}$ & 0.2795 & 1 \\
\hline 204117263 & LHJ-65 & 242.7589 & -23.5973 & 10.95 & 6.42 & 0.35 & 12 & M5 & -11.6 & $\cdots$ & 0.6423 & $\ldots$ & 1 \\
\hline 204367193 & LHJ-77 & 242.9766 & -22.6137 & 13.30 & 8.33 & $\cdots$ & 4.6 & M6.3 & -10.3 & $\cdots$ & 0.4835 & $\cdots$ & 1 \\
\hline 203534383 & $\cdots$ & 243.5125 & -25.8148 & 11.71 & 7.73 & 0.73 & 12 & M4: & -9.5 & $\cdots$ & $0.2784^{*}$ & 0.3234 & 1 \\
\hline 205110559 & $\cdots$ & 243.8322 & -19.3520 & 10.46 & 7.74 & 0.61 & 12 & $\cdots$ & $\cdots$ & $\cdots$ & 0.4031 & $\cdots$ & 1 \\
\hline 203050730 & RIK-246 & 247.7741 & -27.4295 & 10.53 & 5.87 & 0.53 & 12 & M4.5 & -9.1 & 0.38 & $0.4865^{*}$ & 0.7740 & 1 \\
\hline 203185083 & RIK-253 & 248.6464 & -26.9675 & 10.48 & 6.62 & 0.18 & 12 & M4.5 & -27.1 & 0.54 & 0.4400 & $\cdots$ & 1 \\
\hline 204364515 & $\ldots$ & 240.3398 & -22.6240 & 10.05 & 6.84 & 0.34 & 24 & M4 & -8.4 & 0.55 & 3.0863 & $1.456^{*}$ & 2 \\
\hline 203849738 & RIK-100 & 242.4703 & -24.6982 & 10.93 & $6.69 \mathrm{~d}$ & 0.37 & 12 & M5.5 & -15.1 & 0.40 & 0.6190 & $\cdots$ & 2 \\
\hline 203692610 & $\cdots$ & 242.6318 & -25.2671 & 11.60 & 5.92 & 0.28 & 12 & M4 & -3.0 & 0.54 & 1.821 & $\cdots$ & 2 \\
\hline 205374937 & $\ldots$ & 242.8255 & -17.9580 & 9.33 & 6.35 & 0.34 & 24 & M4 & -4.8 & 0.55 & $0.6345^{*}$ & 0.5436 & 2 \\
\hline 202873945 & $\cdots$ & 245.4164 & -28.0518 & 11.24 & 8.21 & 0.75 & 12 & $\cdots$ & $\cdots$ & $\cdots$ & 0.6258 & $\cdots$ & 2 \\
\hline 204296148 & $\cdots$ & 246.2158 & -22.8952 & 10.99 & $8.06 \mathrm{k}$ & 0.10 & 12 & $\ldots$ & $\cdots$ & $\cdots$ & $0.5314^{*}$ & 0.4717 & 2 \\
\hline 203962559 & AOC 64 & 246.7103 & -24.2312 & 10.80 & $8.83 \mathrm{~d}$ & $\cdots$ & 8 & M4 & -6.0 & $\cdots$ & 1.5402 & $\cdots$ & 2 \\
\hline 203927435 & WMR 2-23 & 247.1794 & -24.3812 & 10.14 & $9.11 \mathrm{~d}$ & 0.13 & 12 & M4.5 & -6.8 & 0.76 & $0.4820^{*}$ & 0.4162 & 2 \\
\hline 205024957 & $\cdots$ & 242.5459 & -19.7678 & 11.38 & 6.53 & 0.46 & $24^{+}$ & M5 & -4.4 & 0.56 & 1.6656 & $\ldots$ & 3 \\
\hline 205046529 & $\cdots$ & 242.6099 & -19.6642 & 10.40 & $6.52 \mathrm{k}$ & 0.35 & 24 & M4 & -4.4 & 0.54 & 2.5619 & $1.8358^{*}$ & 3 \\
\hline 204143627 & LHJ-120 & 243.9969 & -23.4934 & 11.31 & 6.29 & 0.13 & 12 & M5 & $\cdots$ & $\cdots$ & 1.125 & $\cdots$ & 3 \\
\hline 205483258 & RIK-210 & 245.8523 & -17.2909 & 9.65 & 5.04 & 0.37 & 12 & M2.5 & -5.3 & 0.59 & 5.667 & $\cdots$ & 3 \\
\hline
\end{tabular}

Notes.

${ }^{a}$ Sources of the names are: RIK: Rizzuto et al. (2015); UScoCTIO: Ardila et al. (2000); LHJ: Lodieu et al. (2007); AOC: Alves de Oliveira \& Casali (2008); WMR: Wilking et al. (2005).

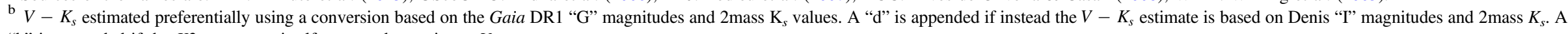
"k" is appended if the $K 2$ count rate itself was used to estimate $V$.

${ }^{\mathrm{c}}$ Longest wavelength at which there is a secure detection, in $\mu \mathrm{m}$. A plus sign indicates if there is a plausible, small IR excess.

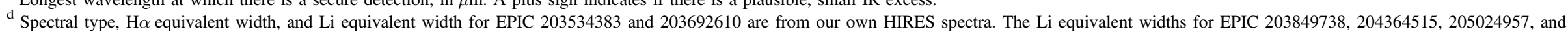
205046529 are also from our own HIRES spectra. See the discussion in the Appendix.

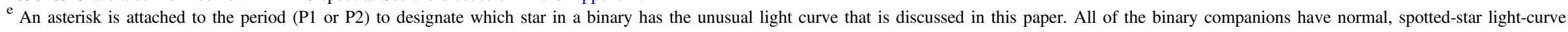
morphologies.

${ }^{\mathrm{f}}$ Light-curve classes: 1 = scallop shell; 2 = persistent short-duration flux dip; 3 = transient short-duration flux dip. 

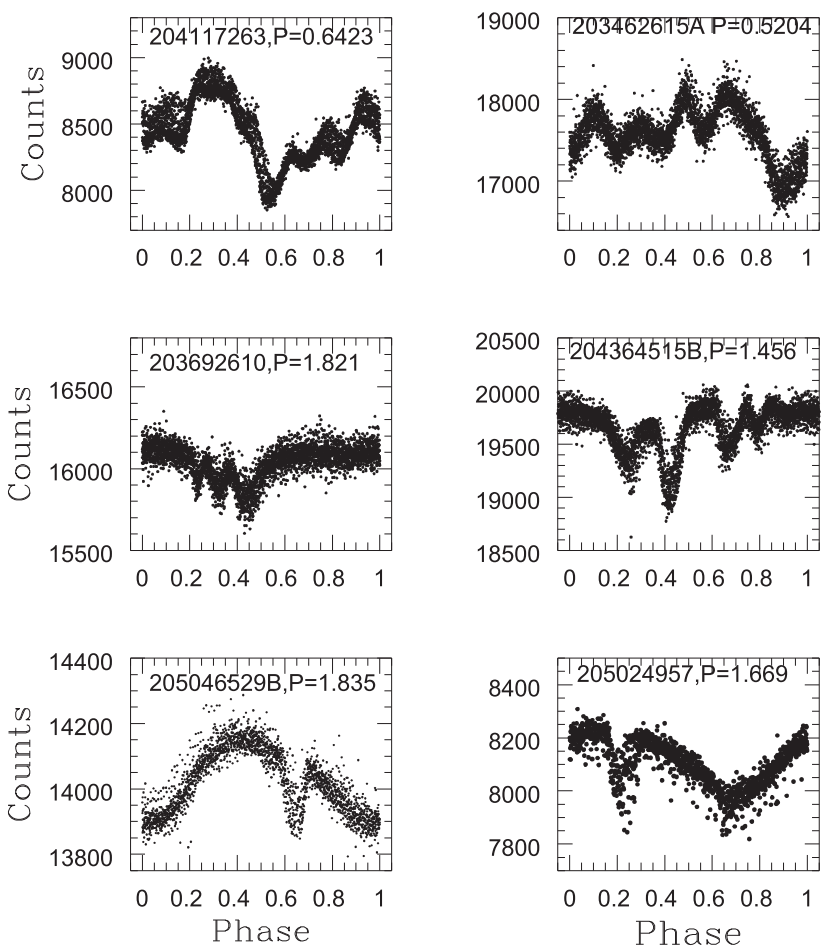

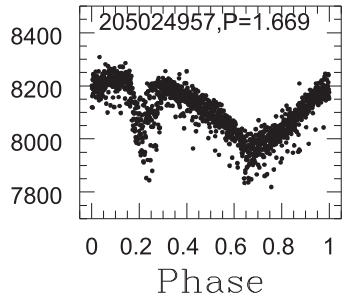

Scallop-shell

Persistent Flux Dip

Transient Flux Dip

Figure 1. Phased light curves for exemplary members of the "scallop-shell" light-curve class (top row), the persistent, short-duration flux-dip class (middle row), and the transient short-duration flux-dip class (bottom row).

\subsection{EPIC $204066898 a$}

EPIC 204066898 (a.k.a. UScoCTIO 80A ${ }^{11}$; Kraus \& Hillenbrand $2009=\mathrm{KH} 2009$; Kraus \& Hillenbrand $2012=\mathrm{KH} 2012$ ) is an M3 member of Upper Sco with no apparent IR excess. It has been identified as a wide binary, with separation about $13^{\prime \prime}$ and $\Delta K_{s}=1.9 \mathrm{mag}$ (Kraus \& Hillenbrand $2009=\mathrm{KH} 2009)$. KH2012 also identified UScoCTIO $80 \mathrm{~A}$ as a close $(0 . \prime 05)$, nearly equal mass binary (see their Table 3). We find two independent peaks in the Lomb-Scargle periodogram with periods of 0.3956 and 0.5386 days. We experimented with 1-pixel and 1.5-pixel apertures for the light-curve extraction in order to assess the contribution of UScoCTIO 80B to the EPIC 204066898 light curve; our conclusion is that the two Lomb-Scargle peaks are both correctly associated with UScoCTIO 80A (UScoCTIO 80B is also EPIC 204066097, for which we derive a period of $0.36 \mathrm{~d})$. We thus assume the two stars associated with the two Lomb-Scargle peaks are also the close binary pair identified in KH2012. The longer period system for EPIC 204066898 (which we designate as the secondary component) has a more or less sinusoidal light curve with about $1 \%$ peak-to-trough amplitude. After removing the signal from this system and phasing the remaining signal to the 0.3956-day period of the primary component, the light-curve shape for this star (Figure 2) is fairly typical of the scallop-shell class, with a full amplitude of about 5\% and multiple peaks and troughs in its phased light curve.

\footnotetext{
${ }^{11}$ KH2009 actually call this star USco 80A, but in keeping with the SIMBAD usage, the more appropriate name is UScoCTIO 80A. The star designated UScoCTIO 80 in Ardila et al. (2000) is UScoCTIO 80B, as explained in the note to Table 9 of $\mathrm{KH} 2009$.
}

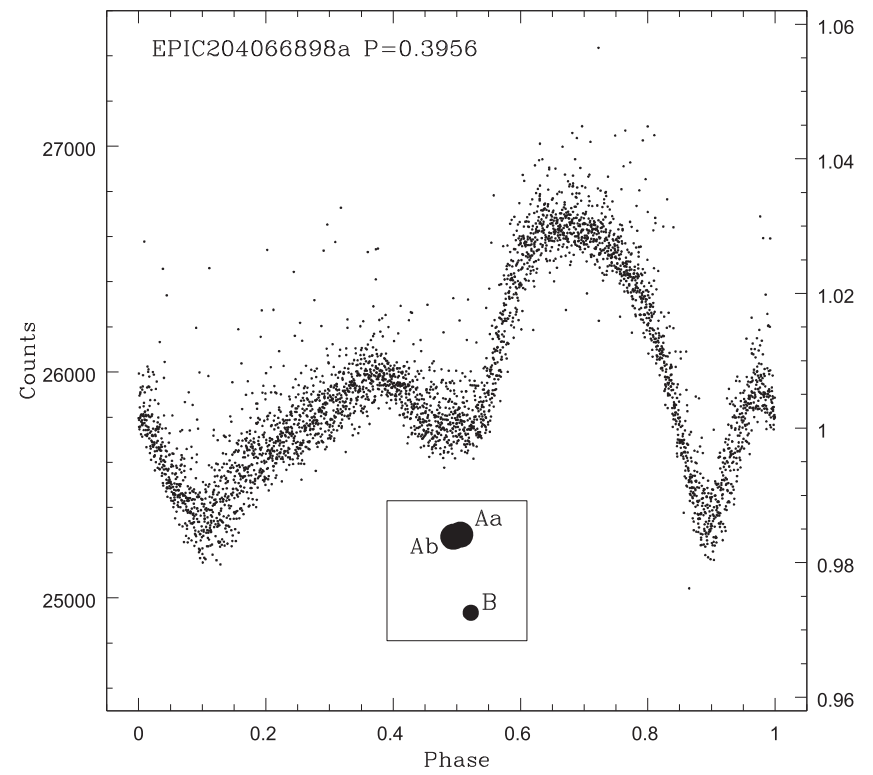

Figure 2. Phased light curve of EPIC 204066898a (UScoCTIO 80Aa). The left-side $y$-axis is in "counts"; the right-side $y$-axis normalizes to the median count rate in order to allow easy determination of amplitudes in terms of percent change in the light-curve flux. The light curve shows no evidence for significant change over the 78 days of the $K 2$ campaign. The phased light curve shows more structure than can be produced by cool spots on the photosphere. The inset is a not-to-scale spatial cartoon of the orientation of the Upper Sco CTIO 80 triple system; the actual separation between "Aa" and "Ab" is 0 ". 05 and between "Aab" and "B" is 13 ". This cartoon does not represent the $K 2$ stamp image for this system nor the aperture we used for the photometry.

\subsection{EPIC 205110559}

There are no published spectra for EPIC 205110559, but its colors suggest it is a mid-to-late $\mathrm{M}$ dwarf. Its light curve (Figure 3 ) is also fairly typical of the scallop-shell class, with a 

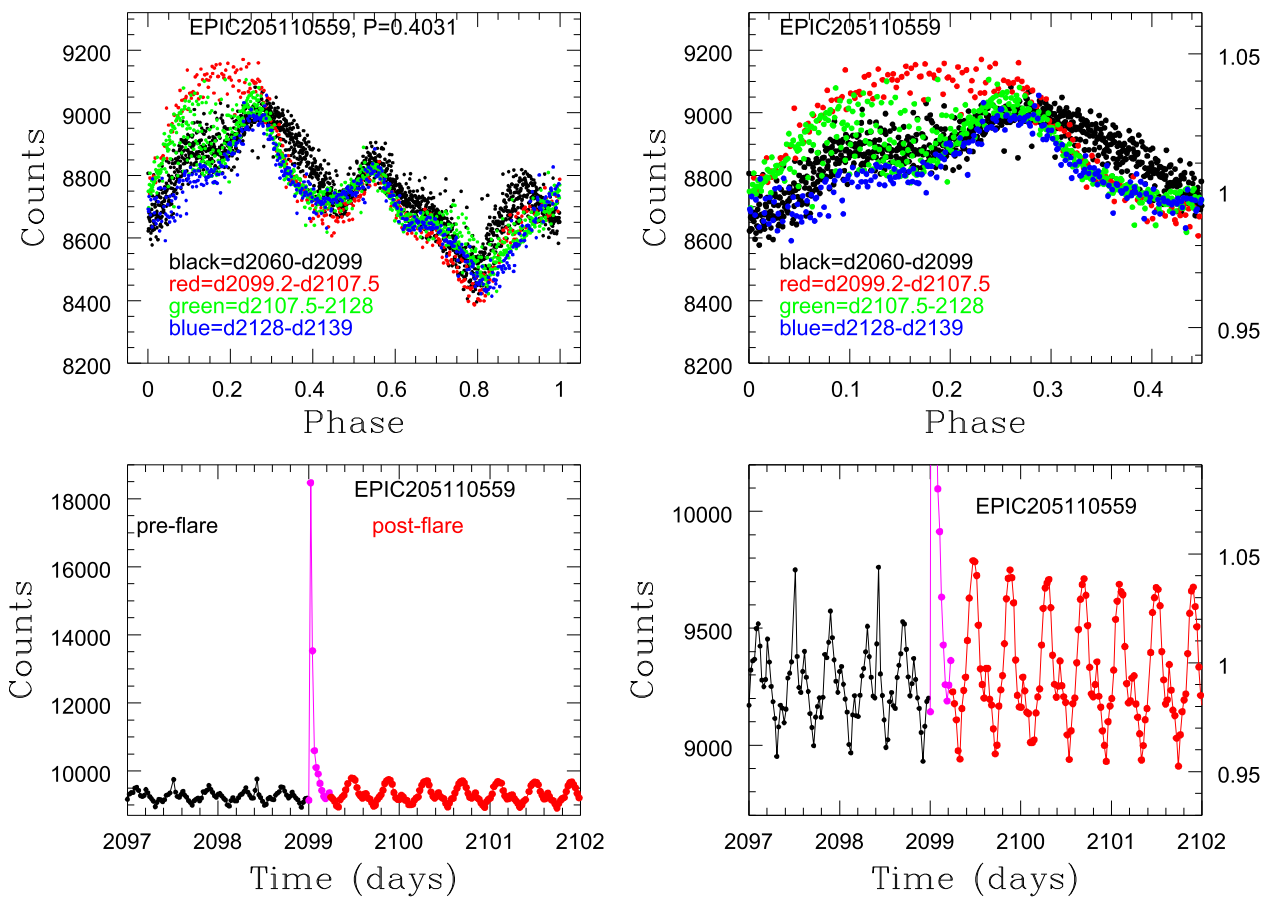

Figure 3. Phased light curve and other plots to illustrate the light-curve evolution of EPIC 205110559. (Top left) Full phased light curve. The different colors correspond to the windows in time noted within the plot. (Top right) Expanded view of just the first half of the phased light curve. (Bottom left) Strong flare occurred at day 2099 (by far the strongest for this star during the $K 2$ campaign). (Bottom right) Expanded view of the light curve around the time of the flare, showing that the periodic waveform changed following the flare. For the top-right and bottom-right plots, $y$-axis labels for both counts and counts normalized to the median value are provided.

full amplitude of about $5 \%$ and multiple peaks and troughs in its phased light curve. For phase $0.45-1.0$, the waveform is relatively stable over the duration of the campaign. However, as emphasized in the second panel of Figure 3, the waveform shows considerable evolution over the campaign for the phase range $0-0.45$. The primary shift in waveform shape appears to occur almost exactly at the boundary between the first window (day 2060-2099) and the second window (day 2099.2-2107.5). ${ }^{12}$ The third panel of Figure 3 shows an expanded view of the time frame when the waveform changed state, which reveals that a strong flare occurred at day 2099. This is by far the strongest flare for this star during the $K 2$ campaign and it occurs at exactly the point that the waveform made its state transition. This latter point is illustrated in the last panel in Figure 3. The "excess" flux at phase $\sim 0.15$ gradually declines, returning to approximately the pre-flare level by day $\sim 2128$. We conclude that there is very likely a physical link between the occurrence of the flare and the change in morphology of the phased light curve.

\subsection{EPIC 204918279B}

The $K 2$ light curve for EPIC 204918279 has a beating appearance and the Lomb-Scargle periodogram shows two strong peaks at very similar periods, 0.4594 and 0.4665 days, which we interpret as indicating that it is a binary with the two components having nearly the same period.

After separating the variability at those two periods, we find that the star with $P=0.4665$ days has a light-curve shape that places it in the scallop-shell category at least for the first $\sim 60$ days

\footnotetext{
12 The times listed here and throughout the remainder of the paper are in days since 2009 January 1, or as JD-2454833. Kepler was launched on 2009 March 7.
}

of the $K 2$ campaign, as shown in the first panel of Figure 4. The phased light curve changed abruptly in shape at day $\sim 2123.5$, as illustrated in the second and third panels of Figure 4. The state transition of the light-curve shape again appears to have been triggered by a flare; see the fourth panel of Figure 4. In this case, this is a much weaker flare than for EPIC 205110559. ${ }^{13}$ Nevertheless, this was the strongest flare seen in the $K 2$ light curve for EPIC 204918279, at least during the last 60 days of the campaign. Prior to and after the flare, the light -curve shape appeared quite stable during the $K 2$ campaign.

\subsection{EPIC 203185083}

EPIC 203185083 is an M4.5 YSO Upper Sco member with unusually strong $\mathrm{H} \alpha$ emission (equivalent width $=-27 \AA$ ) for a WTTS of that spectral type (Rizzuto et al. 2015). However, it appears to have no IR excess, so we consider it to be a WTTS. The Lomb-Scargle periodogram indicates a period of 0.4400 days.

After phasing the light curve to the 0.44-day period, we find a waveform shape that is not plausibly explained by cool spots and that seems to evolve over the duration of the $K 2$ campaign. After some investigation, we find the waveform evolution basically occurs at two rapid transitions on day 2093 and day 2102, as shown in the left panel of Figure 5. The first state transition of the light-curve shape again appears to have been triggered by a flare (right panel of Figure 5), though again a much weaker flare than for EPIC 205110559. Nevertheless, this was the strongest flare seen in the $K 2$ light curve for EPIC

\footnotetext{
${ }^{13}$ Because this is a binary, we cannot know for certain which of the two stars was the site of the flare. However, because of the close coincidence between the flare and the change in light-curve waveform for the star with $P=0.4665$ days, we believe it is highly probable the flare originated on that star.
} 

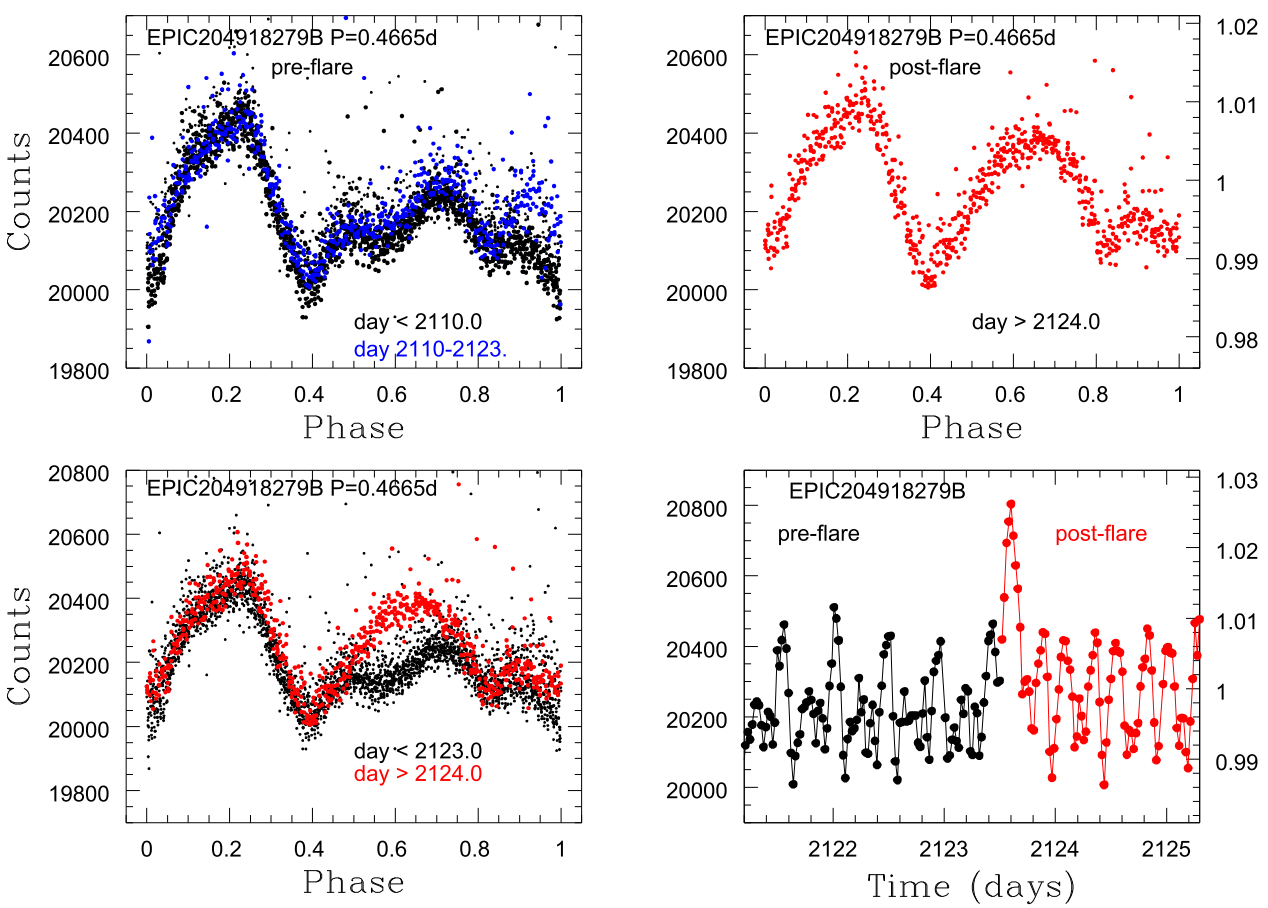

Figure 4. Phased light curve and other plots to illustrate the light-curve evolution of EPIC 204918279B. (Top left) Phased light curve for $d<2123$. (Top right) Phased light curve for $d>2124$. (Bottom left) Superposition of the two previous panels, showing that the change in waveform was restricted to the phase range $0.45-0.7$. (Bottom right) Expanded view of the light curve around the time of the phase transition, showing the presence of a small flare when the change occurred.
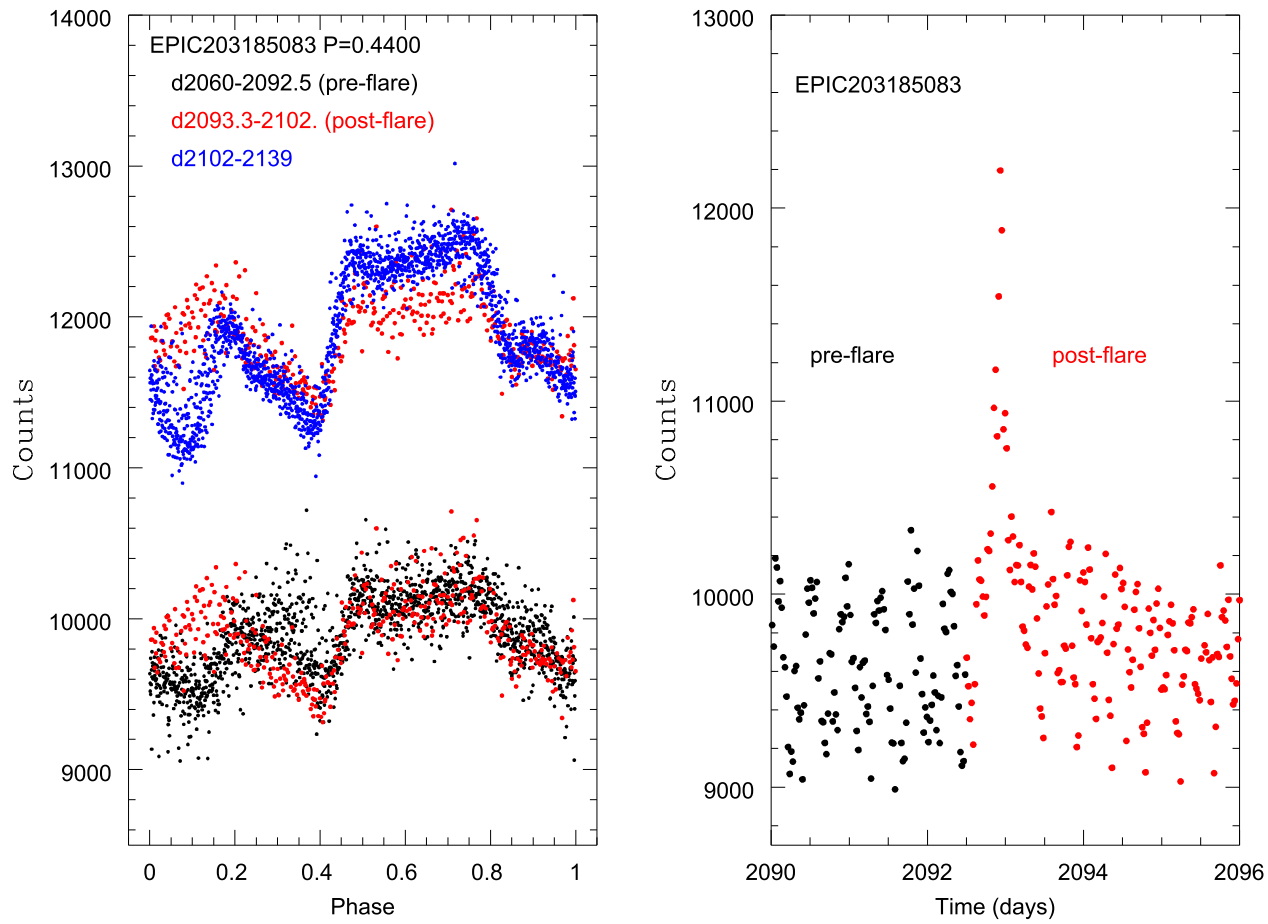

Figure 5. Plots illustrating the light-curve evolution of EPIC 203185083. (Left) Phased light curve for three time intervals: blue (day 2060-2092.5), green (day 2093.3-2102), and red (day 2102-2139). There is a transition in waveform shape at day 2093, and another transition in shape at day 2102. The light curves at the top of the plot are offset in y by 2000 counts for clarity. (Right) Expanded view of the light curve near the first state transition in the phased waveform, showing the presence of a small flare when the change occurred.

203185083. The second state transition appears to happen without any obvious trigger as seen from our vantage point. Finally, for both EPIC 203185083 and EPIC 204918279, the flares at the state transition seem unusual in that they have nearly symmetric shape (rather than a rapid rise and slow decay as true for most flares and for the flare on EPIC 205110559). 


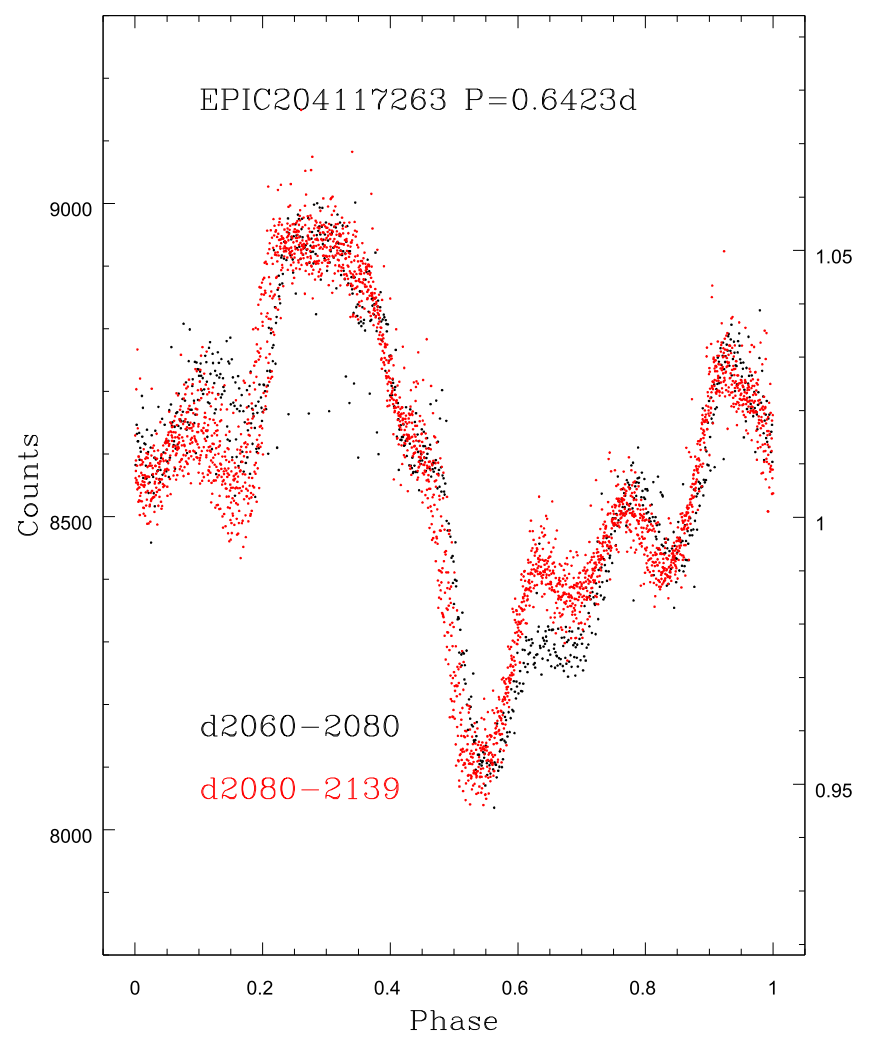

Figure 6. Phased light curve of EPIC 204117263, illustrating the abrupt change in shape at day 2080. Black points correspond to the time window for days 2060-2080; red points correspond to data after day 2080. The largest change in waveform shape is near phase $=0.65$, but there is also a small change near phase 0.15 . Otherwise, the shape of the phased light curve was very stable over the entire $K 2$ campaign.

\subsection{EPIC 204117263}

The Lomb-Scargle periodogram for EPIC 204117263 shows a single strong peak at $P=0.6423$ days; when phased to that period, the light curve has a scallop-shell appearance for the entire campaign. There was, however, a small, abrupt change in the phased light-curve shape at day 2080.0, as illustrated in Figure 6. In this case, the state transition in the light-curve shape does not appear to be associated with a flare (at least on the side of the star that is facing us).

\subsection{EPIC 204897050}

The Lomb-Scargle periodogram for EPIC 204897050 shows a dominant strong peak at a very short period of $P=0.2639$ days; when phased to that period, the light curve has a scallop-shell appearance for the entire campaign. As illustrated in Figure 7, there is a small portion of the phased light curve around phase $=0.6$ where the waveform shape gradually changes throughout the length of the campaign. This could be consistent with the other three stars discussed above, if there was a trigger event just prior to the beginning of Campaign 2; no significant flares are seen during the time period of our light curve.

\subsection{Shared Characteristics of the Stars with Scallop-shell Light Curves}

The stars having scallop-shell light curves have many shared characteristics, which presumably provide clues to the physical

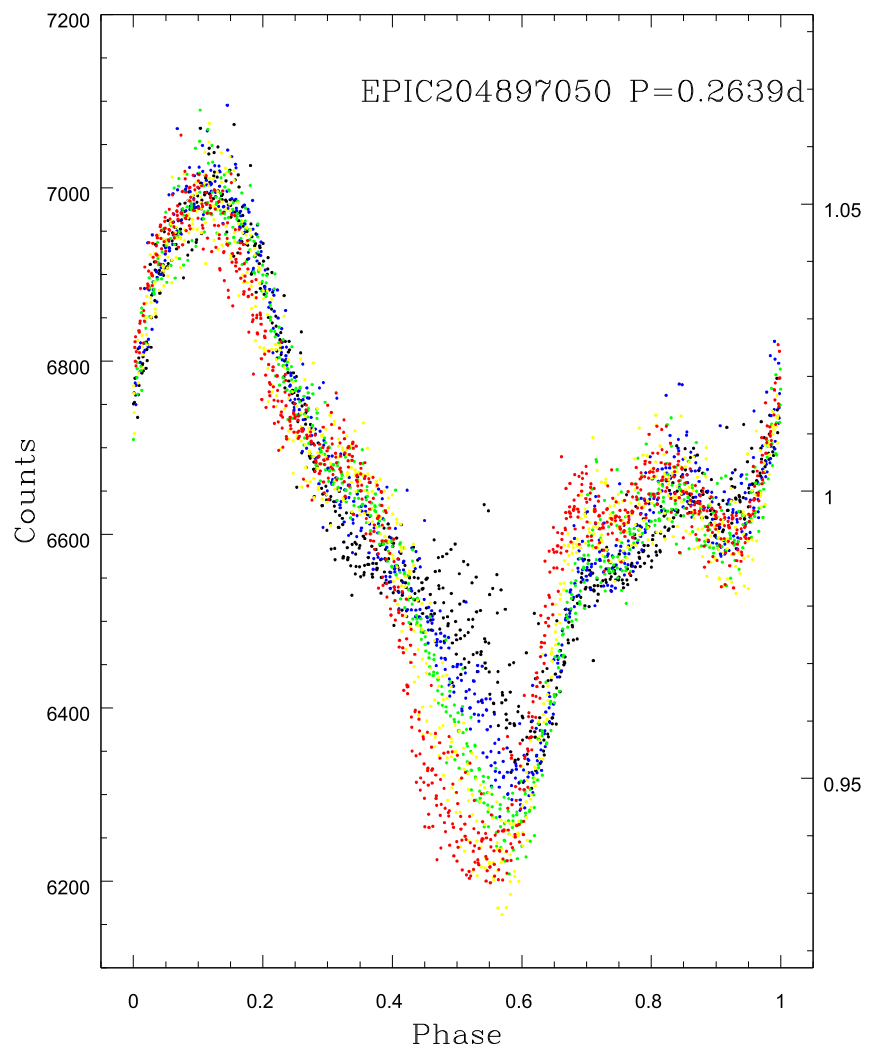

Figure 7. Phased light curve of EPIC 204897050, illustrating the gradual change in the shape of the phased light curve near phase $=0.60$. Otherwise, the shape of the phased light curve was stable over the entire $K 2$ campaign. Colors (black, blue, green, yellow, red, in order) correspond to approximately 15 day long windows. The flux minimum near phase 0.5 became deeper and broader as the campaign progressed.

process driving their unusual light-curve morphology. Below we collect the most salient of those shared characteristics.

1. All have periods less than 0.65 days. We do not know that this is, in fact, the stellar rotation period, but we will assume that is the case. With that assumption, their periods place them among the most rapidly rotating $\mathrm{M}$ dwarfs in Upper Sco (see also Section 7).

2. All have mid-to-late M spectral types (M4 to M6.5).

3. They have full amplitudes for their $K 2$ light curves of $2 \%-10 \%$.

4. Six of 11 of them show relatively stable phased lightcurve shapes over the entire 78 day $K 2$ campaign.

5. Five of them show evolving waveforms. In all but one case, there is an abrupt transition from one waveform to another with the switch happening in generally less than a day. In three cases, the phase change takes place when a strong flare occurs. In four of the five cases, the change in shape only significantly affects $20 \%-30 \%$ of the waveform, with the remaining waveform staying essentially constant in shape. For EPIC 205110559, the fractional flux change between states at phase $=0.1$ is of order $4 \%$ (Figure 3, second panel). Any physical mechanism to explain this light-curve morphology must be able to accommodate events of this size. For all three events with triggering flares, the affected portion of the light curve brightened after the flare.

6. The number of distinct peaks (or troughs) in their phased light curves ranges from 3 to 6 . 
7. There are 83 WTTs in our sample with $P<0.65$ days and $\left(V-K_{s}\right)>5$. The fraction that have scallop-shell light-curve morphologies for that period range is therefore $13 \%$.

8. We have also examined this WTT control group for obvious flares. Based on a simple visual check of the detrended light curves of our Table 1 stars and our WTT control group, we find no obvious difference in either the frequency or strength of flares.

\section{Stars with Short-duration, Persistent Flux Dips}

There are eight stars in Table 1 in this category. The phased light curves of these stars are dominated by shallow triangularly shaped flux dips, superposed either on a flat "continuum" or on a waveform consistent with that due to rotational modulation of cool spots at the same period as the flux dips. Four of these stars show little or no evolution in their phased light-curve morphology over the time frame of the $K 2$ campaign; we describe one of these four stars (EPIC 205374937) in detail. The other four members of the class show demonstrable evolution in their light-curve morphology over the $K 2$ campaign, and we discuss each case individually in order to illustrate the nature of this evolution.

\subsection{EPIC 205374937}

Preibisch et al. (2001) first identified EPIC 205374937 as a low-mass member of Upper Sco. Carpenter et al. (2009) and Luhman \& Mamajek (2012) both reported no IR excess. Using Gemini North adaptive optics (AO) imaging, Lafreniere et al. (2014) identified EPIC 205374937 as a nearly equal mass binary with a 0 ". 1 separation. The $K 2$ light curve shows two approximately equal strength close peaks, corresponding to periods of 0.6345 and 0.5436 days. We assume the two nearly equal mass components seen with the $\mathrm{AO}$ imaging give rise to these two periodogram peaks; we somewhat arbitrarily assign the the larger periodogram peak as the A component. The phased light curve for the A component is shown in Figure 8.

We interpret the phased light curve for EPIC 205374937A as the superposition of a more or less sinusoidal variation due to star spots and two (or possibly three) short-duration flux dips. The two most prominent flux dips are centered at phases about 0.2 and 0.6; the full width at zero intensity (FWZI) of the dips are 0.16 and 0.12 in phase (or about $2 \mathrm{hr}$ in time) and their depths are about $3 \%$ of the continuum. There is no suggestion of the dips varying in depth or width over the course of the campaign (that is, the noise level in the phased light curve appears the same inside and outside the dips). The period that phases the dips best also produces the least scatter in the rest of the light curve, indicating that the dip period is essentially the same as the spot period. Two of the other stars in this group (EPIC 204296148 and 203849738) also have both spotted-star waveforms and persistent flux dips where the periods are the same (see Figure 36).

\subsection{EPIC 203692610}

EPIC 203692610 is an essentially anonymous red star with proper motion consistent with Upper Sco membership. Our own Keck HIRES spectra for this star shows it to have a spectral type of about M4, with weak $\mathrm{H} \alpha$ emission (equivalent width $=-3.0 \AA$ ) and strong lithium absorption (equivalent

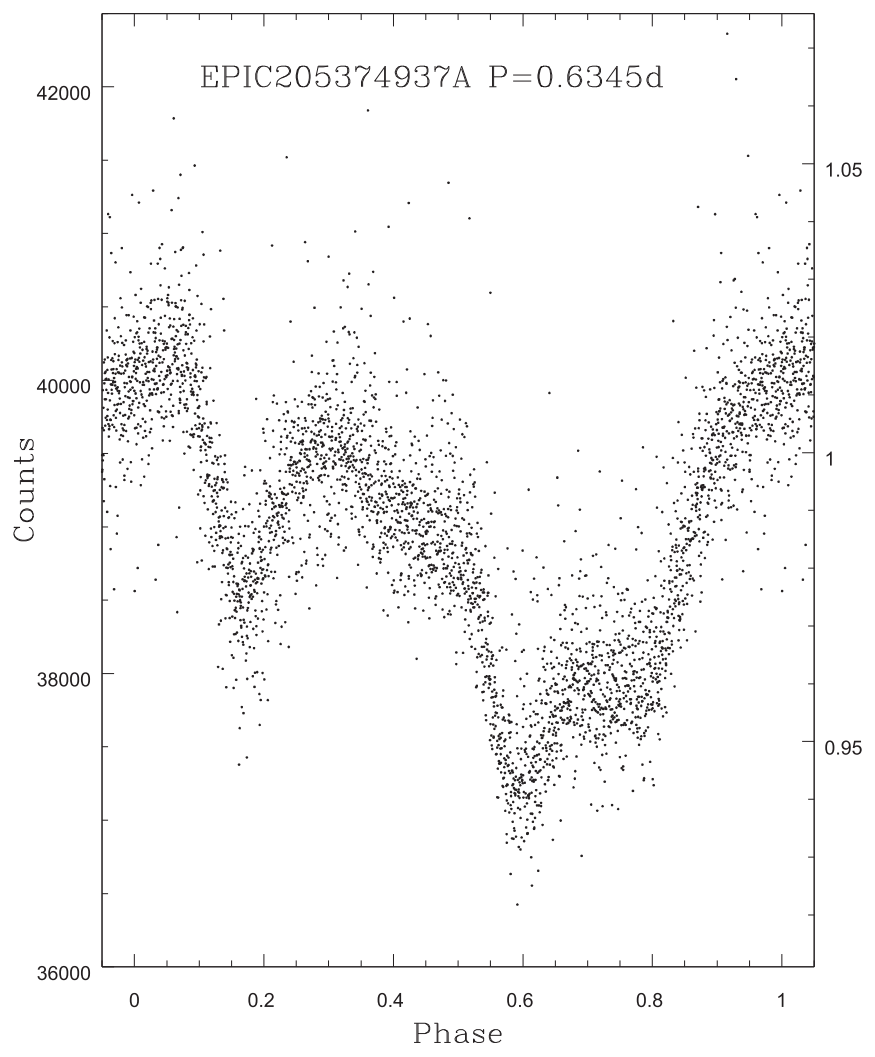

Figure 8. Phased light curve of EPIC 205374937A. The two most prominent features are short-duration triangularly shaped flux dips at phases of about 0.2 and 0.6. These are superposed on a more slowly varying waveform, which is broadly consistent with the approximately sinusoidal shape often produced by non-axisymmetrically distributed star spots.

width $=0.54 \AA$ ), typical of Upper Sco M dwarfs. The LombScargle periodogram for its $K 2$ light curve shows a single strong peak at $P=1.821$ days. ${ }^{14}$ When the light curve is phased to that period, it shows a relatively flat continuum with three sharp triangular-shaped flux dips (Figure 9). The dip depths are about 3\% to 7\%, and the FWZI for each dip is about 0.1 in phase. Close examination of the light curve shows that the dip depths vary in time, with the time dependence being somewhat different for each dip, as illustrated in the top-right panel of Figure 9. The left-most dip is more or less stable in its depth until day 2119, when it essentially disappears. The central dip is more or less stable in depth until day 2131, when it too disappears. The strongest (right-most) dip is quite deep until day 2089; from 2089 to 2131 it is about half as deep (and somewhat shifted in phase), and at day 2131 it becomes significantly weaker again. There are no strong flares in the $K 2$ light curve for this star. However, the strongest flare-like event (or events) occurs at the time of the first state transition of the flux dips at day 2089, as illustrated in the bottom-left panel of Figure 9. There are no obvious flares of any sort at the other two state-transitions. Finally, we note that the dip properties and the general appearance of the phased light curve for EPIC 203692610 are quite similar to the $K 2$ light curve for HHJ 135 , one of the six late $\mathrm{M}$ dwarfs with persistent, short-duration flux dips identified in the Pleiades by Rebull et al. (2016).

\footnotetext{
14 Because there is a brighter star located about $13^{\prime \prime}$ away, we analyzed a light curve derived using a 1.5-pixel aperture for EPIC 203692610, rather than the 2or 3-pixel radius used throughout the rest of the paper.
} 

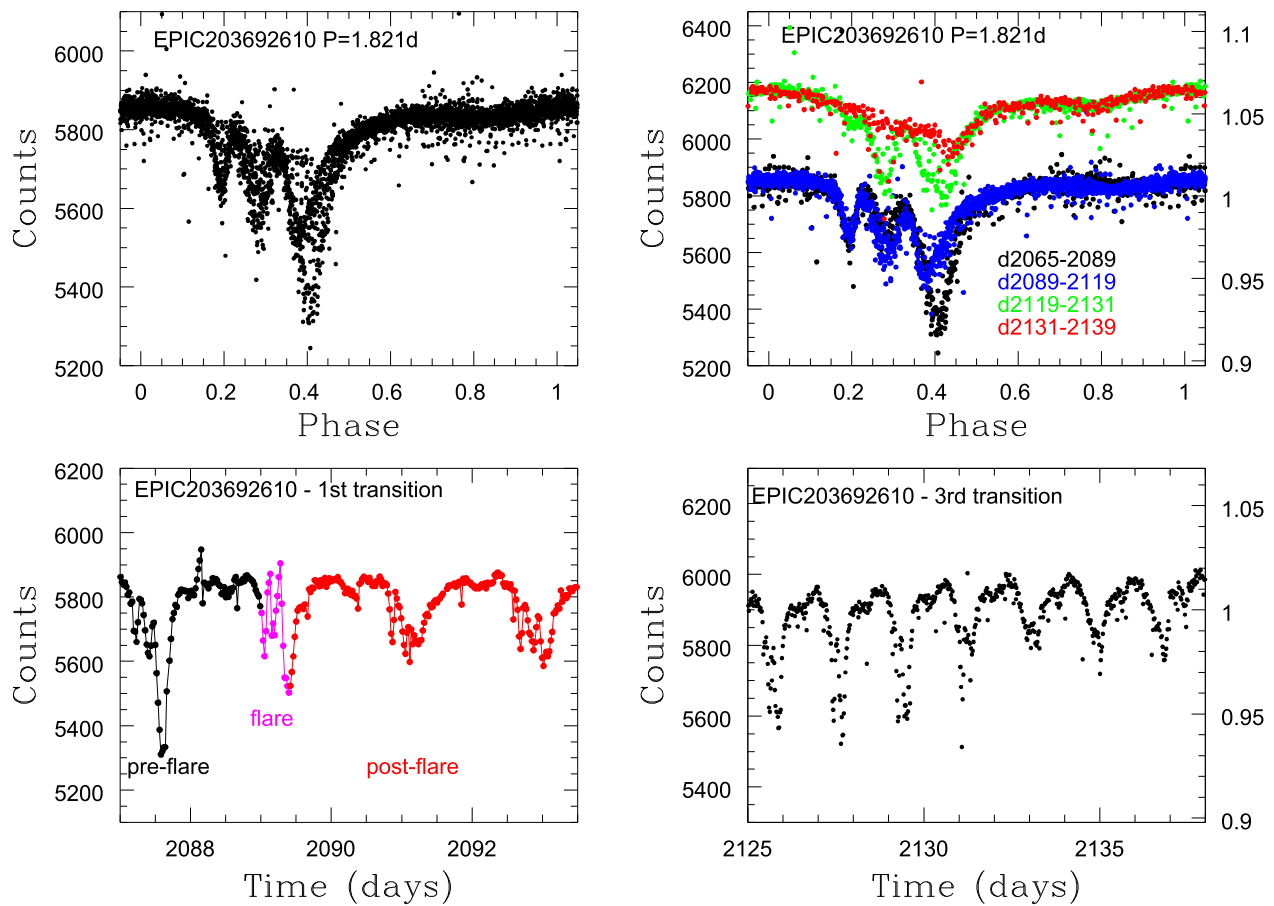

Figure 9. (Top left) Phased light curve of EPIC 203692610 showing data for the full $K 2$ campaign, illustrating the three short-duration flux dips that are present. (Top right) Phased light curve for EPIC 203692610, with the data now broken up into four time windows: black (d2065-2089), blue (d2089-2119), green (d2119-2131), and red (d2131-2139). The green and red curves are shifted vertically by 300 counts. The three flux dips appear to evolve in shape with different timescales, with the main changes appearing to happen in sudden events. (Bottom left) Expanded view of the light curve at the time of the first state transition in the flux dips, showing two possible flares at day 2089 and the much reduced amplitude of the flux dips after that day. (Bottom right) Expanded view of the time period associated with the third state transition of the flux-dip depths (day 2131); no obvious flare or other trigger is present at that time. For the top-right panel, the normalized count-rate values (rhs of the plot) apply to the blue and black points.

\subsection{EPIC 203962559}

While EPIC 203962559 has been identified as a $\rho$ Oph member (Wilking et al. 2005); it appears to have no IR excess and has relatively weak $\mathrm{H} \alpha$ emission, from which we conclude it is a WTTS. The Lomb-Scargle periodogram shows one strong, real peak at $P=1.5402$ days. When phased to that period and examined closely, the light curve appears to undergo a major change in shape at about day 2106. This is illustrated in Figure 10. Prior to that day, there were four welldefined flux dips in the light curve; after that day, only two flux dips remain, and one of those is significantly narrower and less deep. We are unable to determine if there was a flare at day $\sim 2106$ because that date also is when Mars transited the $K 2$ FOV (near the position of EPIC 203962559), corrupting its light curve for $\sim 10 \mathrm{hr}$. We can think of no possible way for the Mars transit to induce systematic flux errors in a specific phase range in the extracted light curve for this star, so we assume this was a bizarre coincidence.

\subsection{EPIC 203927435A}

EPIC 203927435 has also been identified as a $\rho$ Oph member (Wilking et al. 2005); it too appears to have no IR excess and relatively weak $\mathrm{H} \alpha$ emission (equivalent width -6.8 $\AA$ ). The Lomb-Scargle periodogram shows two resolved peaks, with periods of 0.4817 and 0.4162 days. After removing the signal from the star with $P=0.4162$ days (which we refer to as the B component), we find that the phased light curve for the A component shows a waveform that is not explainable by cold spots, but that also shows some evolution during the $K 2$ campaign. The first panel of Figure 11 shows the phased light curve for the first 63 days of the campaign; the light curve for the last 13 days of the campaign is shown in the second panel of Figure 11. A very strong flare occurred at the transition point - third panel of Figure 11-on day 2123.3; this was by far the strongest flare for this star during the $K 2$ campaign. Prior to that day, there were four well-defined flux dips in the light curve; after the flare, only one (weaker) dip remains. In this case, the transition between states took place over about 3 days. During the middle of that transition period, a second weaker flare-like event occurred; see the last panel of Figure 11. That event, however, has a symmetrical shape (as was the case for the triggering events for EPIC 204918279 and EPIC 203185083).

\subsection{EPIC 204364515B}

Bouy et al. (2006) showed that EPIC 204364515 is a possible resolved triple system, consisting of a close, equal brightness pair with a 0 !! 15 separation and a fainter possible companion at a separation of about $1^{\prime \prime}$ (with its $\sim 4^{\prime \prime}$ pixels, the $K 2$ light curve includes light from all three stars). The LombScargle periodogram for EPIC 204364515 shows two dominant periods of 3.0863 and 1.456 days. After removal of the light from the star with $P=3.086$ days, the phased light curve for the star with $P=1.456$ days shows four well-defined flux dips. Figure 12 shows the phased light curve, where we have split the data into two windows of time (black for day 2060-2105; red for day 2105-2139). The two strongest flux dips appear to be approximately stable in position, shape, and depth. The flux dip at phase $\sim 0.2$, however, appears to shift in phase by about 0.03 in phase (10 degrees in azimuth) between the two 


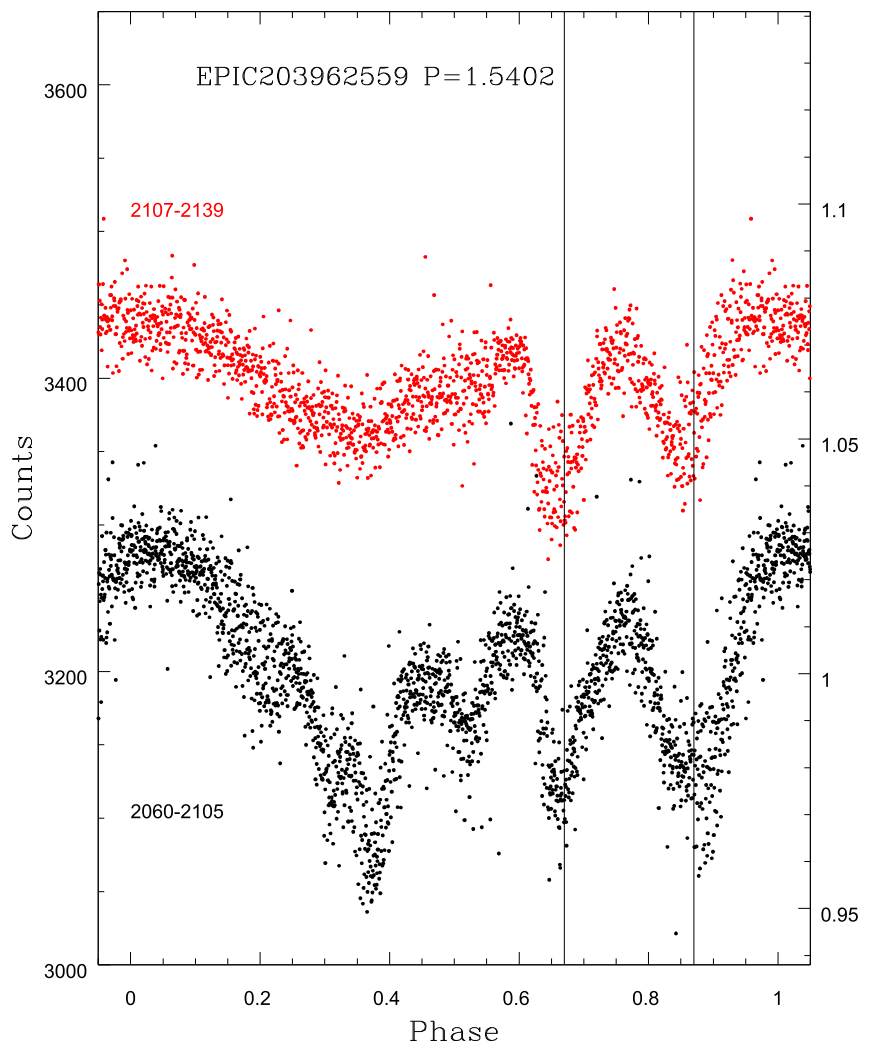

Figure 10. Phased light curve of EPIC 203962559, illustrating the abrupt change in shape at day 2106. The largest change in waveform shape is near phase $=0.3$, but the dip at phase $\sim 0.5$ essentially disappears and the dip at phase $\sim 0.85$ weakens and possibly shifts somewhat in phase. The second time interval data have been offset by 200 counts in the $y$-axis in order to more clearly show the differing light-curve shapes. The rhs $y$-axis normalized count labels are derived for the first time interval (black points).

windows. The fourth flux dip at phase $\sim 0.75$ appears to become considerably deeper in the second time window.

\subsection{Shared Characteristics of the Stars with Persistent Flux Dips}

We measured widths and depths for all the prominent flux dips in these eight stars; see Table 2 . The widths we provide are full-width, zero-intensity values, FWZI, in units of fraction of the period-meaning that they are intended to indicate the entire duration of the event (rather than, for example, a FWHM). Most of the dips have FWZI of 0.1-0.15 in phase; for a typical star with $P=0.6$ days, a FWZI of 0.12 corresponds to about $1.7 \mathrm{hr}$. The depths are the difference in counts between a nearby "continuum" level and the bottom of the dip relative to the continuum level, expressed as a percent. Most of the dips have depths of $1 \%-3.5 \%$, with the strongest being $7 \%$ (the deepest dip for EPIC 203692610).

Additional shared characteristics of these stars include the following.

1. All eight of these stars have $P<2$ days. Five of them have $P<0.65$ days. In three cases, the period derived for the flux dips agrees with the period inferred from a spotted-star waveform, confirming that the dip period is also the stellar rotation period. For the other five, we believe it is likely that the dip period is the rotation period, but that is not proven.
2. All have mid-to-late $\mathrm{M}$ dwarf spectral types (M4 to M5.5).

3. The deepest flux dips for each star have depths from $2 \%$ to $7 \%$; their widths (FWZI) are generally 0.1 to 0.15 in phase, corresponding to about $1-5 \mathrm{hr}$ duration in time.

4. They have two to four discrete flux dips in their phased light curves (at the sensitivity level of the $K 2$ data).

5. For four of these stars, the dip depths appear fairly stable over the duration of the $K 2$ campaign. In three of the other stars, one or more of the flux dips disappears abruptly (or becomes markedly weaker) during the course of the campaign. At least two of the abrupt changes in flux-dip depth occurs immediately following a flare (or flare-like event).

\section{Stars with Short-duration Transient Flux Dips}

There are only four members of this class; we discuss each of them here.

\subsection{EPIC 205046529B}

The Lomb-Scargle periodogram for EPIC 205046529 shows two strong peaks indicating it is a binary, with $P_{1}=2.5619$ days and $P_{2}=1.837$ days. This star appeared as a close pair in the Keck/HIRES guider camera, and we assume the two spatially resolved stars seen with Keck correspond to the two periodogram peaks. After removing the signal from the longer period star and then phasing to 1.837 days, we noted the presence of an apparent flux dip in the light curve for the second star. After further processing, we determined a best-fit period to highlight the flux dip of $P=1.8358$ days. Figure 13 shows the phased light curve; the dominant feature is a spotted-star waveform, superposed on which is a well-defined flux dip centered at phase $\sim 0.6$. The flux dip, however, appears somewhat noisy either due to jitter in the individual dip depths or their timing or both. We believe this fuzziness is primarily due to variations in the depth of the dip both on short and long timescales. Figure 14 shows four 10-day windows in the light curve for EPIC 205046529B. Close examination of those traces shows that the dip depth seems to decrease slowly with time over the $K 2$ campaign, but also varies in depth from cycle to cycle. In order to determine better the nature of the variations in the dip times and depths, we measured the depths and first-moment times of occurrence of all the dips where we could identify that a dip was present. Those times and depths are provided in Table 3. Histograms of the dip depths in two time windows (day 2060-2086 and day 2087-2106) are shown in Figure 15, demonstrating the decreasing dip depth with time. After day 2107, the flux dip was no longer detected with certainty. Figure 16 shows the phased light curves for those same two time windows, also illustrating the decrease in dip strength as the $K 2$ campaign progressed. For both time windows, the dip profile appears to show a shallower ingress slope compared to the egress slope. For the stronger, better defined dips, we measure a mean width (FWZI) of 0.195 days, or as a fraction of the period, (dip duration)/period $=0.106$.

\subsection{EPIC 205024957}

EPIC 205024957 is the one star in our sample that appears to have a significant $24 \mu \mathrm{m}$ IR excess; however, as originally concluded by Carpenter et al. (2009), the lack of an excess at shorter IR wavelengths indicates that this is likely a debris 

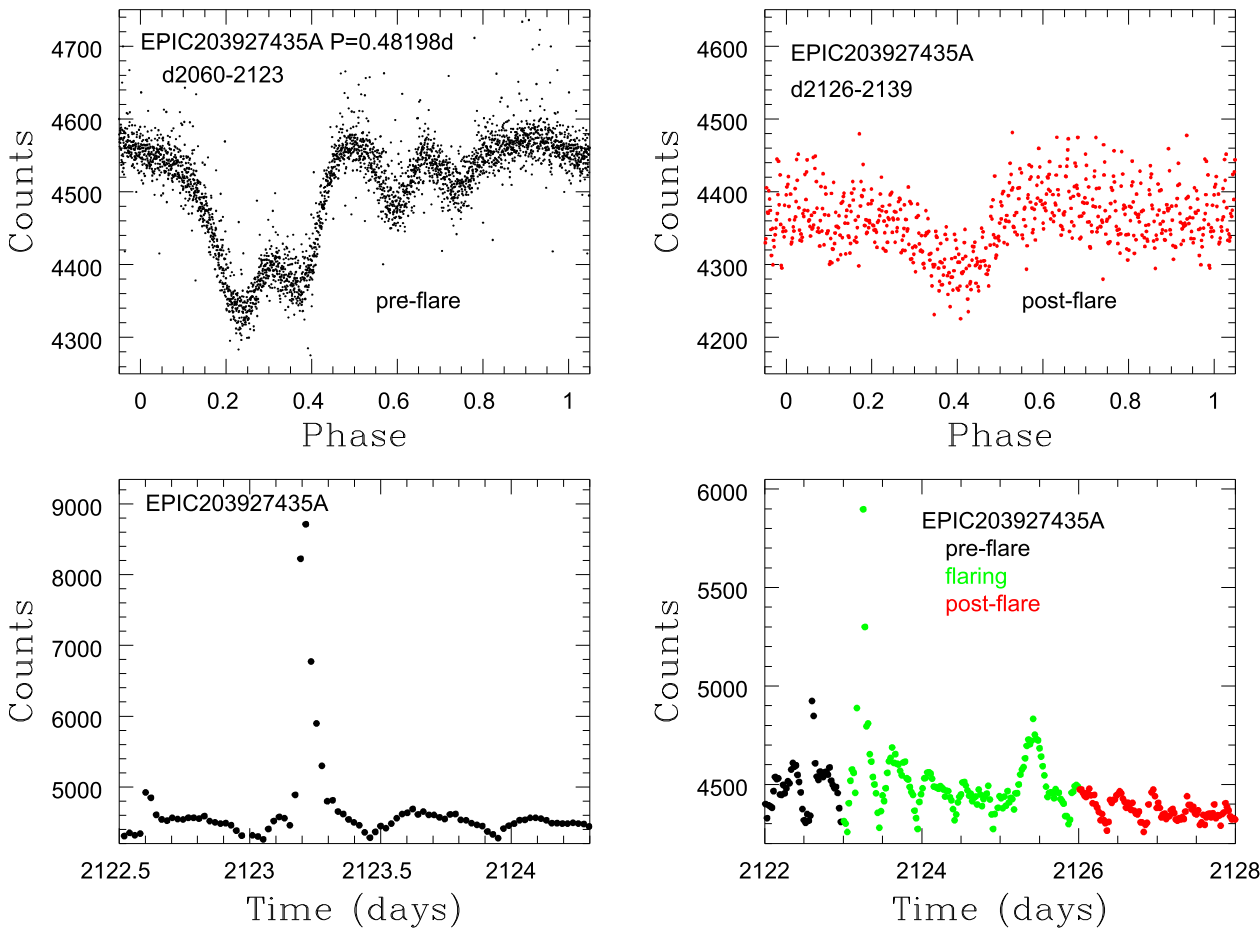

Figure 11. Phased light curve of EPIC 203927435A, illustrating the abrupt change in shape at day 2123, coincident in time with a strong flare.

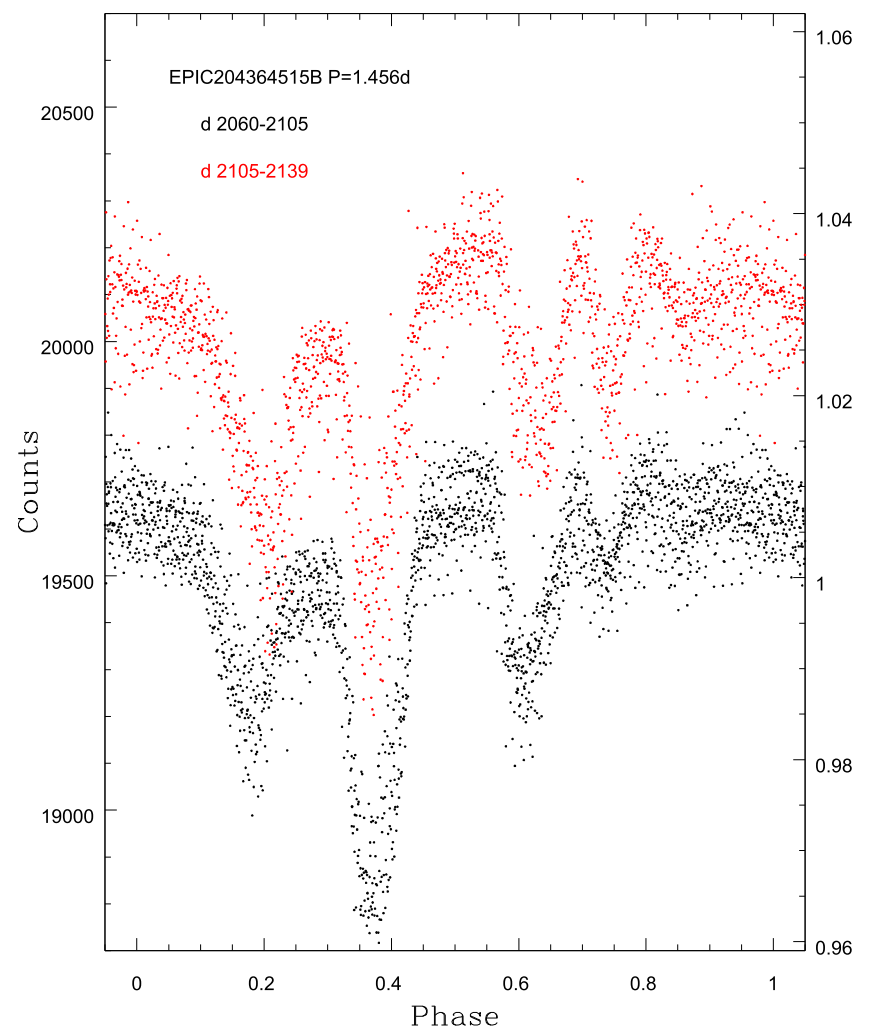

Figure 12. Phased light curve of EPIC 204364515B. There is little change in the shape or depth of the central two dips over the $K 2$ campaign. The left-most dip appears to move to longer phase as time progressed and the right-most dip became stronger in the latter half of the campaign. The rhs $y$-axis normalized count labels are derived for the first time interval (black points).

disk and not a primordial disk. Its weak $\mathrm{H} \alpha$ emission (Table 1) also supports it being a WTTS and not a CTTS. The Lomb-Scargle periodogram for EPIC 205024957 shows a single strong peak at $P=1.6672$ days, largely driven by a nearly sinusoidal spotted-star waveform; however, the phased light curve also shows a definite but fuzzy flux dip (Figure 17). We have analyzed the light curve for this star in the same way as for EPIC 205046529 and reached similar conclusions. That is, we believe the fuzzy appearance of the flux dip is due to both short- and long-term variations in the dip depth, with little contribution from variations in the occurrence times for the dips. Figure 18 illustrates the shortand long-term variability of the flux-dip depths with four 10day portions of its light curve. Based on Gaussian fits to the well-detected flux dips, we derive a best period for the dips of $P=1.6656$ days. For the stronger, better defined dips, we measure a mean dip width (FWZI) of 0.203 days, or as a fraction of the period, (dip duration) $/$ period $=0.122$. Times and depths for the individual flux dips we could measure are provided in Table 4. As for EPIC 205046529, in Figures 19 and 20 we also provide histograms of the dip depths and phased light curves for EPIC 205024957 in two time windows to illustrate the evolution of the dip character during the $K 2$ campaign.

\subsection{EPIC 204143627}

The Lomb-Scargle periodogram for EPIC 204143627 shows a single strong peak at $P=1.125$ days, largely driven by a nearly sinusoidal spotted-star waveform; however, the phased light curve also shows a definite but weak flux dip (Figure 21). We analyzed the light curve for this star in the same way as for EPIC 205046529 and reached similar, though more limited, conclusions due to the lower $\mathrm{S} / \mathrm{N}$ for this star. That is, we believe the fuzzy appearance of the flux dip is due to variations in the dip depth, with little contribution from variations in the occurrence times for the dips. The four panels of Figure 21 show that the flux dip was present and well defined in the first 20 days of the $K 2$ campaign and slowly diminished in depth 
Table 2

Quantitative Measures for Persistent Flux Dips

\begin{tabular}{lcccc}
\hline \hline EPIC ID & Dip Number & Phase & FWZI $^{\mathrm{a}}$ & Dip Depth $^{\mathrm{b}}$ \\
\hline $204296148 \mathrm{~A}$ & 1 & 0.32 & 0.16 & 3.0 \\
$204296148 \mathrm{~A}$ & 2 & 0.62 & 0.11 & 1.4 \\
$204364515 \mathrm{~B}$ & 1 & 0.19 & 0.12 & 1.5 \\
$204364515 \mathrm{~B}$ & 2 & 0.38 & 0.11 & 3.1 \\
$204364515 \mathrm{~B}$ & 3 & 0.62 & 0.11 & 1.9 \\
$204364515 \mathrm{~B}$ & 4 & 0.74 & 0.07 & 0.9 \\
202873945 & 1 & 0.10 & 0.09 & 0.8 \\
202873945 & 2 & 0.42 & 0.10 & 2.3 \\
202873945 & 3 & 0.63 & 0.09 & 1.2 \\
$203962559 \mathrm{~W} 1$ & 1 & 0.37 & 0.08 & 3.2 \\
$203962559 \mathrm{~W} 1$ & 2 & 0.52 & 0.06 & 0.9 \\
$203962559 \mathrm{~W} 1$ & 3 & 0.67 & 0.11 & 3.1 \\
$203962559 \mathrm{~W} 1$ & 4 & 0.87 & 0.15 & 3.5 \\
$203962559 \mathrm{~W} 2$ & 1 & 0.65 & 0.12 & 3.2 \\
$203962559 \mathrm{~W} 2$ & 2 & 0.85 & 0.15 & 2.2 \\
203692610 & 1 & 0.19 & 0.06 & 2.5 \\
203692610 & 2 & 0.29 & 0.06 & 2.5 \\
203692610 & 3 & 0.41 & 0.13 & 7.5 \\
203849738 & 1 & 0.13 & 0.19 & 3.9 \\
203849738 & 2 & 0.79 & 0.07 & 1.2 \\
$205374937 \mathrm{~A}$ & 1 & 0.17 & 0.16 & 3.2 \\
$205374937 \mathrm{~A}$ & 2 & 0.60 & 0.12 & 3.0 \\
$203927435 \mathrm{~A}$ & 0.26 & 0.2 & 4.5 \\
$203927435 \mathrm{~A}$ & 0.39 & 0.2 & 3.6 \\
$203927435 \mathrm{~A}$ & 0.63 & 0.14 & 1.4 \\
$203927435 \mathrm{~A}$ & 0.78 & 0.14 & 1.3 \\
\hline & & & &
\end{tabular}

Notes.

${ }^{\text {a }}$ Full-width, zero-intensity values.

b Note that for the stars that we interpret as likely physical binaries because of the presence of two significant peaks in the Lomb-Scargle periodogram (EPIC 204296148, 204364515, and 203927435), we do not know the relative contribution to the total counts for the two stars. We can therefore derive the amplitude in counts for the flux dip, but when stated as a percent in depth this is just a lower limit. If the two stars are similar in brightness, then the reported depths would be half of the true value. For EPIC 203692610, the measured dip properties are for the time window when the dips were strongest (day 2065 to 2089).

over the duration of the campaign. For the first time window in Figure 21, we measure a dip width (FWZI) of 0.06 days, or relative to the period, (dip duration $) /$ period $=0.055$.

\subsection{EPIC 205483258 (RIK-210)}

EPIC 205483258 is a relatively anonymous (only two references in SIMBAD as of this date) Upper Sco member. Its literature name is RIK-210 (Rizzuto et al. 2015). It is both the earliest type star in Table 1 and has the longest period. Its phased light curve is dominated by a well-defined stable sinusoidal variation presumably due to a highly non-axisymmetric spot distribution. Superposed on that waveform is a single strong narrow flux dip-as shown in Figure 22. The dip width (FWZI) varies from epoch to epoch, but is about 0.4-0.5 days, or relative to the period, (dip duration)/period $\sim 0.08$. The dips are generally asymmetric, with a shallower and more variable ingress profile.

We have much more follow-up data for RIK-210 than for any of our other stars. In David et al. (2017), we provide a detailed examination of the $K 2$ light curve for RIK-210 and an analysis of synoptic spectra for it, which we obtained with

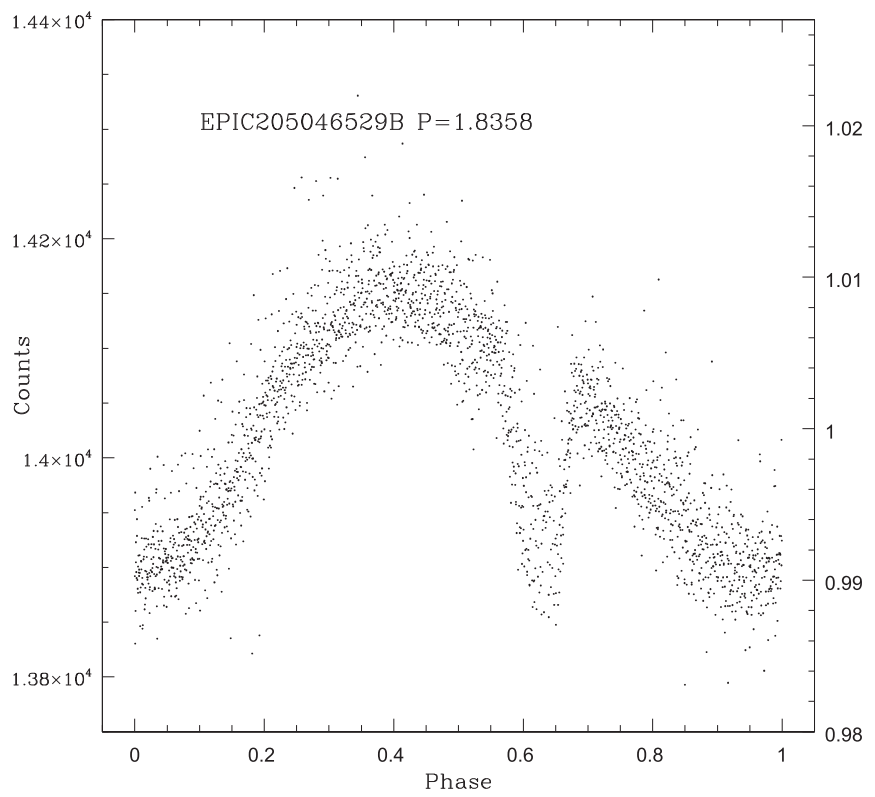

Figure 13. Phased light curve of EPIC 205046529B. The edges of the dip are quite sharp, but the interior of the dip appears filled in. This is primarily because the dip depths vary significantly with time-as illustrated in the next figure.

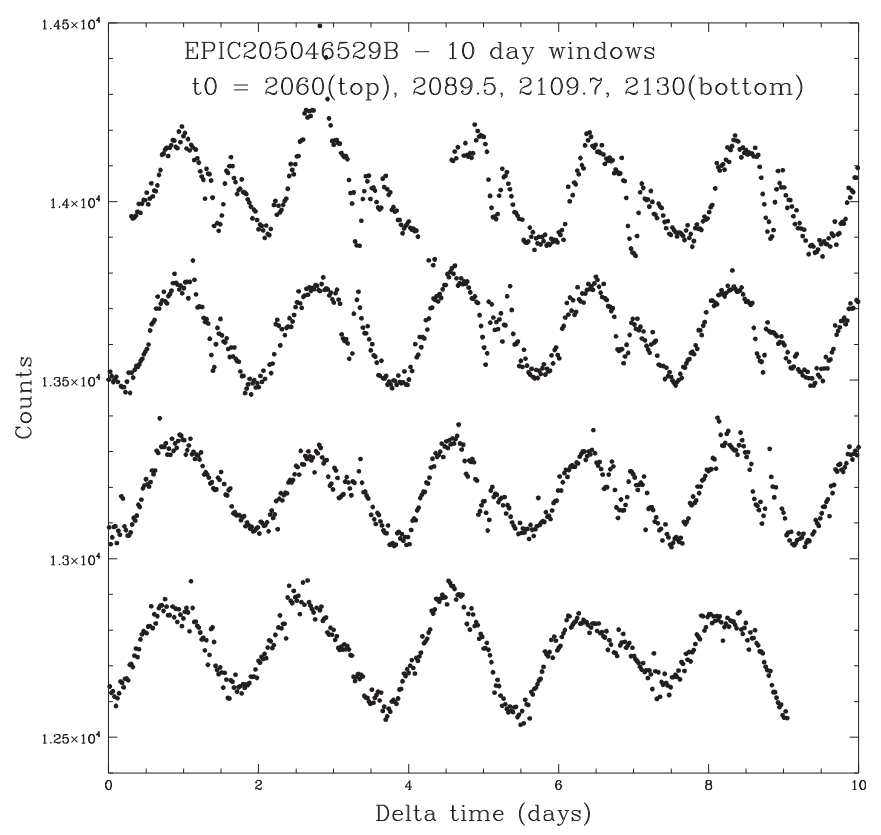

Figure 14. Four 10-day segments of the light curve of EPIC 205046529B. The four segments (top to bottom) are d2060.0-2070.0, d2089.5-2099.5, 2109.7-2119.7, and d2130-2139; they are aligned in phase in $\mathrm{x}$ and displaced arbitrarily in $y$ in order to allow easy comparison of the flux dips. The dips are present but variable in strength in the first three time windows and completely absent from the last time window

Keck HIRES. Radial velocities at 15 epochs exclude any EB scenario to explain the narrow flux dips. The variability in the shape, depth and duration of its flux dip can only be explained by some type of cloud of material orbiting the star at the Keplerian co-rotation radius and with physical dimension similar in size to the stellar radius. We return to the implications of these data in Section 8. 
Table 3

Times and Depths for Flux Dips in EPIC 205046529B

\begin{tabular}{lccc}
\hline \hline Date & Dip Depth (percent) & Date & Dip Depth (percent) \\
\hline 2061.45 & 1.5 & 2087.18 & 1.2 \\
2063.31 & 1.6 & 2089.05 & 0.4 \\
2065.14 & 1.3 & 2090.89 & 1.0 \\
2066.99 & 1.4 & 2092.69 & 0.8 \\
2068.81 & 1.3 & 2094.52 & 1.0 \\
2070.66 & 1.4 & 2096.34 & 0.8 \\
2072.48 & 1.8 & 2098.19 & 0.9 \\
2074.31 & 1.3 & 2100.04 & 0.9 \\
2076.15 & 1.5 & 2101.87 & 1.0 \\
2083.50 & 1.4 & 2103.70 & 1.2 \\
2085.34 & 1.6 & 2105.52 & 1.1 \\
\hline
\end{tabular}

\subsection{Shared Characteristics of the Stars with Transient Flux Dips}

All of the stars in this class share the characteristics below.

1. All four stars have $P>1$ day. In all four cases, there is a well-defined spotted-star waveform in addition to the flux dip, and both share the same period to within the uncertainties. Therefore, for these stars, we are certain that the flux-dip period is the same as the rotation period.

2. All have M spectral types (M2.5 to M5).

3. In all cases, there is only one major flux dip per star. The maximum depths are $1.5 \%, 2.5 \%, 3 \%$, and $20 \%$ (the latter for RIK-210). The widths (full width zero intensity) are on the of order 0.1 in phase or slightly less, corresponding to about $10 \mathrm{hr}$ for RIK-210 and to a few hours for the other three stars.

4. The dip depths vary significantly on both short (cycle to cycle) and long (campaign duration) timescales. From one cycle to the next, the dip can go from present and near maximum depth to absent.

5. In one case for sure, and probably in two of the other cases, the dip shape is asymmetric, with the slope of the ingress being steeper or shallower than the slope of the egress.

6. Of all 23 stars discussed in this paper, only one has a relatively secure IR excess and that is EPIC 205024957.

\section{Further Characterization}

How do the stars we discussed in the previous three sections compare to other low-mass Upper Sco WTTs in terms of their basic physical properties? Figure 23 provides three such comparisons-a color-magnitude diagram, a spectral-type histogram, and a period-color diagram. The color-magnitude diagram shows that despite their unusual light curves, our stars do not appear to have unusual locations in the $V$ versus $V-K_{s} \mathrm{CMD}$, other than their being concentrated to colors corresponding to mid-to-late $\mathrm{M}$ spectral types. There is also no obvious displacement of one of our groups relative to the other two groups in this diagram (as might have been true if they sampled different age or mass ranges). Spectral types are available for about a third of the 1100 WTTS with $K 2$ light curves, and for $>80 \%$ of the stars in Table 1 . The spectral-type histogram reinforces the conclusion from the CMD that our stars are concentrated to mid-to-late $\mathrm{M}$ dwarfs. A very large fraction

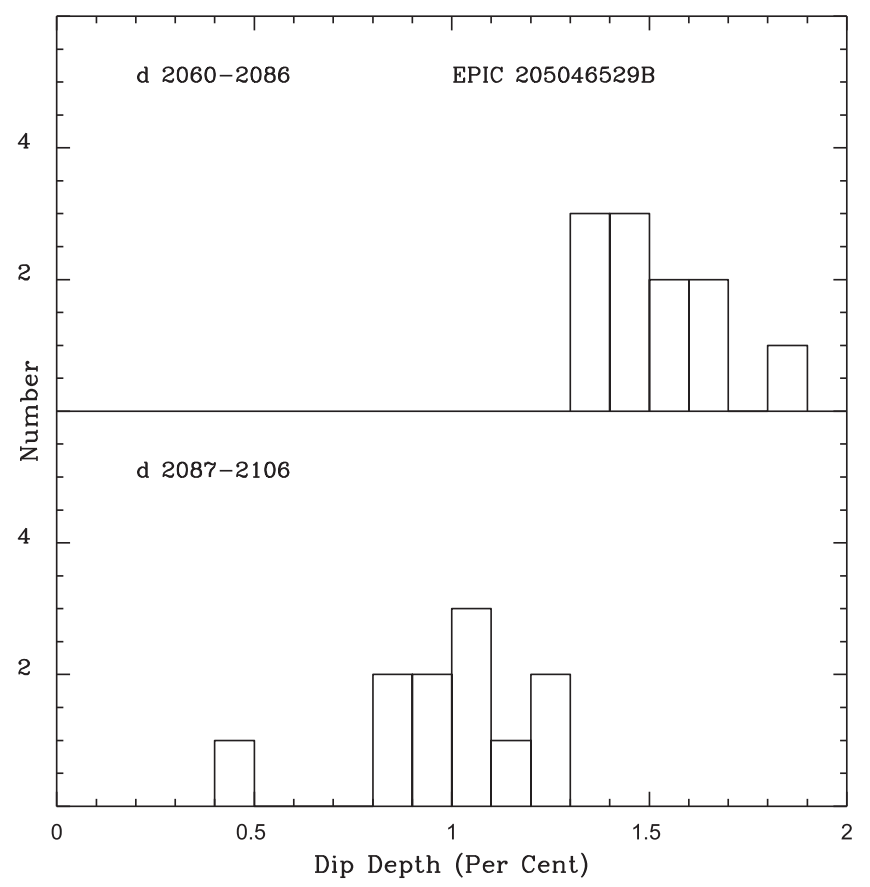

Figure 15. Histograms of the dip depths for EPIC 205046529b for two date ranges-(top) day 2060-2086; (bottom) day 2087-2106. After day 2106, the dip was either not detectably present or was relatively weak.

of the $K 2$ Upper Sco stars are $\mathrm{M}$ dwarfs, so the fact that our stars are $\mathrm{M}$ dwarfs is not unexpected. However, $40 \%$ of the full sample have spectral types earlier than M4, whereas that is true for $<10 \%$ of the stars from Table 1 (i.e., if drawn at random, we would have expected 8 of our 20 stars with spectral type to have to be early M, versus only 2 that we observe).

Conversely, the period-color diagram does show striking correlations. Most importantly, we believe this diagram emphasizes that the stars with scallop-shell light curves segregate to the most rapidly rotating stars in Upper Sco. Several of them have periods quite close to breakup at ages appropriate for Upper Sco. The persistent short-duration fluxdip class are also relatively rapidly rotating on average, but less so than for the scallop shells. The stars with transient shortduration flux dips have periods that are essentially typical of Upper Sco members for their $V-K_{s}$ color.

Figure 24 provides two additional plots, both of which relate to whether the $\mathrm{M}$ dwarfs with unusual phased light curves in Upper Sco have significant signposts of circumstellar disks or active accretion from a disk. The left panel of Figure 24 compares the $[W 1]-[W 3](3.5 \mu \mathrm{m}-12.0 \mu \mathrm{m})$ color of our stars versus the other Upper Sco stars with and without primordial disks. We label our stars as "PEV" in this diagram, for Periodic Exospheric Variables; for the moment, this is simply a conveniently short alias. The PEV stars appear to be quite normal for their $V-K_{s}$ color, and very well separated from the stars of the same mass with primordial disks. The right panel of Figure 24 compares the $\mathrm{H} \alpha$ equivalent widths for the PEV stars to the other stars without obvious IR excesses. With one exception, the PEV stars are again unremarkable. The one exception is EPIC $203185083\left(V-K_{s}=6.6, \mathrm{H} \alpha=-27\right)$. Our conclusion from this is that the types of variability associated with active accretion from a close-in disk (accretion 

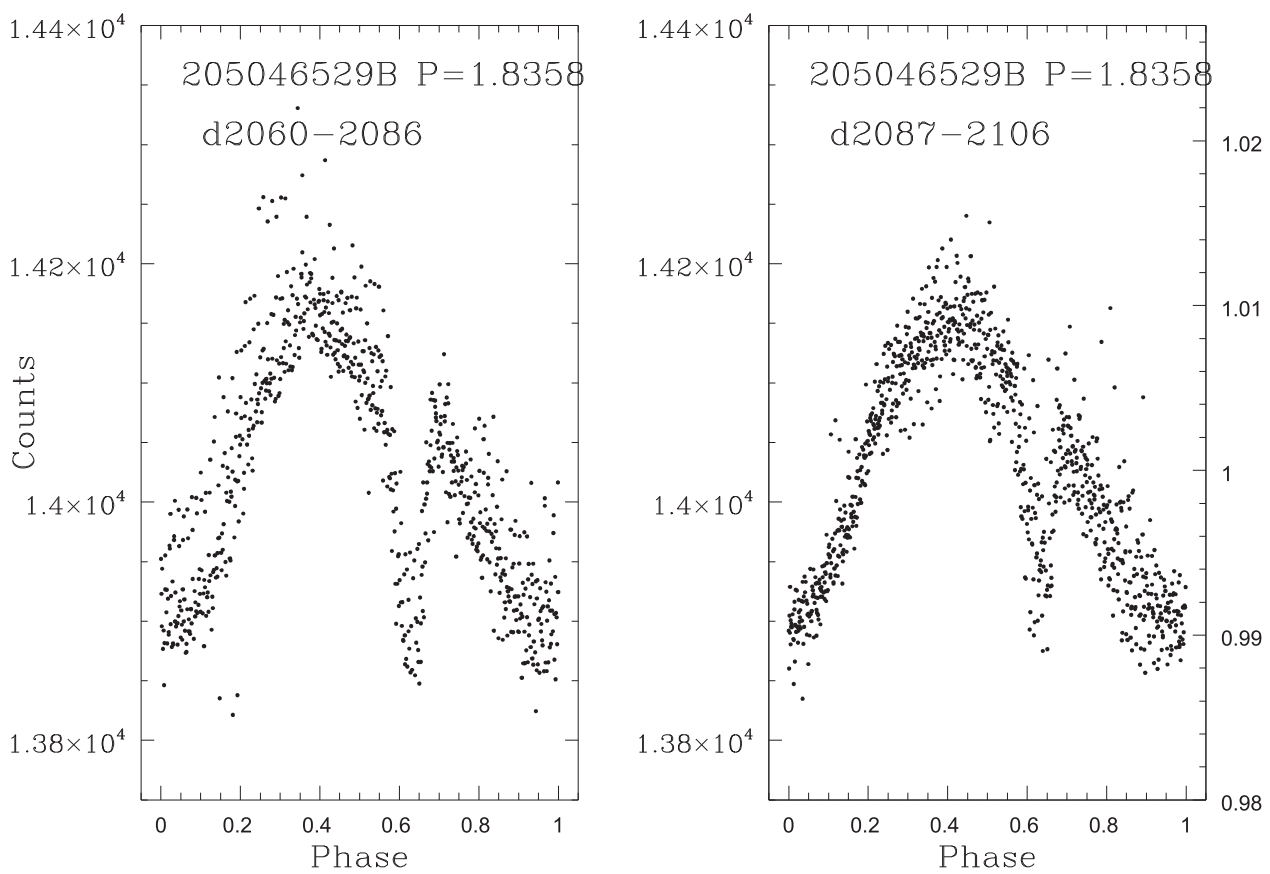

Figure 16. Phased light curve for EPIC 205046529b for the same two time windows as used in Figure 15—(left) day 2060-2086; (right) day 2087-2106.

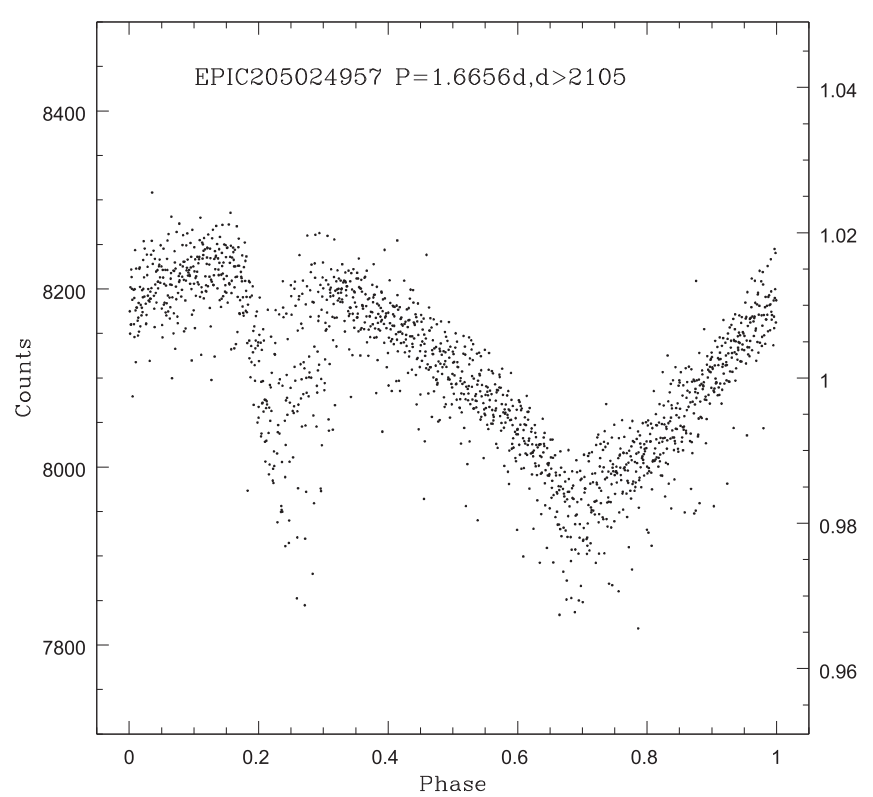

Figure 17. Phased light curve of EPIC 205024957. As for EPIC 205046529B, the edges of the dip are quite sharp but the interior of the dip appears filled in. This is again due primarily to rapid and seemingly random variations in the dip depth with time, as illustrated in the next figure.

hot spots, flux dips due to an inner warped disk, or dust entrained in an accretion stream) are unlikely to be relevant for our stars.

It is also useful to compare the size of our stars to their Keplerian co-rotation radii and to distances from the star where grains would be expected to sublimate. That comparison is provided in Figure 25. For the calculations, we have adopted the Baraffe et al. (2015; hereafter BHAC15) evolutionary models to provide stellar luminosities and radii at $8 \mathrm{Myr}$. The radii in those models may be underestimated because they do

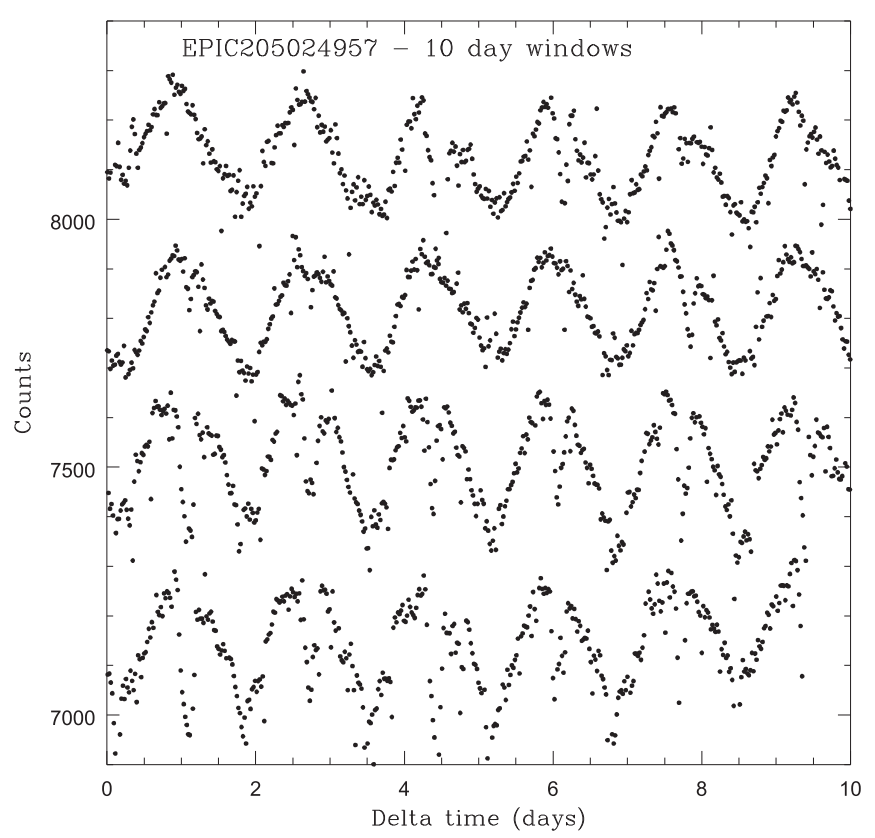

Figure 18. Four 10-day segments of the light curve of EPIC 205024957. From top to bottom, the four time windows shown are d2068.0-2078.0, d2083.0-2093.0, d2119.7-2129.7, and d2129.7-2139; the light curve segments are aligned in phase in $\mathrm{x}$ and shifted arbitrarily in $\mathrm{y}$ in order to highlight the evolution of the flux dips. The dip depths vary greatly on both short and long timescales.

not include "radius inflation" due to magnetic fields or spots (MacDonald \& Mullan 2013; Jeffries et al. 2016; Somers \& Stassun 2017), but we believe the radii are adequate for our current purposes. We use the formula in Monnier \& MillanGabet (2002) to determine sublimation radii, with the two curves corresponding to minimum and maximum $Q_{R}$ values of 1.0 and 4.0, corresponding to a plausible range of sizes for silicate or carbon grains. Based on the spectral types in Table 1, 
Table 4

Times and Depths for Flux Dips in EPIC 205024957

\begin{tabular}{lccc}
\hline \hline DATE & Dip Depth (percent) & DATE & Dip Depth (percent) \\
\hline 2072.48 & 4.3 & 2115.75 & 1.7 \\
2074.13 & 2.5 & 2117.43 & 1.6 \\
2080.78 & 1.0 & 2119.06 & 1.9 \\
2084.11 & 0.9 & 2120.78 & 1.8 \\
2090.83 & 1.6 & 2122.45 & 1.9 \\
2095.9 & 1.0 & 2124.08 & 2.3 \\
2099.08 & 0.8 & 2125.77 & 2.1 \\
2100.75 & 0.7 & 2127.44 & 2.8 \\
2102.43 & 1.0 & 2129.08 & 3.2 \\
2104.1 & 0.7 & 2130.78 & 3.2 \\
2105.75 & 1.6 & 2132.43 & 2.5 \\
2109.1 & 1.5 & 2134.12 & 2.6 \\
2110.8 & 0.9 & 2135.73 & 2.6 \\
2112.5 & 1.0 & 2137.42 & 2.9 \\
2114.1 & 1.3 & $\cdots$ & $\cdots$ \\
\hline
\end{tabular}

most of our stars should have masses about $0.3 M_{\odot}$. The BHAC15 radius for such a star is $0.8 R_{\odot}$ at $8 \mathrm{Myr}$; the corresponding Keplerian co-rotation radii for 0.5 , 1.5 , and 5.5 days are $1.8,3.7$, and $8.7 R_{\odot}$. The curves in Figure 25 show that for the stars with period near 0.5 days, dust is unlikely to be able to form or survive; dust may be able to survive at the Keplerian co-rotation radius for the stars with $P \sim 1.5$ days; and dust would certainly be able to form and survive for extended periods for RIK-210, the star with $P \sim 5.5$ days.

For the persistent and transient short-duration flux-dip stars, the duration, shape, and depth of the flux dip encodes information on the size and nature of the object that is transiting (or being eclipsed by) the star, if that is the correct physical explanation for their variability. For a point source at the Keplerian co-rotation radius $\left(R_{\mathrm{co}}\right)$ whose orbit passes through the Earth-star line, the duration of a transit (or eclipse) is just

$$
\text { duration }=\frac{2 R_{*}}{\left(2 \pi R_{\mathrm{co}}\right)} P_{\text {rot }} .
$$

For radii and masses in solar units and rotation periods in days, the durations are just

$$
\text { duration }=0.076 R_{*}\left(P_{\text {rot }} / M_{*}\right)^{0.333} .
$$

For an adopted age of $8 \mathrm{Myr}$, our stars have masses from the BHAC15 models ranging from about 0.2 to $0.6 M_{\odot}$, and corresponding radii of 0.64 to $1.02 R_{\odot}$.

Figure 26 plots the dip durations we have measured in Sections 5 and 6 for the short-duration flux-dip stars as a function of their periods and compares those data to the duration predicted for a point source for stars with mass of 0.2 and $0.6 M_{\odot}$. For the shortest periods, most of the stars have their maximum observed dip durations consistent with the prediction for a point source; for periods longer than about 1.5 days, however, the observed durations are significantly longer than the point-source prediction, indicating that the occulting (occulted) object has a size comparable to the star. The relationship between dip duration and period appears tight based on the limited amount of data that exists, but is clearly much steeper than our simple Keplerian model.

To illustrate this point in another way, we have estimated stellar radii for each of the persistent and transient flux-dip

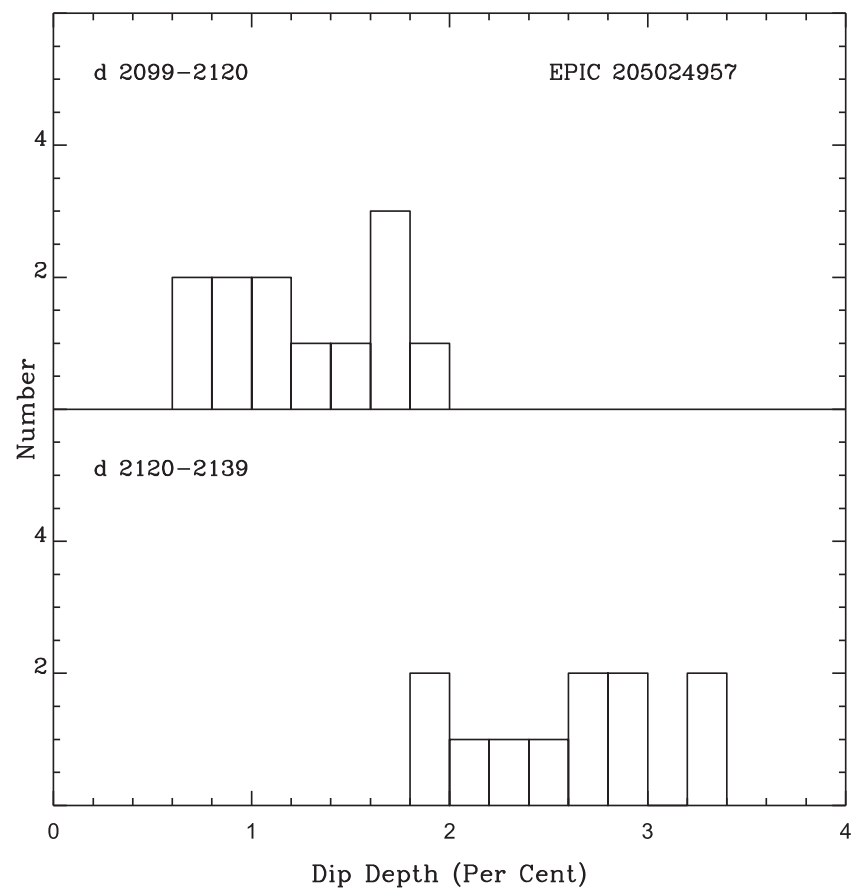

Figure 19. Histograms of the dip depths for EPIC 205024957 for two date ranges-top: day 2099-2120; bottom: day 2120-2139. Prior to day 2099, the dip was either not detectably present or was relatively weak.

stars. For that, we have used our spectral types and estimated $V-K_{s}$ color and Table 6 of Pecaut \& Mamajek (2013) to provide intrinsic $T_{\mathrm{eff}}, B C_{J}$, and $V-K_{s}$ color. Those data and the Stefan-Boltzmann law yield direct stellar radius estimates; for stars identified as binaries in Table 1, we divided the system luminosity by two prior to calculating the radius. We then derive estimates of the Keplerian co-rotation radius, adopting masses from our estimated luminosities and the BHAC15 $8 \mathrm{Myr}$ isochrone. The maximum duration for a point source at the Keplerian co-rotation radius then follows from the formula above. Table 5 provides our estimated stellar radii and compares the predicted maximum dip durations to the longest duration flux dips for each star. The uncertainties in the stellar radius estimates, and hence in the durations, from propagating uncertainties in the distance and age and in the stellar properties are likely $15 \%-20 \%$ (see, for example, similar calculations for individual planet-hosting stars by Mann et al. 2016 and David et al. 2017). Because radii two to three times as large as we estimate would be required for the dip durations to be consistent with observations for several of our stars (including three of four of the transient dip class), we conclude that radius inflation due to magnetic effects (e.g., MacDonald \& Mullan 2017) cannot explain these discrepancies and instead that the transiting/occulting body must have size comparable to the star.

The shape of the flux dips can also provide qualitative information on the nature of the occulting body. The roughly triangular flux dips for our transient flux-dip group are roughly similar in shape to the flux-dip shapes found in many eclipsing binaries (EBs). In most EBs, the flux dips are very stable in their depth and shape and the dip profile is symmetric. However, if the primary of the EB were heavily spotted, the flux dips could be asymmetric if the transit path crosses regions of higher or lower spot coverage. If, in addition, the EB orbit is 

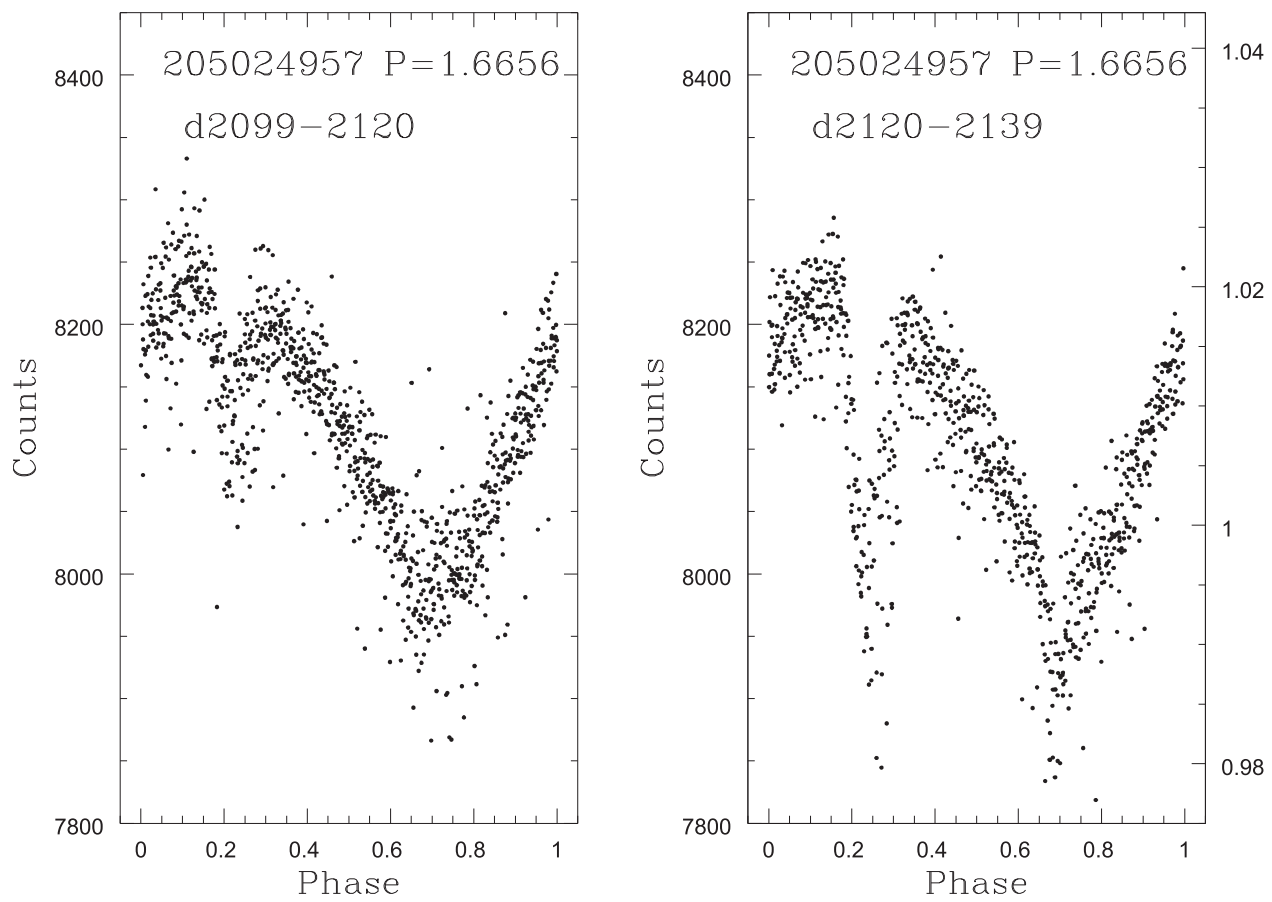

Figure 20. Phased light curve for EPIC 205024957 for the same two time windows as used in Figure 19—(left) day 2099-2120; (right) day 2120-2139.

precessing and the spots are changing on timescales comparable to the orbital period, flux dips with variable depth and shape could result. Our four transient flux-dip stars are indeed heavily spotted. However, the spotted-star waveforms are very stable over the entire $K 2$ campaign. Therefore, even a precessing EB orbit would at most produce changes in the dip shape and depth that vary smoothly with time, which is not the case for our stars.

The combination of dip depths of a few percent, and the above arguments from durations and shapes therefore suggests that the occulting (occulted) bodies are likely to have sizes comparable to that of the star and optical depths (or surface brightnesses or filling factors) that are small.

One can also draw inferences as to the physical mechanism (s) driving the variability in our group of stars from the presence or absence of similar light-curve morphologies in surveys of the light curves of other samples of $\mathrm{M}$ dwarfs. First, to our knowledge, none of the $\sim 3000 \mathrm{M}$ dwarfs in the original Kepler field show light-curve morphologies like those in our three groups (McQuillan et al. 2013). This likely arises both from the relative paucity of mid-to-late M dwarfs in the Kepler sample, and to a paucity of young and/or rapidly rotating $\mathrm{M}$ dwarfs. Second, $K 2$ light curves have been obtained for several hundred mid-to-late $\mathrm{M}$ dwarfs in both the Pleiades (age $\sim 125 \mathrm{Myr}$ ) and Praesepe (age $700 \mathrm{Myr}$ ) open clusters. We (Rebull et al. 2016) found six late M dwarfs in the Pleiades with persistent, short-duration flux dips; we find no such stars in the older Praesepe cluster. We have found no examples of scallop-shell waveforms nor transient short-duration flux dips in either the Pleiades or Praesepe. ${ }^{15}$ We discuss the implications of these results in the next section.

\footnotetext{
${ }^{15}$ In the same mass range as for Upper Sco, there are about 250 Pleiades stars with $K 2$ light curves and period $<0.65$ days, and about 400 Pleiades stars with period $<2$ days. For Praesepe, in that same mass range, there are about 50 stars with period $<0.65$ days and about 200 stars with period $<2$ days.
}

\section{Physical Interpretation}

Light curves from CoRoT, Kepler, and K2 have identified a small but growing population of stars with photometric variability that often seems mysterious and often takes the form of short-duration flux dips. This small menagerie of objects includes pre-main-sequence stars (e.g., Cody et al. 2014), but also includes main-sequence stars (e.g., Rappaport et al. 2012; Boyajian et al. 2016) and even white dwarfs (Vanderburg et al. 2015). Possible physical mechanisms for producing the short-duration flux dips range from occultations by warped or structured circumstellar disks to dust streaming away from a disintegrating close-in planet (as well as other even more speculative thoughts). In many cases, a consensus has not yet been reached as to which mechanism is responsible for the observed variability.

Our goal for this paper has been to add a new set of objects to this menagerie and to provide detailed empirical data for these stars in order to begin the process of categorizing their variability and placing them in the context of the existing objects with similar properties. In this section, we briefly consider several broad categories of physical models as possible mechanism to explain our light curves. We expect that this is just the beginning of a lengthy process, to which we hope many other groups will contribute.

\subsection{Could Cool Spots Be Responsible for the Variability Signatures We Have Identified?}

Non-axisymmetrically distributed spots on the surfaces of low-mass stars typically produce relatively simple and smooth phased light-curve shapes with one or two maxima and one or two minima per period. However, it is not inconceivable that some combination of spot sizes and locations might produce light-curve signatures similar to our unusual Upper Sco stars. In order to address this hypothesis, we created a spotted-star modeling routine and produced simulated light curves for a 

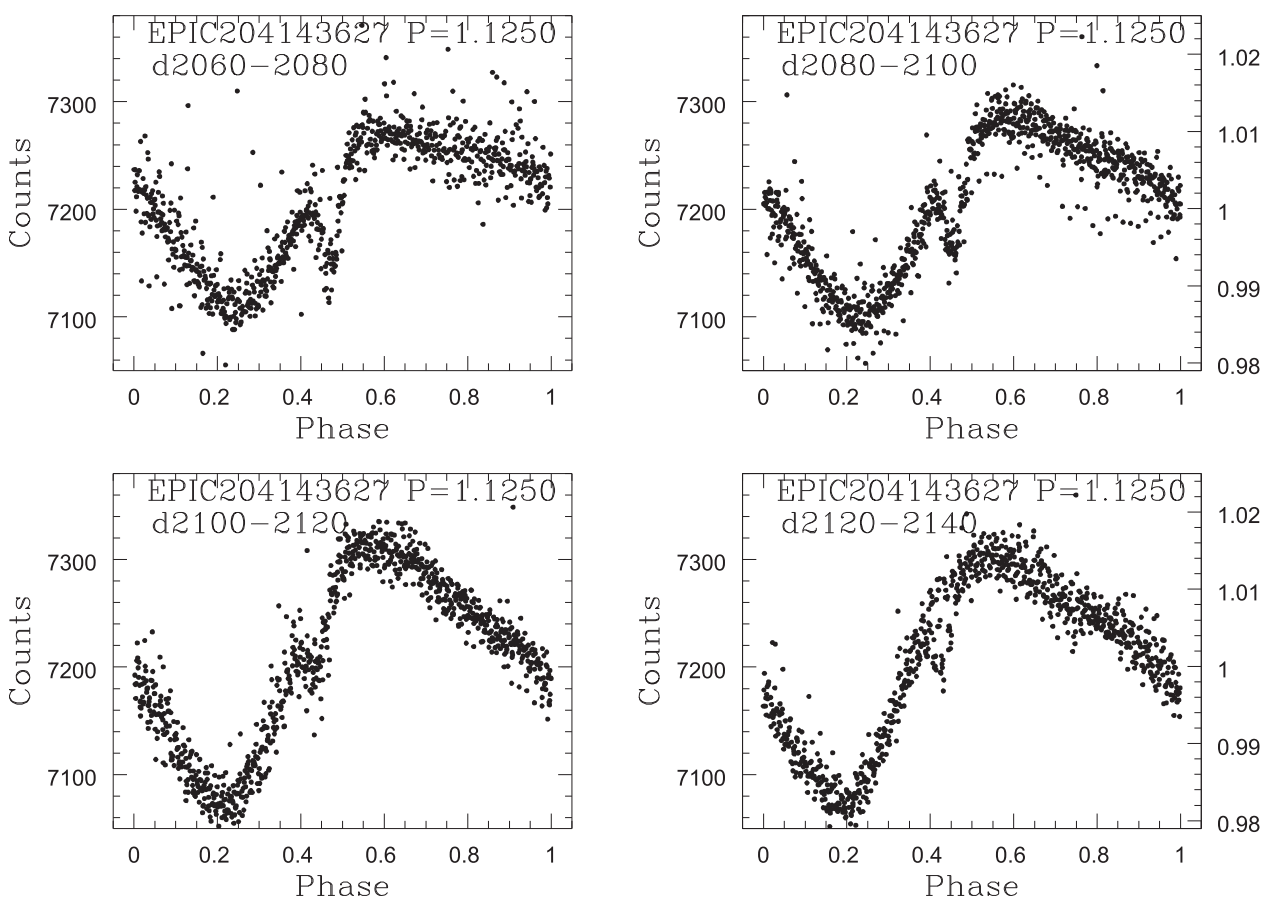

Figure 21. Phased light curve of EPIC 204143627. The four panels show successive 20-day sections of the day, all phased to a period of 1.125 days. The flux dip at phase about 0.45 became less distinct and shallower as the campaign progressed.

variety of spot configurations. We describe the spot simulation program and some of the tests we have run in the Appendix. Based on those simulations, it is possible to get light-curve shapes that are very similar to the scallop-shell and persistentdip classes if one puts spots very close to the upper (or lower) limb of the star as viewed from Earth. However, the spot distributions that can mimic the light-curve shapes cannot also yield light-curve amplitudes that match our observations. For several of the scallop shells, the full amplitude is on the order of $10 \%$, and for a number of the persistent flux-dip class, the dip depths are between $3 \%$ and $8 \%$ with FWZI $<0.2$ in phase. In order to yield the very structured phased light curves of the scallop-shell class, or the very narrow (in phase) flux dips of the persistent-dip class, the spots have to be very near the edge of the visible hemisphere of the star. Because of the geometric fore-shortening and limb darkening when at the edge of the visible hemisphere, such spots yield amplitudes or dip depths less than $1 \%$, even if the spots are assumed to be totally black. Larger spots or spots further from the "terminator" yield light curves that are smoother and do not match the morphologies we see. Therefore, we believe that cool spots on the stellar surface do not provide a suitable model to explain the lightcurve morphologies for our Upper Sco stars.

\subsection{Could Variable Extinction from a Primordial Disk Explain Some of Our Light Curves?}

There is some similarity between the light curves of some of the stars in Table 1 and some of the stars identified in recent years as "dippers" in Orion, NGC 2264, and Upper Sco (Morales-Calderon et al. 2011; Cody et al. 2014; Stauffer et al. 2015; Ansdell et al. 2016); these stars are also sometimes referred to as AA Tau analogs (Bouvier et al. 1999; Alencar et al. 2010; McGinnis et al. 2015). The light curves of AA Tau stars show a wide diversity, with most of them showing very broad and structured flux dips that are quite dissimilar from our Upper Sco stars and thus do not serve as plausible comparison objects. A small subset of them, however, do have periodic short-duration flux dips sometimes superposed on sinusoidal variations with the same period (Stauffer et al. 2015), thus superficially linking their light-curve morphology with that of the stars we have discussed in this paper. For these stars, the periodic short-duration flux dips have generally been attributed to extinction by localized structures in or above the star's inner circumstellar disk, where the inner disk rim and stellar photosphere are assumed to be locked into at least approximate co-rotation. Could some/many of the stars in Table 1 simply be another subset of this class, with the same basic physical processes producing the observing photometric variability?

We believe the answer to that question is primarily no, based on one major difference between our stars and the stars of the dipper class. All but two of the 40 dippers in Orion identified by Morales-Calderon et al. and all of the dippers identified in NGC 2264 by Cody et al. and by Stauffer et al. have strong IR excesses and SEDs appropriate for Class I or II YSOs. All of the dippers in Upper Sco identified by Ansdell et al. (2016) have strong $24 \mu \mathrm{m}$ excesses, and all but one of them have strong $12 \mu \mathrm{m}$ excesses. Those IR excesses arise from warm dust at or near the inner edge of their primordial circumstellar disks. As discussed in Section 2.2 and in the Appendix, with one exception, our stars do not have IR excesses and have SEDs consistent with bare photospheres. Also, for most of our stars (with $P<1$ day), the Keplerian co-rotation radius lies interior to the likely dust sublimation radius and therefore any "cloud" responsible for the periodic light-curve variability should be dust free.

The CoRoT light curves for NGC 2264 are similar in quality to the $K 2$ light curves for Upper Sco, and CoRoT light curves were obtained for more than 300 WTT cluster members (about twice as many WTTs as CTTs); however, none of those WTTs 

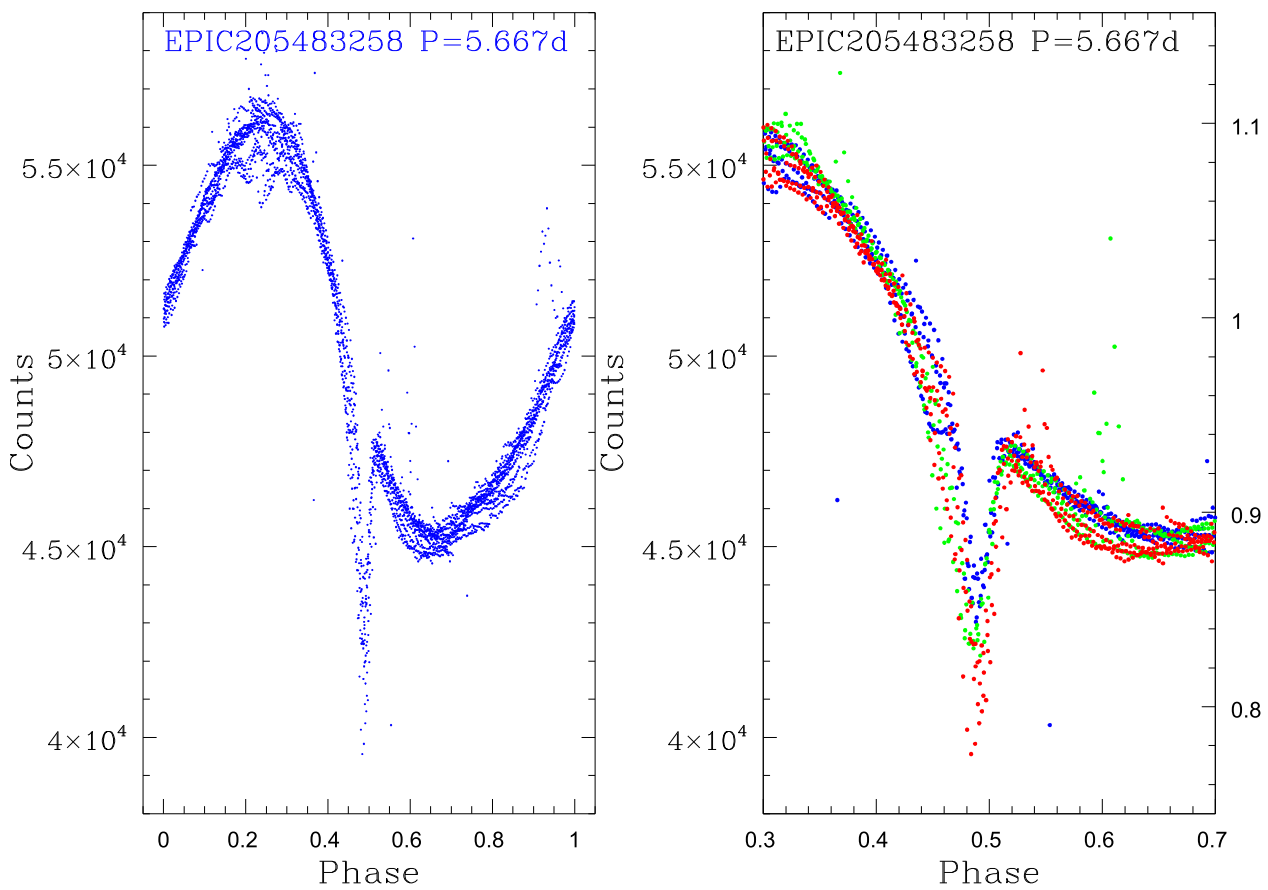

Figure 22. (Left) Phased light curve of EPIC 205483258 (aka RIK-210). (Right) Expanded view of the light curve, highlighting the narrow flux dip. Different colors correspond to different time windows-illustrating that the shape of the flux dip varies significantly on short timescales.

showed light-curve morphologies like our Upper Sco stars in Table 1. Could that absence somehow affect the conclusion we have just reached? Probably not. NGC 2264 is five times further away than Upper Sco, and the CoRoT limiting magnitude is brighter than for $K 2$. Therefore, the sample of stars with good light curves in NGC 2264 contains very few stars in the mass range of the stars in our Table 1 (Venuti et al. 2017 include only two stars with $P<1$ day and mass $<0.4 M_{\odot}$ in their table of NGC 2264 rotation periods from CoRoT). To the extent that the light-curve signatures we see require young ages, rapid rotation, and low masses, we would not expect to have found them in NGC 2264 because we were not able to sample the needed mass range.

\subsection{Could Dusty Debris from Young Forming or} Disintegrating Planets Explain the Variability for Some of Our Stars?

As discussed in Sections 7, 8.2, and in David et al. (2017), the transient short-duration flux dips found for four of our stars cannot be produced by cool spots on the stellar photosphere or by any other plausible phenomenon associated with the surface of the star. The equality of the period for the narrow flux dip and the sinusoidal waveform (which we assume is due to spots) therefore places the material responsible for the narrow flux dips at the Keplerian co-rotation radius. That material must be distributed over a region with size comparable to the star (Figure 26 and Table 5), but have low average optical depth given flux-dip depths generally just a few percent. Several recently discovered stars have light-curve properties that seem reasonably analogous to our four transient flux-dip stars. The closest analog is PTF 08-8695 (van Eyken et al. 2012; JohnsKrull et al. 2016), an M3 star in the $\sim 3$ Myr old 25 Ori subgroup of Orion. Light curves for PTF 08-8695 show narrow flux dips with depths up to $3 \%$, FWZI $\sim 0.18$ in phase, and a period of $0.448 \mathrm{~d}$. The flux-dip depths are variable and at some epochs are not present at all. The dips are superposed on a more or less sinusoidal spotted-star waveform with identical period to that for the flux dips. These properties are remarkably similar to our four Upper Sco transient flux-dip sources. Van Eyken et al. (2012) concluded that the best interpretation of the data was to ascribe the flux dips to transits of a recently formed or forming planet undergoing Roche-lobe overflow mass loss.

Two main-sequence field stars with Kepler or $K 2$ data have light-curve properties that make them somewhat analogous to our transient flux-dip stars. KIC 12557548 (Rappaport et al. 2012 ) is a 16th magnitude, field $\mathrm{K}$ dwarf with a rotation period $P \sim 23$ days and hence a likely age of a few Gyr. The Kepler data show it to have asymmetric flux dips with a period of $15.7 \mathrm{hr}$; the flux dips vary irregularly in depth, with a maximum depth of about $1.3 \%$. The FWZI of the dips is about 0.15 in phase. K2-22b (Sanchis-Ojeda et al. 2015) is a field M0 dwarf with a rotation period $P \sim 15$ days and hence a likely age of about a Gyr. Its $K 2$ light curve shows asymmetric flux dips with a period of about $9.1 \mathrm{hr}$; the flux dips are highly variable in depth, with dip depths from zero to $1.3 \%$. The FWZI of the dips is about 0.09 in phase. The flux dips for both stars have been modeled by the above authors as arising from a planet that is in the process of disintegrating. The surface of each planet would have temperatures near $2000 \mathrm{~K}$. In the Rappaport et al. (2012) model, a dusty wind from the surface generates a dust stream/tail that is responsible for the transits, with the curved geometry of the tail explaining the asymmetric dip profiles and their variability.

If the model proposed for KIC $1255 \mathrm{~b}$ and $\mathrm{K} 2-22 \mathrm{~b}$ is correct, those planets are at a kind of fiery Goldilocks distance from their star-close enough for their surfaces to be molten, but not so close that dust grains could not survive in their planetary wind stream long enough to create a tail of sufficient extent to explain the transit duration and asymmetric shape. For our four transient flux-dip stars, using the parameters in Table 5, the 

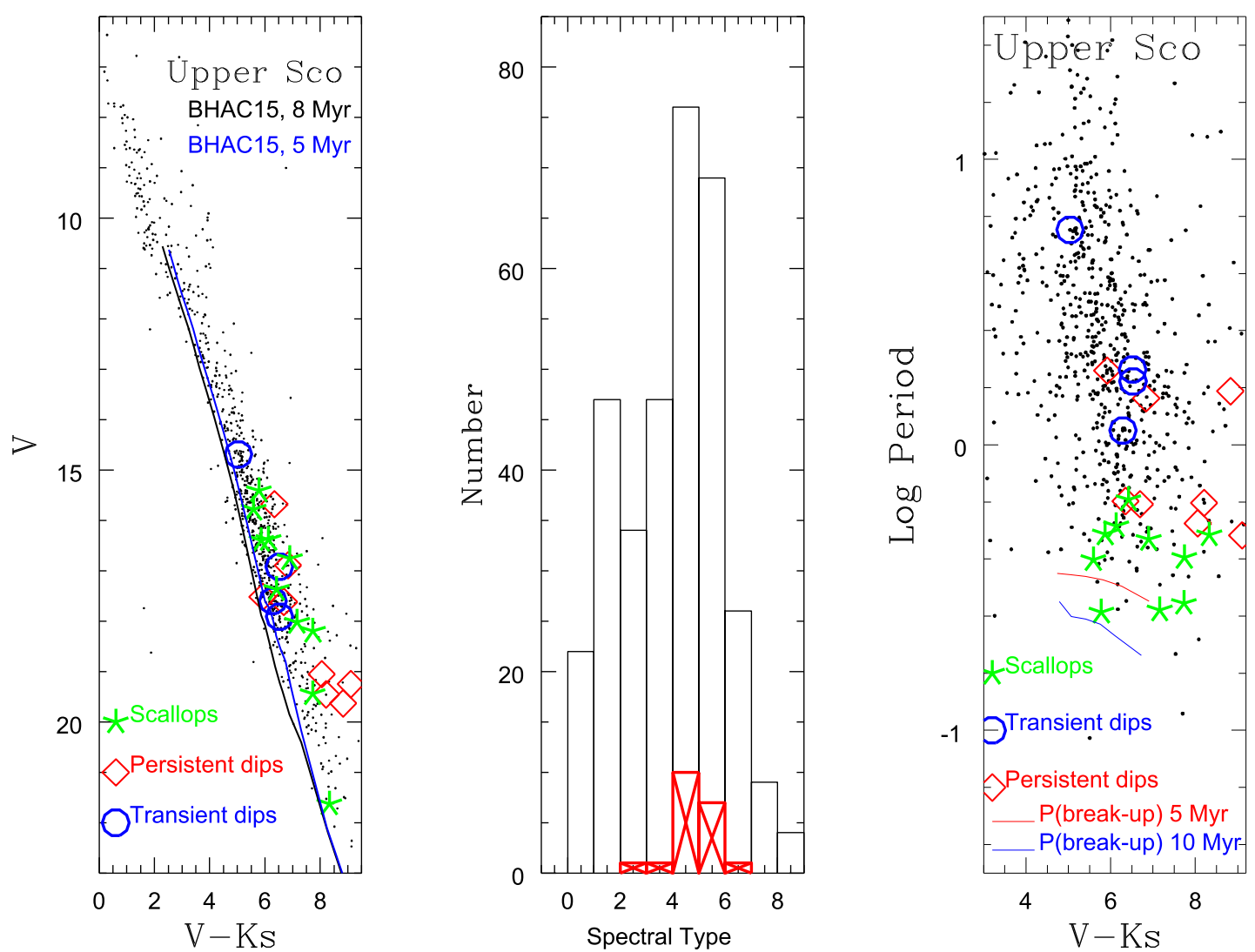

Figure 23. (Left) $V$ vs. $\left(V-K_{s}\right.$ ) color-magnitude diagram for the Upper Sco WTTs with $K 2$ light curves. All of the stars from this paper are in the lower half of the diagram (i.e., mid-to-late $\mathrm{M}$ dwarfs), but we see no striking differences among our three groups. (Middle) Spectral-type histogram for the Upper Sco members with known spectral types (not shown are FGK stars). The $x$-axis are M subtypes (e.g., "0" is M0; " 8 " is M8). The stars with unusual light curves from this paper are shown in red; they preferentially occur among the stars with spectral types M4 and later. (Right) Log Period vs. $V-K_{s}$ diagram for the same set of stars. The most obvious correlation here is that the stars with scallop-shell light-curve morphology have among the shortest periods in Upper Sco; they also have systematically shorter periods than our other two classes. Some of their periods are very near or at the theoretical break-up period for an age of 5-10 Myr.

surface temperature of a hypothetical planet would be in the range $1200-1500 \mathrm{~K}$, and thus the specific mechanism proposed for KIC $1255 b$ and K2-22b is not likely applicable. However, we believe the similarities in light-curve properties between these two stars and our four stars make a compelling case to ascribe the flux dips for our stars to dust associated with a planet. In our case, the distance of the planet from the star is not a function of the star's luminosity but is instead a function of its rotation period and mass. Planets at about that location could arise from orbital migration within a circumstellar disk, and then having that migration stop when the planet reaches the inner rim of the disk - which is expected to be close to the Keplerian co-rotation radius for the star (Papaloizou 2007). What is not obvious is the mechanism for creation of the dusty cloud that would cause the transits for our stars. Several possibilities are explored in David et al. (2017).

\subsection{Could Scattered/emitted Light from Circumstellar Gas Clouds Explain Some of Our Light Morphologies?}

The salient features of the persistent flux-dip class are multiple short-duration flux dips with FWZI 0.1-0.15 in phase and depths of a few percent. Periods are always less than two days, usually less than 1 day. Long-term behavior is akin to the punctuated equilibrium models in evolutionary theory-long periods of stability, but often with abrupt shifts where the depth of one or more dips changes significantly. At some level, the scallop-shell class is just a more extreme version of the persistent flux-dip class-more structure in the phased light curve, shorter periods (all $<0.65$ days), the same or more frequent abrupt shifts in light-curve morphology. We therefore hope to find a single physical mechanism that could explain the light-curve variability of both classes.

The closest analog to these stars we can find in the literature is HHJ 135, in the Pleiades (Rebull et al. 2016). HHJ 135 is a $125 \mathrm{Myr}$, dM4.5 star; its $K 2$ data yields a period of 0.61 days and a phased light curve showing three short-duration flux dips with a very similar appearance to our Upper Sco persistent fluxdip stars EPIC 203692610 and EPIC 204364515. This primarily tells us that extreme youth (low gravity) is not required for this morphological signature.

The next best analogy in the literature we can find for these stars is, surprisingly, an $8 M_{\odot}$ star. $\sigma$ Ori $\mathrm{E}$ is a rapidly rotating Be star. Ground-based and MOST light curves for $\sigma$ Ori E show it to have two short-duration flux dips (widths about 0.2 in phase) superposed on a wave-shaped undulation with a period of 1.19 days (Townsend et al. 2013). The light-curve shape is apparently stable over many years. The MOST light curve closely resembles that for our persistent flux-dip stars EPIC 204296148 and EPIC 205374937. Townsend \& Owocki (2005) proposed that the $\sigma$ Ori $\mathrm{E}$ light curve is a natural outcome for a rapidly rotating star with a wind and a strong magnetic dipole field. Gas from the wind accumulates in a lumpy warped disk 

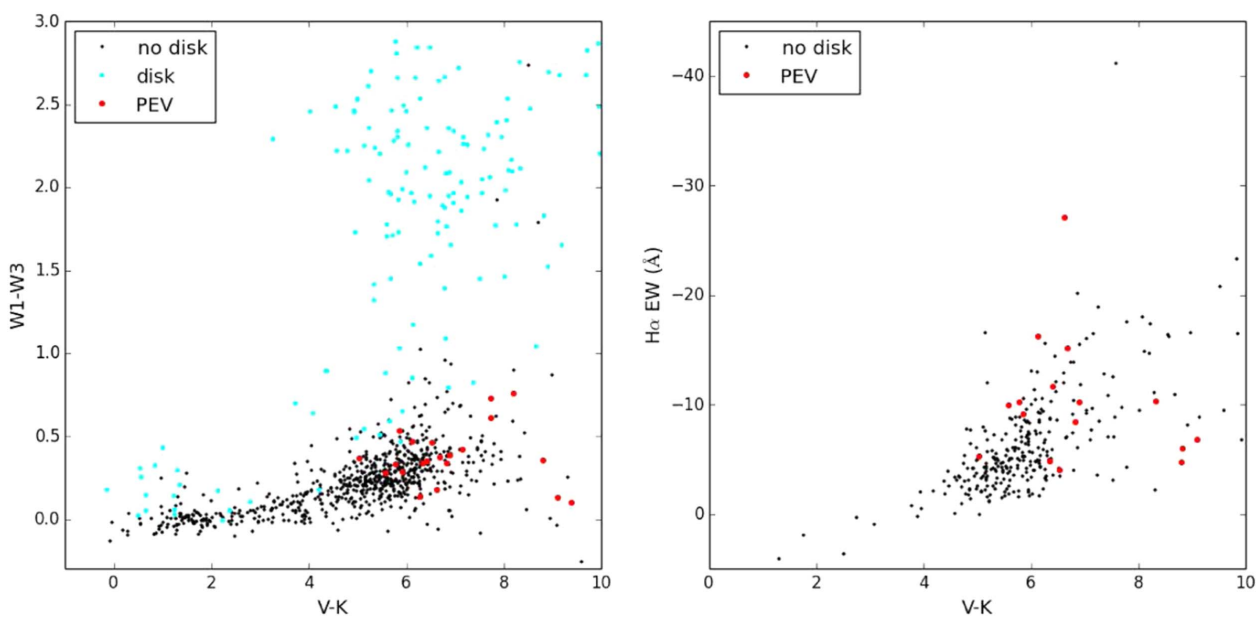

Figure 24. (Left) WISE $W 1-W 3$ color vs. $V-K_{s}$ for Upper Sco members with and without primordial disks (A. M. Cody et al. 2017, in preparation) and the stars from Table 1. The stars without IR excesses are shown as black dots; the stars with strong IR excesses are shown as cyan dots, and the Table 1 stars are shown as red dots. The figure legend refers to the Table 1 stars as PEVs, or Periodic Exospheric Variables. The PEV stars appear to track well the locus of the other WTTs. (Right) Plot of $\mathrm{H} \alpha$ equivalent width vs. $V-K_{s}$ for the stars without IR excesses and the PEV stars. The H $\alpha$ data comes from Preibisch et al. (2001), Slesnick et al. (2008), Lodieu et al. (2011), Rizzuto et al. (2015), and from Table 1. The PEV stars are again normal for their $V-K_{s}$ color, with one exception (EPIC 203185083).

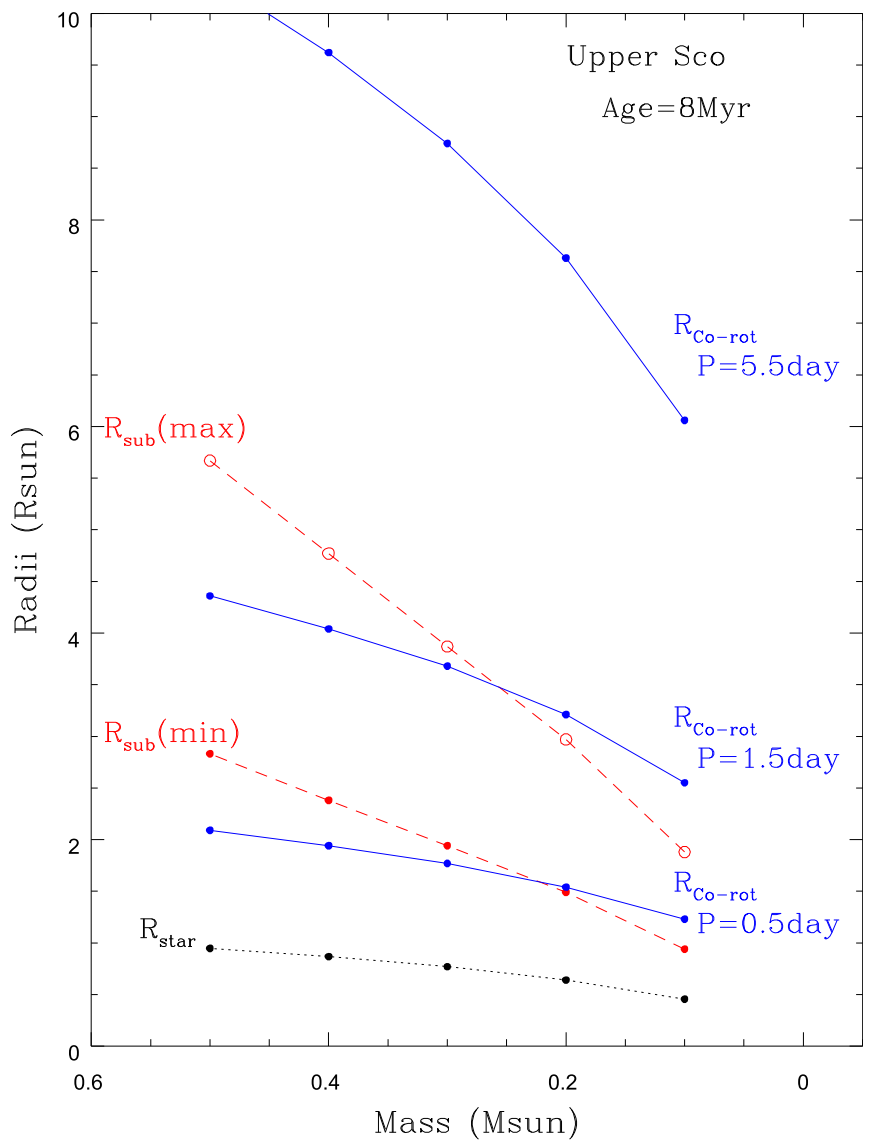

Figure 25. Stellar radius, Keplerian co-rotation radius, and estimated sublimation radii using extremes of grain properties from Monnier \& Millan-Gabet (2002) for an assumed $8 \mathrm{Myr}$ age and using BHAC15 models. The Keplerian co-rotation radius is provided for three rotation periods- $0.5,1.5$, and 5.5 days-which encompasses most of the period range found for the stars discussed in this paper. The black dotted curve provides estimated stellar radii as a function of mass; the blue solid curves are the estimated Keplerian co-rotation radii for three rotation periods that are representative of our Upper Sco stars; and the red dashed curves illustrate approximate sublimation radii as a function of stellar mass.

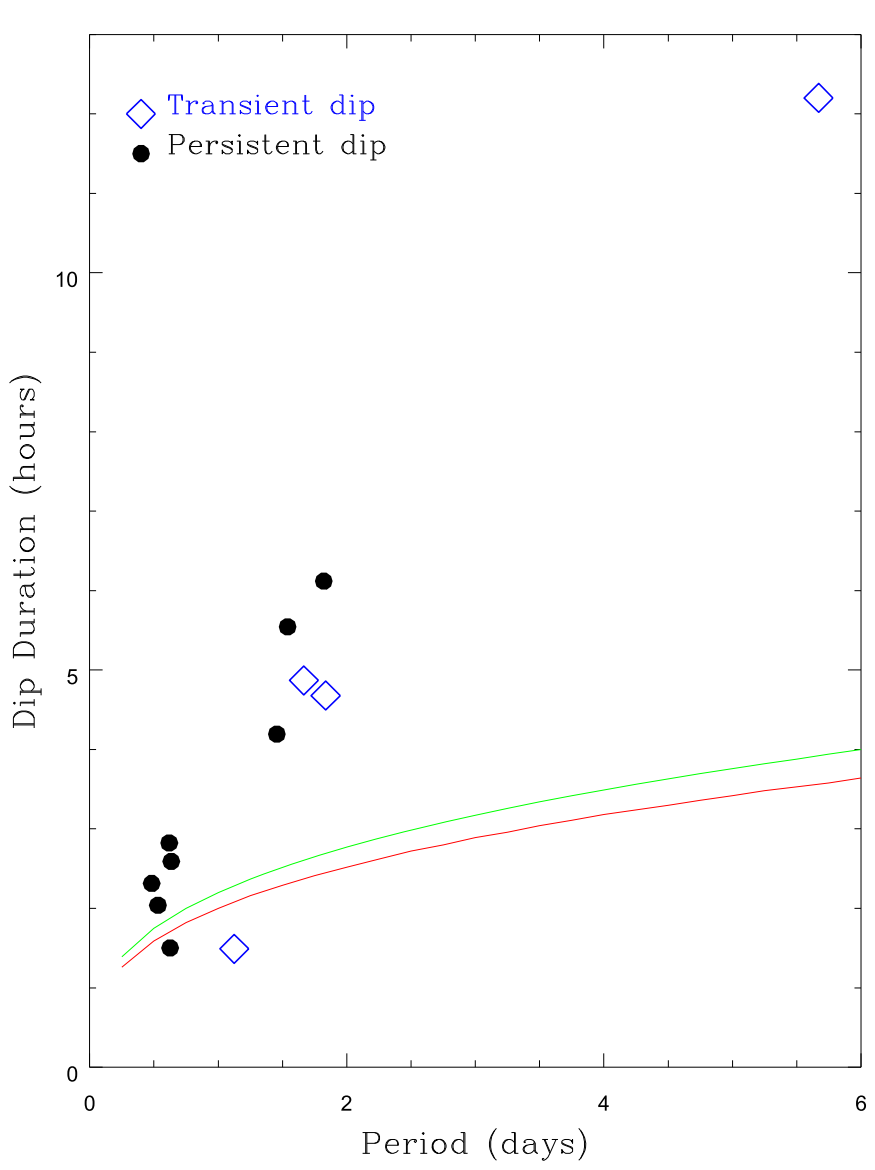

Figure 26. Measured maximum flux-dip duration for our Upper Sco M dwarfs compared to the predicted (maximum) duration for a flux dip due to a pointsource object transiting its parent star (for an age of $8 \mathrm{Myr}$ and mass of 0.2 and $0.6 M_{\odot}$ - encompassing the mass range of our stars). Some of the dips are consistent with the prediction, implying a relatively small size, whereas other dips-particularly for the stars with longer rotation periods-suggest sizes comparable to that of the star. RIK-210 is the outlier to the upper right of the diagram. 
Table 5

Comparison of Dip Durations to Predictions for a Point Source at the Keplerian Co-rotation Radius

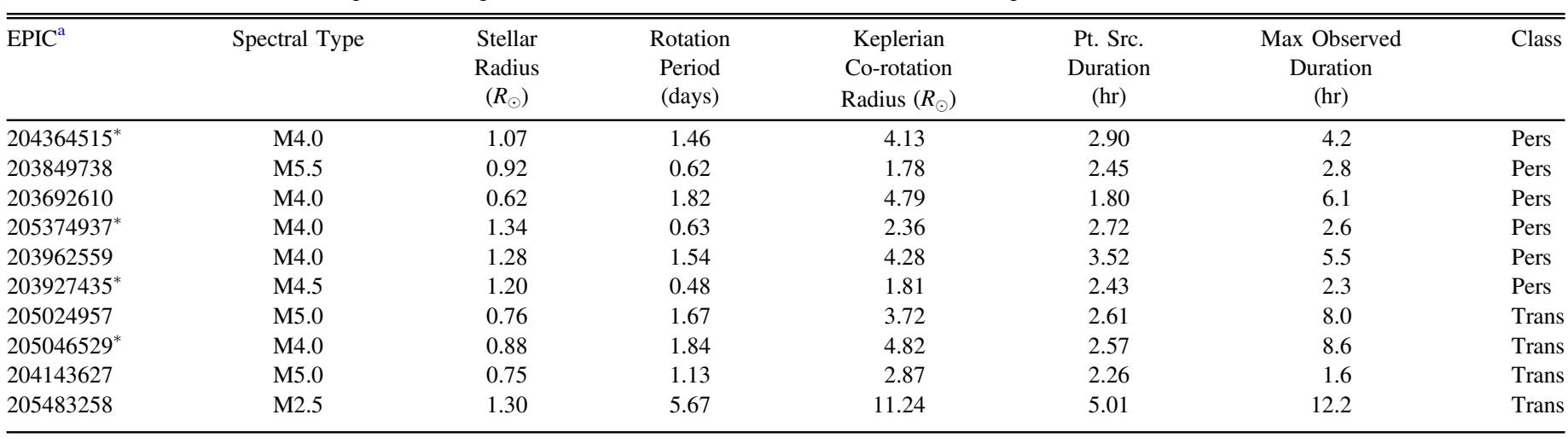

Note.

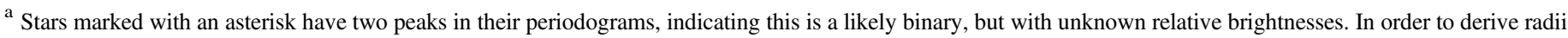
for the stars in these systems, we divided their luminosity estimates by 2 , corresponding to a reduction in their estimated radii and dip durations by 1.414 .

with inner rim at the Keplerian co-rotation radius, oriented so that the normal to the disk lies between the rotation and magnetic axes. The short-duration flux dips in the light curve arise when the disk transits our line of sight. Their model (see also Ud-Doula et al. 2006) also predicts break-out events, resulting when too much mass accumulates onto the field lines. These magnetic reconnections could resemble flares (which are otherwise not predicted for B stars).

While the Townsend \& Owocki (2005) model is for a highmass star with a radiative wind, it turns out that a very similar model had been proposed to explain some properties of rapidly rotating low-mass stars. Optical spectra of AB Doradus $(P=0.51$ days; Collier Cameron \& Robinson 1989a, 1989b) and Speedy Mic (HD 197890; $P=0.38$ days; Jeffries 1993; Dunstone et al. 2006) and other similar rapidly rotating $\mathrm{K}$ dwarfs show narrow $\mathrm{H} \alpha$ absorption dips that sweep through their broad quiescent $\mathrm{H} \alpha$ profiles, best explained as due to orbiting warm gas clouds at or above the Keplerian co-rotation radius that transit the observer's line of sight. Some Be stars show similar absorption dip transients (Smith \& Balona 2006). In AB Dor, often as many as six individual features (i.e., individual clouds) are seen at a given epoch. Many of the $\mathrm{H} \alpha$ transients repeat from night to night for periods up to the end of a week-long campaign (Dunstone et al. 2006). Similar features have also recently been detected in a rapidly rotating ( $P=0.44$ days) $\mathrm{M}$ dwarf, V374 Peg (Vida et al. 2016). Jardine \& van Ballegooijen (2005) proposed a model for these systems in which gas from the stellar (Parker) wind accumulates in a lumpy torus at or above the Keplerian co-rotation radius where net gravitational and magnetic forces vanish, with density maxima at the places where the radial component of the magnetic field changes polarity. These density maxima could be the "clouds" that give rise to the light-curve structure we see in the persistent flux-dip and scallop-shell light curves. Note that in this model, the flux dips in the $K 2$ data arise when one of these glowing clouds is eclipsed by the star, rather than when the cloud transits the face of the star and produces the $\mathrm{H} \alpha$ absorption transients. The scallop shells may differ from the persistent flux-dip class because they have an additional source of supra-photospheric gas-gas lost through their equatorial plane because they are rotating at breakup velocity.

The above speculations are at most proof of concept. The next step is to determine if the gas in these structures have densities, ionization fractions, and temperatures consistent with that needed to generate the light-curve features we see. We intend to address those issues in a future paper (A. Collier Cameron et al. 2017, in preparation).

\section{Summary and Conclusions}

By targeting the Upper Sco association, the second campaign of the $K 2$ mission provided the largest collection of highquality light curves for low-mass pre-main-sequence stars ever obtained. Those data made it possible to search for rare lightcurve types that only occur to a significant degree at very young ages and very low masses. We have, in fact, identified 23 stars with light-curve morphologies that have few or no previous counterparts in the literature. We have attempted to characterize these light curves as well as possible and to identify correlations or shared characteristics among these stars and their light curves that might point to the physical mechanism responsible for creating their unusual light-curve morphologies.

The most striking shared characteristics of the 19 stars in our two main groups are:

1. all have mid-to-late $\mathrm{M}$ dwarf spectral types;

2. more than half of the stars have rotation periods within a factor of two of the predicted breakup period;

3. eight of these stars show rapid "state-transitions" in their light-curve morphology, in many cases apparently triggered by a strong flare;

4. the short-duration flux dips and highly structured phased light curves seem incompatible with star spots as the driver of the photometric variability.

Warm gas clouds organized into a structured toroidal shape located at the Keplerian co-rotation radius seems to be the most plausible explanation for these characteristics. A discussion of a detailed physical model for creating and maintaining these clouds and for their producing our observed light-curve 
morphologies is provided in A. Collier Cameron et al. (2017, in preparation).

The four other Upper Sco stars we have identified have single short-duration flux dips superposed on normal spottedstar light curves, with the dip period and spot period being identical. The flux dips have highly variable depths and shapes, with shapes that are often asymmetric. These characteristics seem better matched to some type of dusty debris, orbiting a young planet located at or near the Keplerian co-rotation radius. A detailed examination of the best studied of this group (RIK-210) and a comparison of those data to a variety of physical models is provided in David et al. (2017).

While the unusual light-curve morphologies we find in Upper Sco may only be present for a small fraction of stars during a brief time interval in their early evolution, understanding these stars may have more far reaching implications. If an AB Dor slingshot prominence type model is found to be appropriate for the scallop-shell and persistent flux-dip morphologies, then analysis of those stars may provide important new clues to the magnetic field topologies, winds, and mass-loss rates of low-mass stars. These data could also help explain the comparatively uniform and rapid rotation found for most field brown dwarfs. If a young planet is present near the Keplerian co-rotation radius for the four transient flux-dip stars, that also could have significant implications for models of planet formation, particularly around $\mathrm{M}$ dwarfs.

We thank Leslie Hebb for timely advice on debugging and testing our spot simulation routine. We also thank Kevin Covey, Lee Hartmann, Nuria Calvet, Steve Saar, and Jeremy Drake for helpful discussions of these objects.

Some of the data presented in this paper were obtained from the Mikulski Archive for Space Telescopes (MAST). Support for MAST for non-HST data is provided by the NASA Office of Space Science via grant NNX09AF08G and by other grants and contracts. This paper includes data collected by the Kepler mission. Funding for the Kepler mission is provided by the NASA Science Mission directorate. This research has made use of the NASA/IPAC Infrared Science Archive (IRSA), which is operated by the Jet Propulsion Laboratory, California Institute of Technology, under contract with the National Aeronautics and Space Administration. This research has made use of NASA's Astrophysics Data System (ADS) Abstract Service, and of the SIMBAD database, operated at CDS, Strasbourg, France. This research has made use of data products from the Two Micron All-Sky Survey (2MASS), which is a joint project of the University of Massachusetts and the Infrared Processing and Analysis Center, funded by the National Aeronautics and Space Administration and the National Science Foundation. The 2MASS data are served by the NASA/IPAC Infrared Science Archive, which is operated by the Jet Propulsion Laboratory, California Institute of Technology, under contract with the National Aeronautics and Space Administration. This publication makes use of data products from the Wide-field Infrared Survey Explorer, which is a joint project of the University of California, Los Angeles, and the Jet Propulsion Laboratory/California Institute of Technology, funded by the National Aeronautics and Space Administration.

\section{Appendix A Spectral Energy Distributions}

SEDs for all of the stars from Table 1 are provided here as Figures 27-30. Plots are $\log \lambda F_{\lambda}$ in cgs units (ergs s $\mathrm{cm}^{2}$ ) against $\log \lambda$ in microns. Symbols: + : optical literature (SDSS, APASS); box at short bands: DENIS IJK; diamond: 2MASS $J H K_{s}$; circle: IRAC; box at long bands: MIPS; stars: WISE; arrows: limits; vertical bars (often smaller than the symbol) denote uncertainties. A Kurucz-Lejeune model for the corresponding spectral type (taken to be M3 for those without known types) is also shown as the grey line, normalized to the observations at $K_{s}$. Note that this is not a robust fit, but just to "guide the eye." Some models have been lightly reddened to better fit the optical points ( $A_{J} \leqslant 0.7$ in all cases). Almost all of the stars have SEDs consistent with pure photospheres; none have large IR excesses. EPICs 204117263 and 202873945 have apparent excesses, but the uncertainties are large and we do not believe these apparent excesses are real. EPIC 203534383 has a possible W3 detection but the PSF in the image is not circular, so we do not believe this is a secure excess. EPIC 203050730 has a W4 detection in the WISE catalog, but nothing is present at the source location in the image, so we have changed it to an upper limit. EPIC 204364515 and 205024957 both have possible W4 detections that are contradicted by the MIPS $24 \mu \mathrm{m}$ data; since MIPS is higher spatial resolution and more sensitive, we take the MIPS measurement as reality, rather than the W4. EPIC 205024957 has a small apparent excess at $24 \mu \mathrm{m}$ and it is the only reasonably secure IR excess for the stars in Table 1. Carpenter et al. (2009) had previously identified this star as having a $24 \mu \mathrm{m}$ excess, which they interpreted as due to a debris disk (because the shorter wavelength points are consistent with photospheric emission).

\section{Appendix B New High-resolution Spectra}

We have obtained high-resolution spectra for seven of the stars in Table 1 using the Keck HIRES spectrograph (Vogt et al. 1994). Additional HIRES spectra of one of these stars from Table 1-EPIC 205483258 (RIK-210) - are reported in David et al. (2017). The spectra cover the wavelength range roughly $4800-9200 \AA$, at a spectral resolution of about $R=45,000$. We measured equivalent widths for $\mathrm{H} \alpha$ and lithium using the SPLOT routine in IRAF.

For two of the stars-EPIC 203534383 and EPIC 203692610 - there was little spectral information in the published literature, and we have used the HIRES spectra to determine spectral types as well as to measure $\mathrm{H} \alpha$ and lithium equivalent widths. Figure 31 shows the $\mathrm{H} \alpha$ profiles for these two stars, and the Li $6708 \AA$ feature for EPIC 203692610; the $\mathrm{S} / \mathrm{N}$ at the lithium line for EPIC 203534383 is too poor to detect lithium. For EPIC 203692610, we determined equivalent widths of $-3.0 \AA$ for $\mathrm{H} \alpha$ and $0.55 \AA$ for $\mathrm{Li} 6708$. For EPIC 203534383, our derived $\mathrm{H} \alpha$ equivalent width is $-9.5 \AA$.

We determined spectral types for the two stars based on the strength of the TiO bandheads near $7100 \AA$ (Stauffer et al. 1979; Preibisch et al. 2001). For both stars, we estimate a spectral type of M4, though for EPIC 203534383 this is uncertain due to the low $\mathrm{S} / \mathrm{N}$ of the spectrum.

For the other four stars, EPIC 203849738, 204364515, 205024957, and 205046529, we only used the spectra to 

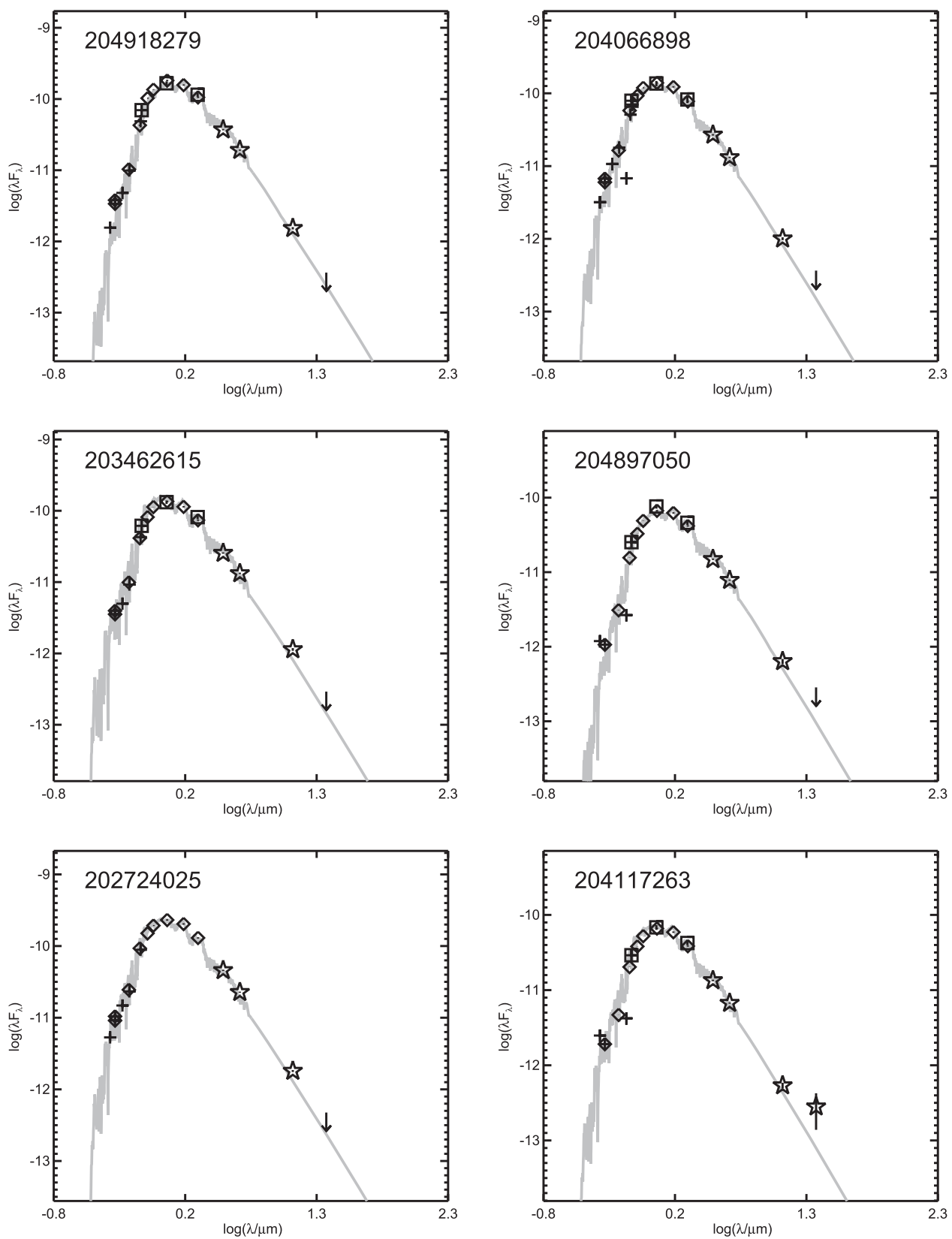

Figure 27. Spectral energy distributions for the first six stars in Table 1. See the text for a description of the symbols.

determine equivalent widths for the lithium $6708 \AA$ doublet. Those lithium equivalent widths are reported in Table 1.

\section{Appendix C}

\section{Phased Light-curve Library}

Phased light curves for all of the stars with scallop-shell light curves are provided in Figures 32 and 33. For those stars whose phased light morphology underwent significant evolution during the $K 2$ campaign, we split up the 78-day $K 2$ campaign into several time windows and plot each window with a different color point. Note that phase $=0$ is arbitrary for these light curves. Figure 34 shows median-smoothed versions of these light curves. For the stars whose phased morphology evolved during the $K 2$ campaign, we derived the median shape from only the first time window.
Figures 35 and 36 show similar figures for all stars in the persistent flux-dip class.

\section{Appendix D Light-curve Detrending}

Our standard processing (see Section 3) produced light curves from which short-term non-astrophysical trends had been removed and identified the best Lomb-Scargle period (or periods) for each star. Plots were created showing the original light curve and the light curve phased to each period identified for the star. Those latter plots were the basis for selecting stars that might be included in this paper.

In order to produce higher quality versions of the phased light curves and to validate stars for inclusion in the paper, we conducted additional processing. For stars with long-term 

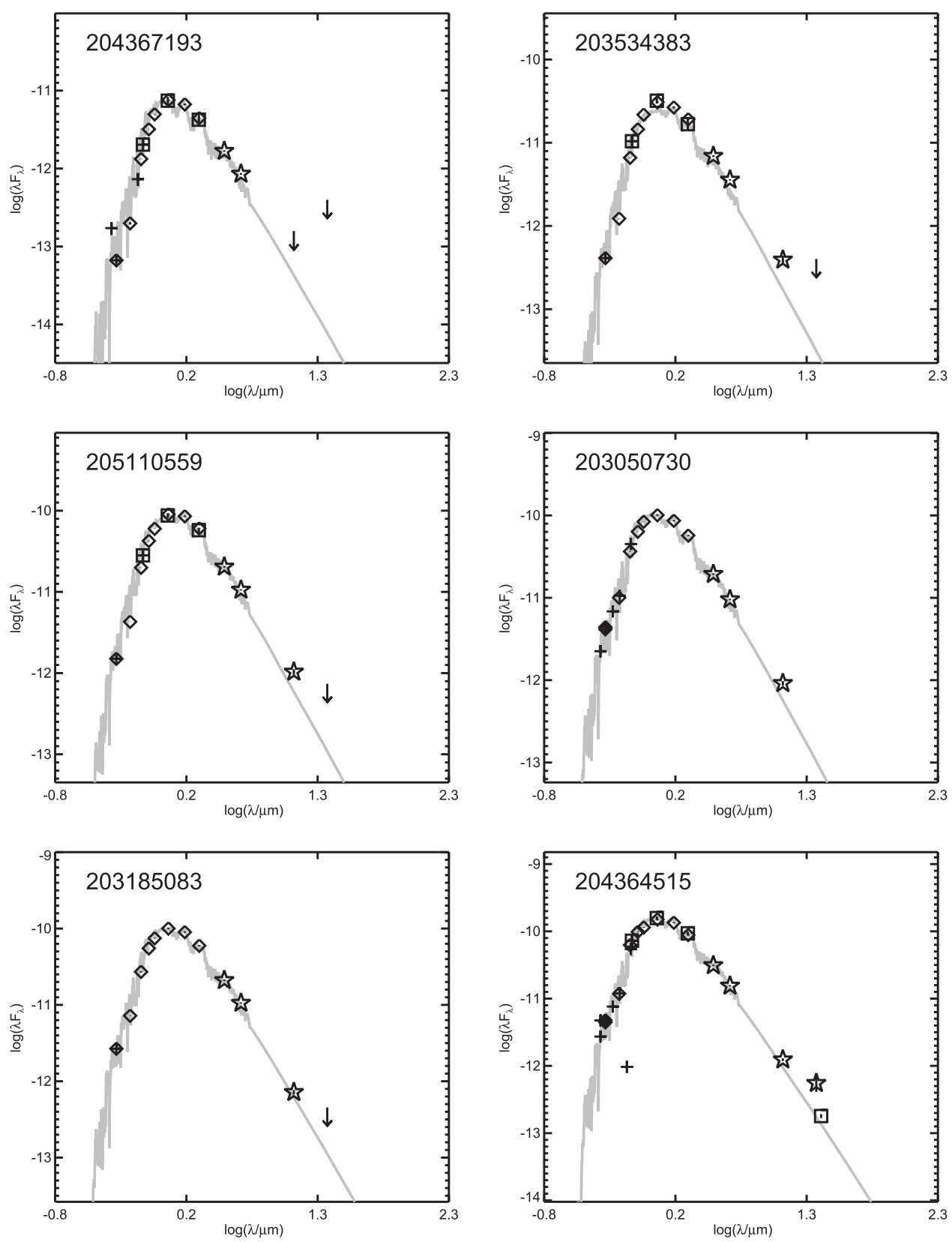

Figure 28. Spectral energy distributions for the next six stars in Table 1.

trends in their light curve, we fit low-order polynomials to the data and then removed the long-term trend (assuming that it was likely to be non-astrophysical-but that in any case the presence of the long-term trend would degrade the ability to phase-fold the light curve and examine its detailed structure). In some cases, even after this step, the light curve retained bumps and wiggles on timescales much longer than the rotation period but shorter than the $K 2$ campaign length. In those cases, we determined the median count rate at each period and then normalized the data in each period to the same rate (for stars with very short periods, we instead adopted an interval of four periods for the calculation). This again could remove real flux variations from the light curve, but removing these trends allowed us to produce phased light curves with significantly less noise.
More than half of the scallop-shell and persistent narrow flux-dip stars had two significant, narrow-peaked, independent (non-harmonic) Lomb-Scargle periods, indicating that they are binaries (or at least that light from two stars is contributing to the $K 2$ light curve). In all cases, the plots from our standard processing showed one of the two stars to be relevant for this paper with the other star appearing to have a normal, spottedstar light curve. For these systems, we adopted an iterative approach to the final processing. First, we phased the initial light curve to the period of the star that appears to have the larger light-curve amplitude. We then derived a mediansmoothed version of that light curve and subtracted that median-smoothed light curve from the initial light curveresulting in a residual light curve that should largely be the signal from the second star. We then phased that residual light 

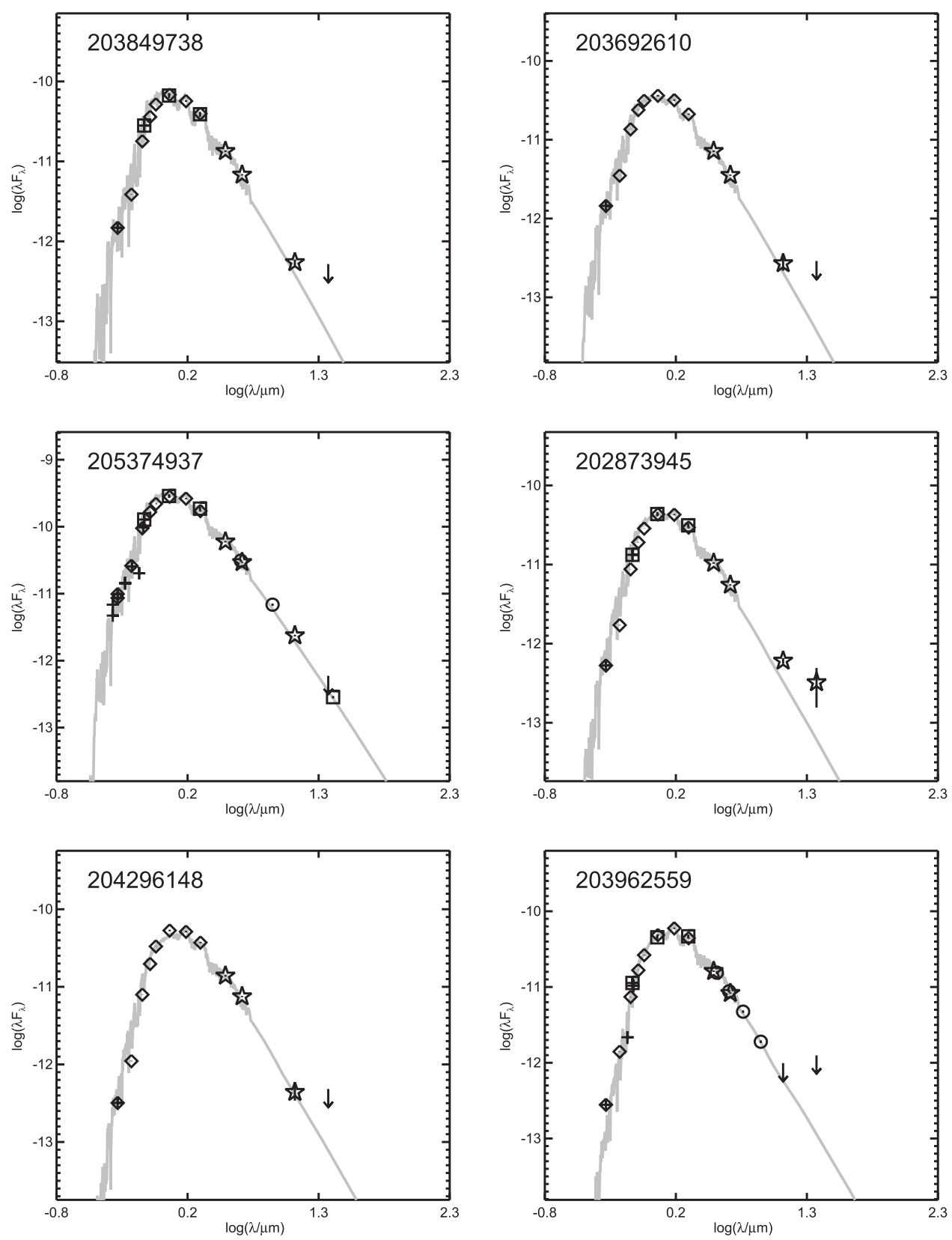

Figure 29. Spectral energy distributions for the next six stars in Table 1.

curve to the second Lomb-Scargle period, derived a mediansmoothed version of that, and then subtracted this mediansmoothed light curve from the initial light curve-resulting in a new residual light curve that should largely be the signal from the first star (now with the signal from the second star largely removed). This process was repeated if necessary-with the final product being a phased light curve for the star of interest, from which most or all of the signal from the binary companion had been removed.

\section{Appendix E \\ Simulated Light Curves of Spotted Stars}

Cool spots generally produce phased light-curve morphologies resembling sinusoids or broad dips or humps whose FWZI is approximately $50 \%$ in phase. That is because spots seen from most view angles will be visible for nearly half of the rotation period of the star (or more), and therefore the signatures they impose on the light curve will span half or more in extent of the phased light curve. However, for a view angle that places a spot very near the top or bottom of the visible hemisphere of the star, the spot could be visible for significantly less than half the rotation period. Therefore, it is conceivable that in such geometries, single spots could produce narrow flux dips (as observed for our persistent or transient flux-dip class) or that several spots at widely spaced longitudes could produce the highly structured, wavy phased light curves we find for the scallop-shell class.

To test this hypothesis, we created a program to produce simulated light curves for stars with up to four cool spots placed at arbitrary latitudes and longitudes, and viewed from an arbitrary view angle. The spots are required to be circular and 

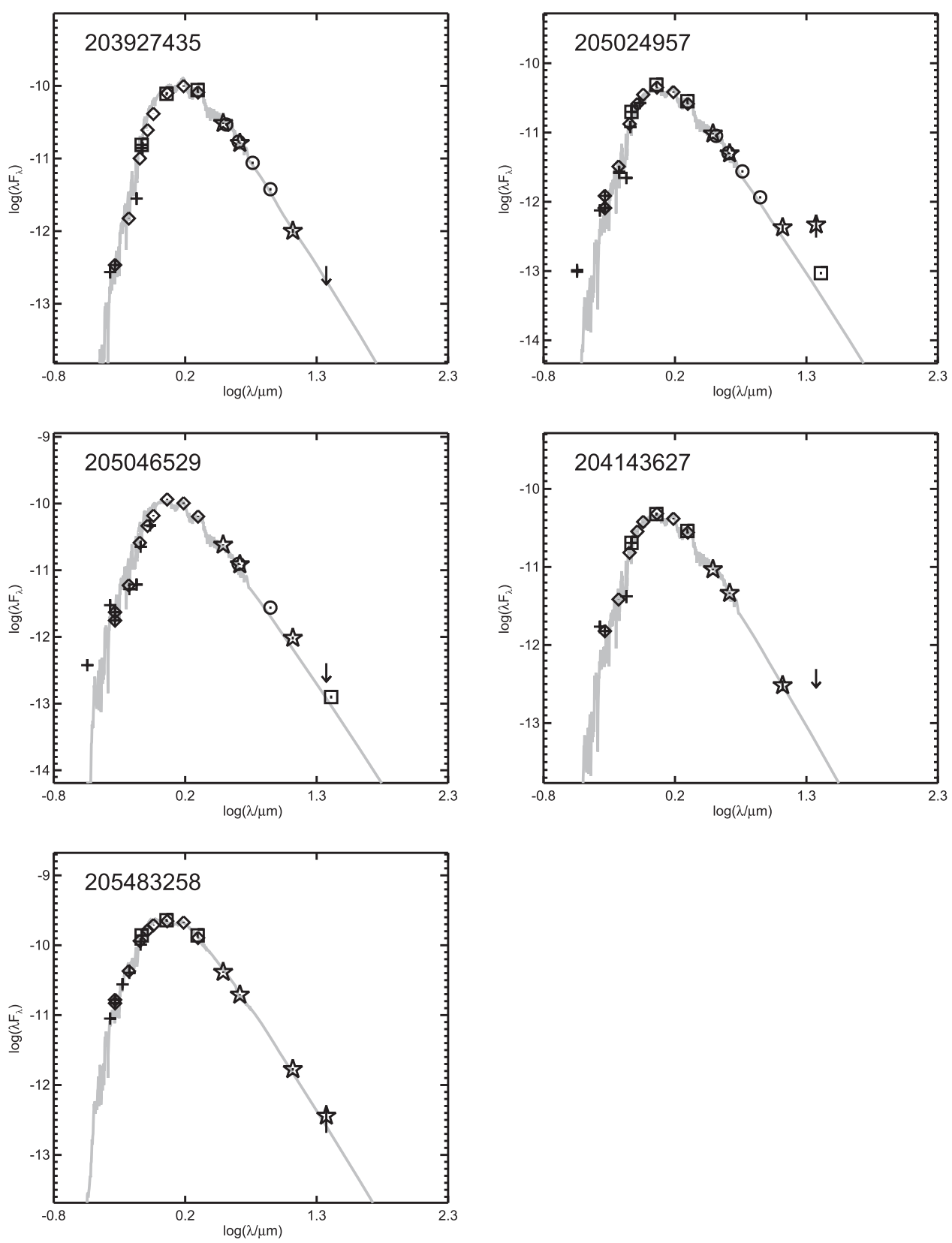

Figure 30. Spectral energy distributions for the remaining stars in Table 1.

to have a uniform surface brightness that is a factor of between 0.0 and 1.0 of the surface brightness of the pristine photosphere of the star. The star is assumed to be spherical. We adopt a linear limb darkening law and a limb darkening coefficient (normally designated as "u") of 0.8 , following the tables of Claret et al. (2012) for a mid-M dwarf and for the Kepler camera. We then allow the star to rotate and we measure its brightness after every 1 degree in rotation, following the star for a complete rotation period. For the present purposes, we set all the spots to be "black" - that is, non-emitting-because that will produce the largest possible amplitude for a given spot size.

We first used the program to attempt to produce narrow flux dips that might be similar to those we observe. The persistent flux-dip stars have FWZI for their dips ranging from 0.06 to 0.2 (in fractional phase) and depths ranging from $1 \%$ to $7.5 \%$, with an average of about $2.5 \%$. The transient flux-dip stars have FWZI for their dips of about $0.06,0.08,0.20$, and 0.20 and maximum depths of $1.5 \%, 2.5 \%, 3 \%$, and $20 \%$. To determine if those properties can be matched by cool spots, we first ran models with single spots of 8 degree radius, viewed from a range of locations up to a view angle that places the spot essentially at the upper limb of the visible hemisphere when the spot is facing the observer. Figure 37 shows the flux dips resulting from those spots for the selected viewing angles. Figure 38 shows how the width and depth of these flux dips vary with view angle.

Based on the two preceding figures, spots with radii of $8^{\circ}$ or less cannot be responsible for our short-duration flux dips because they are incapable of producing dip depths of $1 \%$ or more. We therefore decided to try a larger spot; specifically a spot of radius $18^{\circ}$. Figures 39 and 40 show the flux-dip 

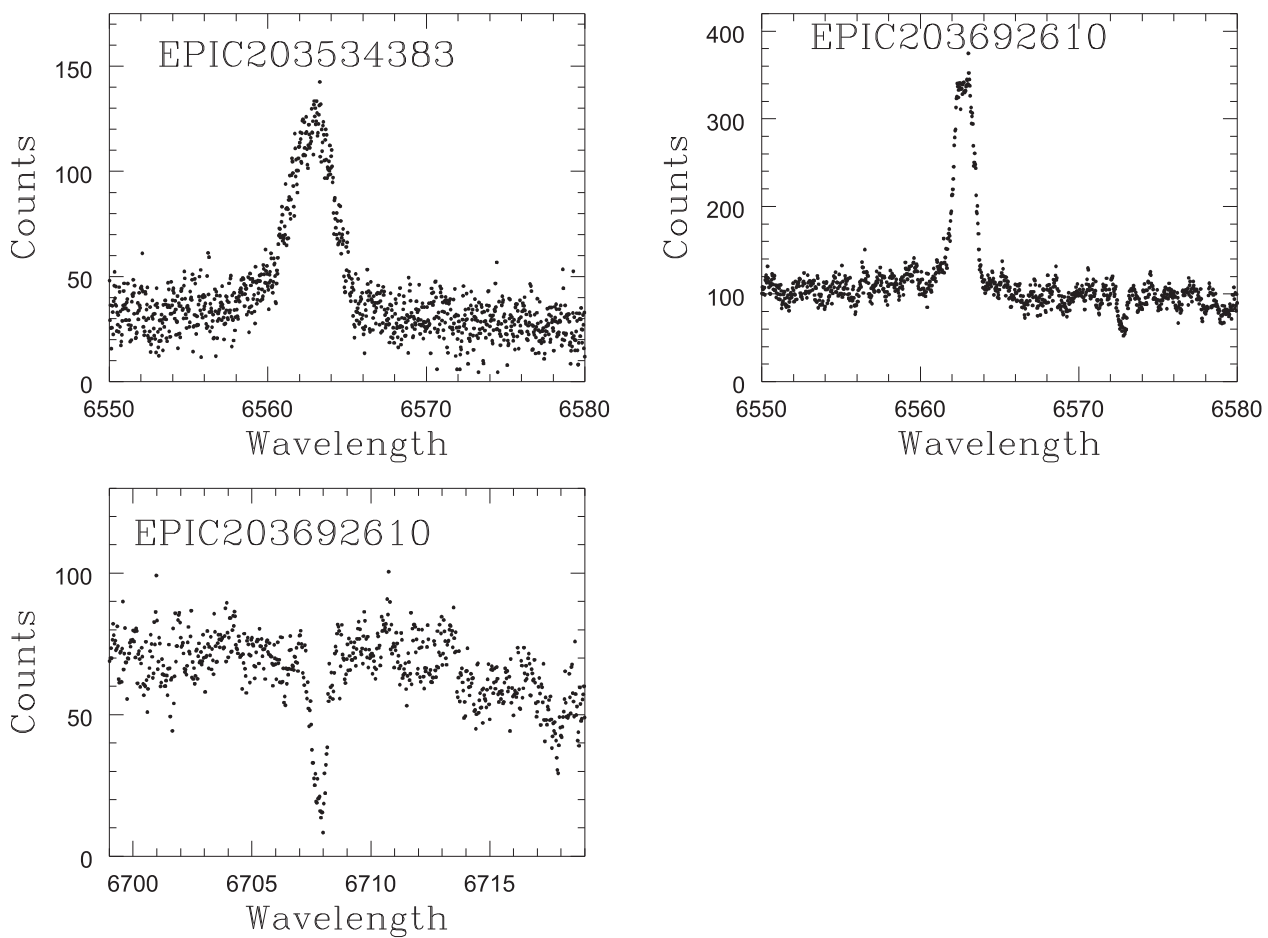

Figure 31. Snippets of HIRES spectra for $\mathrm{H} \alpha$ and for the lithium $6708 \AA$ A doublet for EPIC 203692610 and for just H $\alpha$ for EPIC 203534383.
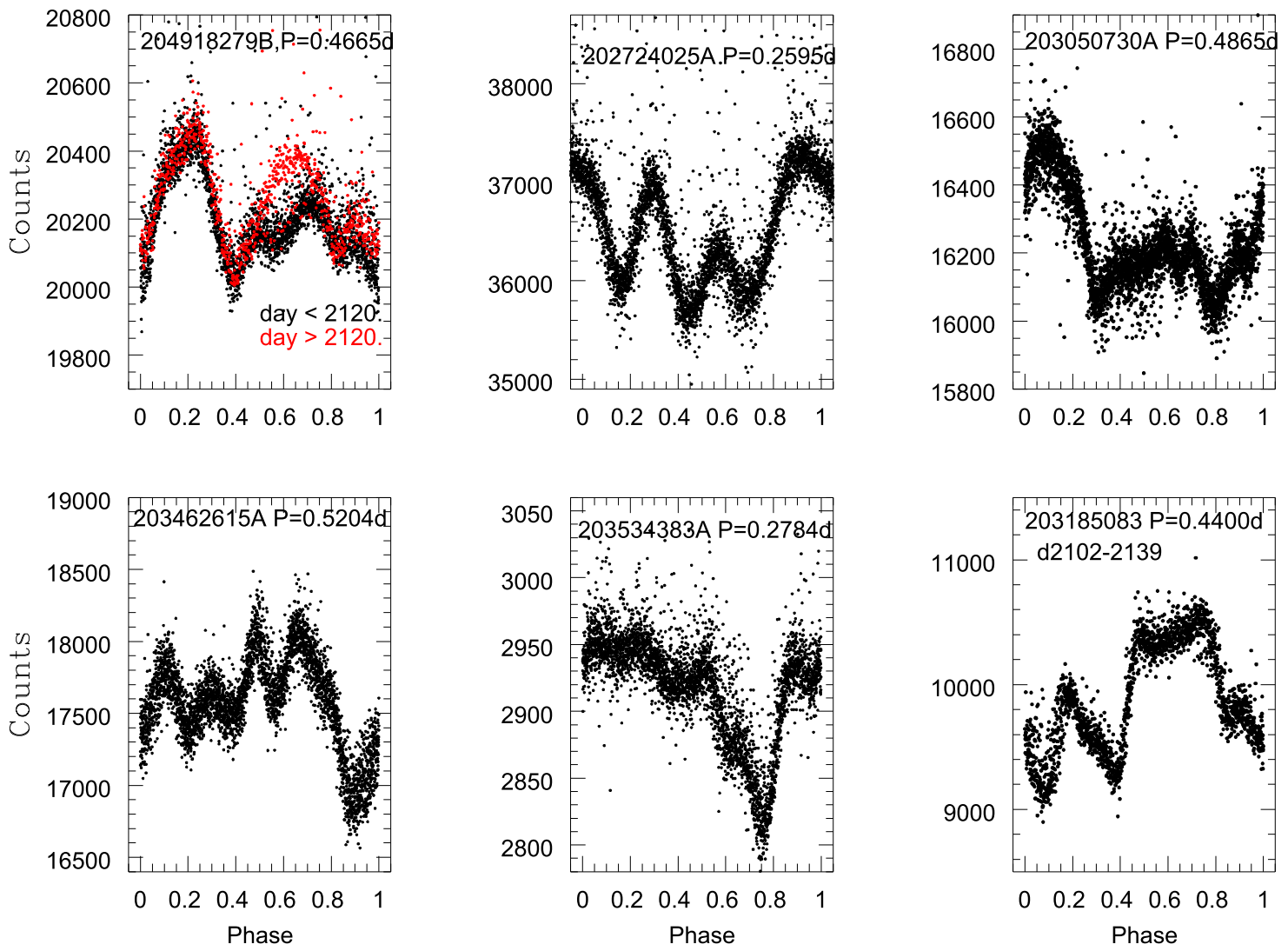

Figure 32. Phased light curves for six of the scallop-shell light-curve class.

shapes and their depth and width as a function of the observer's view to the star. A spot this size can produce fluxdip depths of several percent; however, the dips are relatively broad. The plots demonstrate that no view angle will produce a dip with depth $>1 \%$ and FWZI $\leqslant 0.2$. We have tried other combinations of parameters, but the answers are always the 

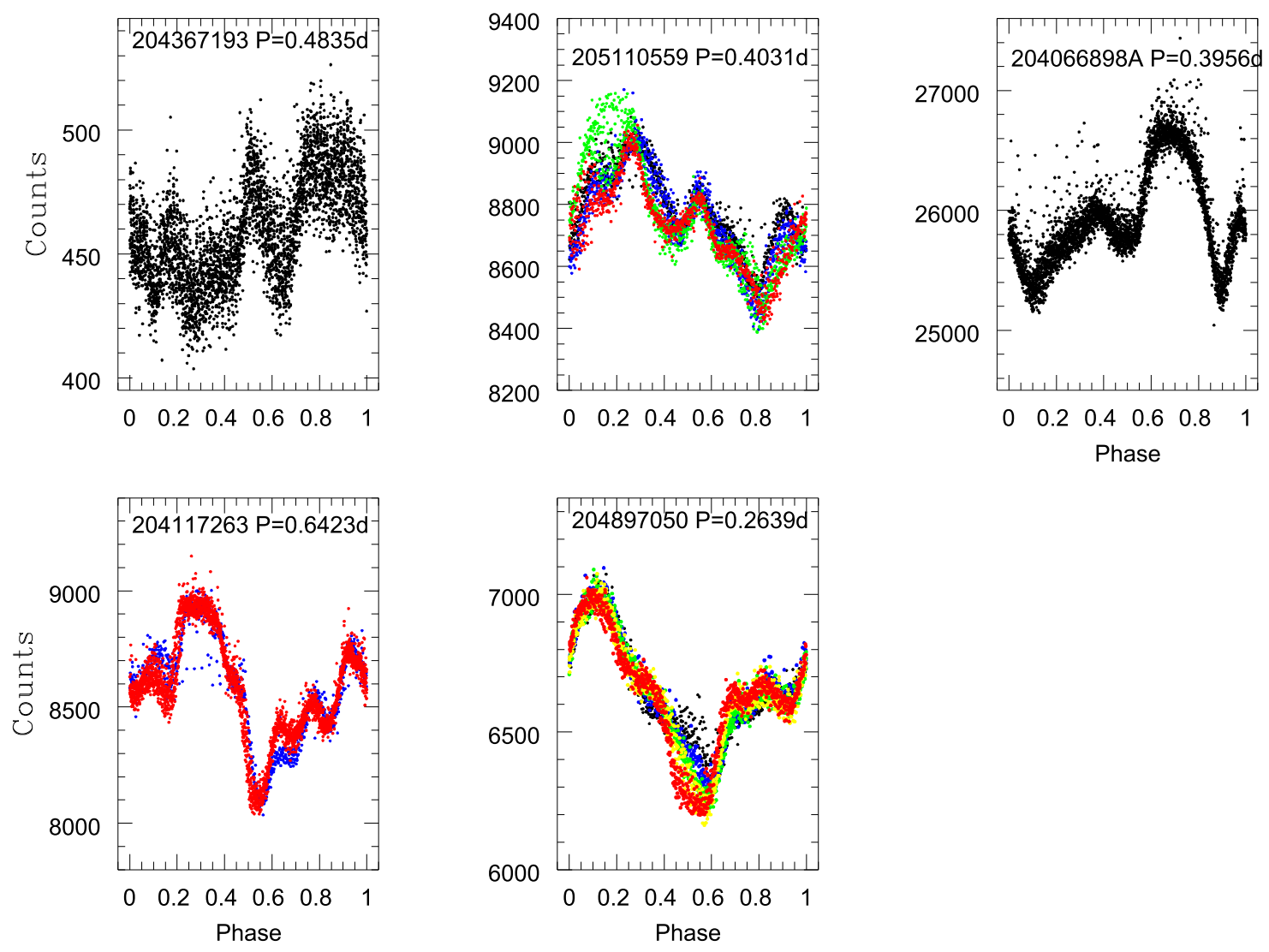

Figure 33. Phased light curves for five of the scallop-shell light-curve class.
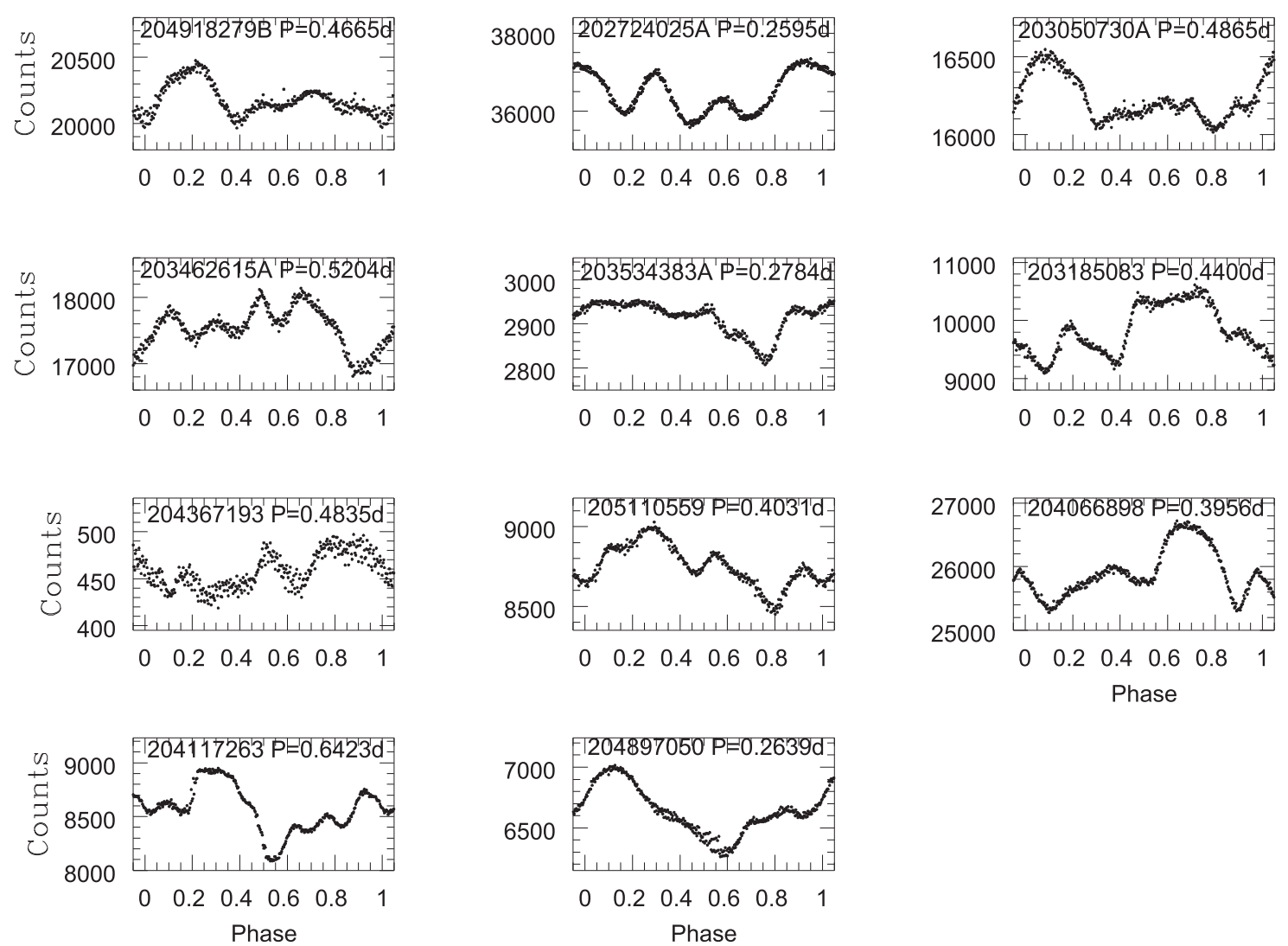

Figure 34. Median-smoothed phased light curves for stars in the scallop-shell class. Where the phased light curve changed shape significantly during the campaign, only data from either before or after the change was used in forming the median. 

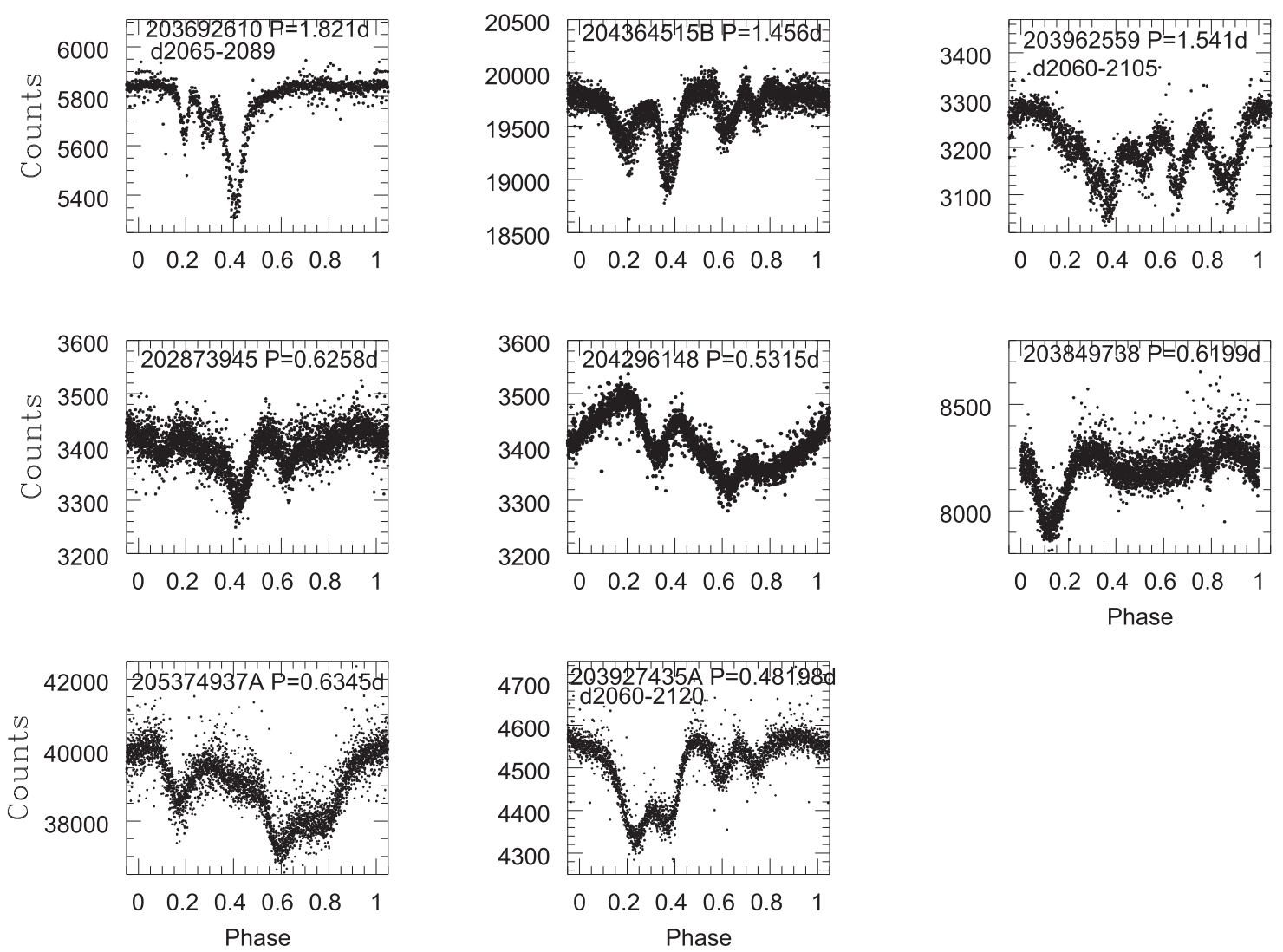

Figure 35. Phased light curves for stars in persistent flux-dip class.
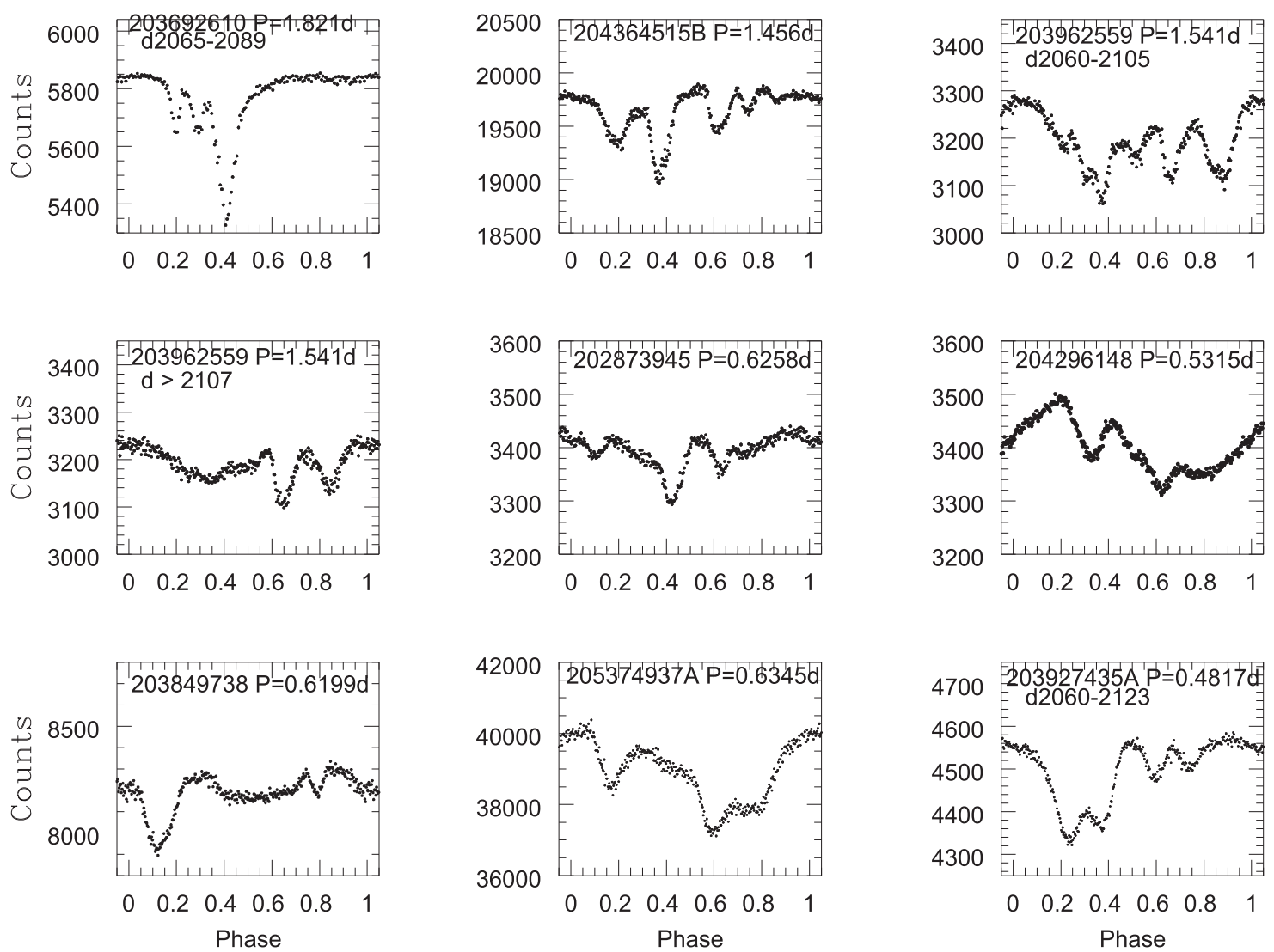

Figure 36. Median-smoothed phased light curves for stars in the persistent flux-dip class. EPIC 203962559 is shown twice in order to illustrate the change in its waveform after the flare on day 2106. 

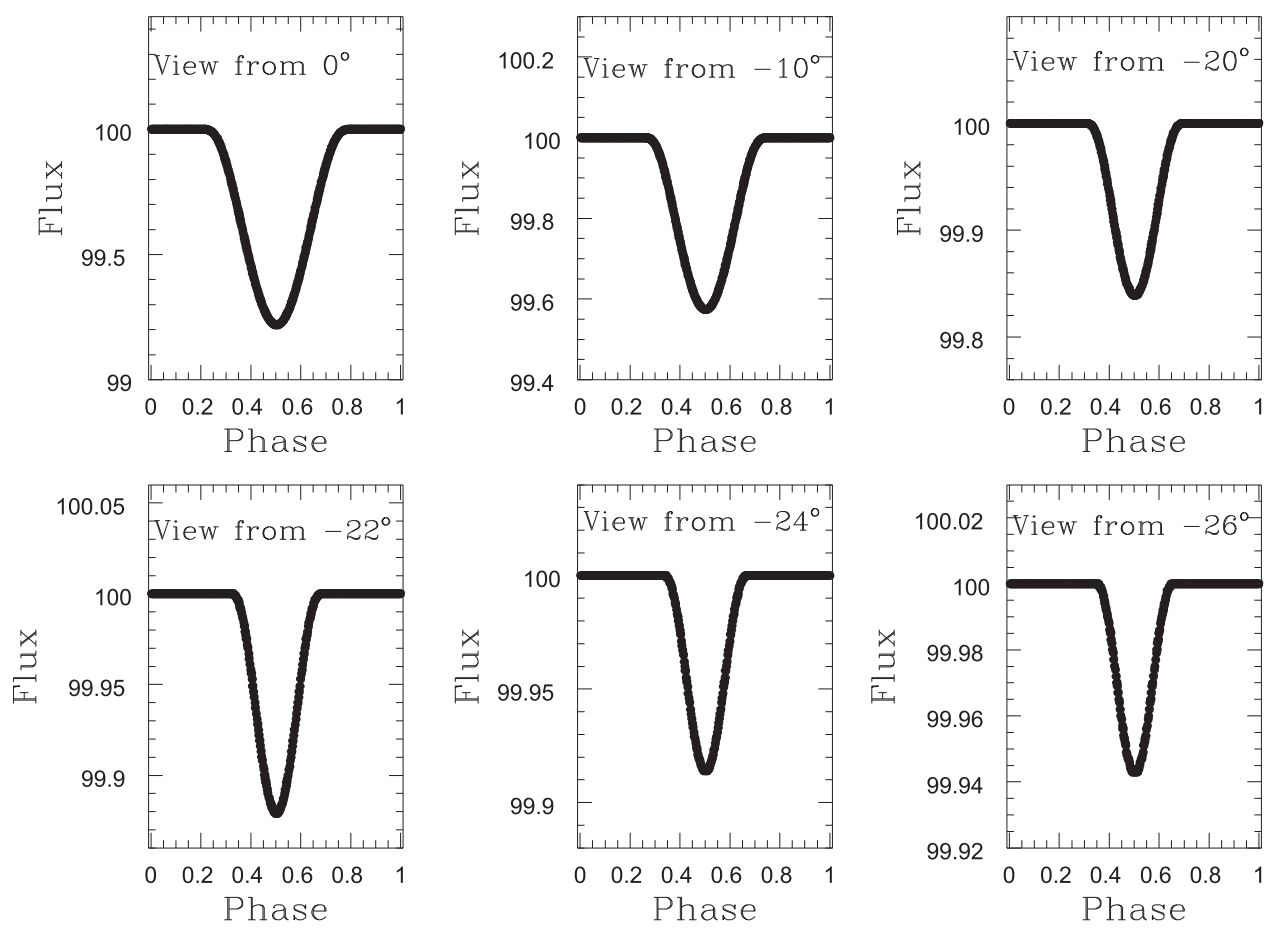

Figure 37. Model phased light curves for single spots of radius $8^{\circ}$. The spots are assumed to be circular and "black." The spot was placed at latitude $60^{\circ}$, with the viewer located at latitudes ranging from 0 to $-26^{\circ}$. Only the case for an observer at $-26^{\circ}$ produces a dip width comparable to the broadest dips we observe-but in that case, the dip depth is tiny.
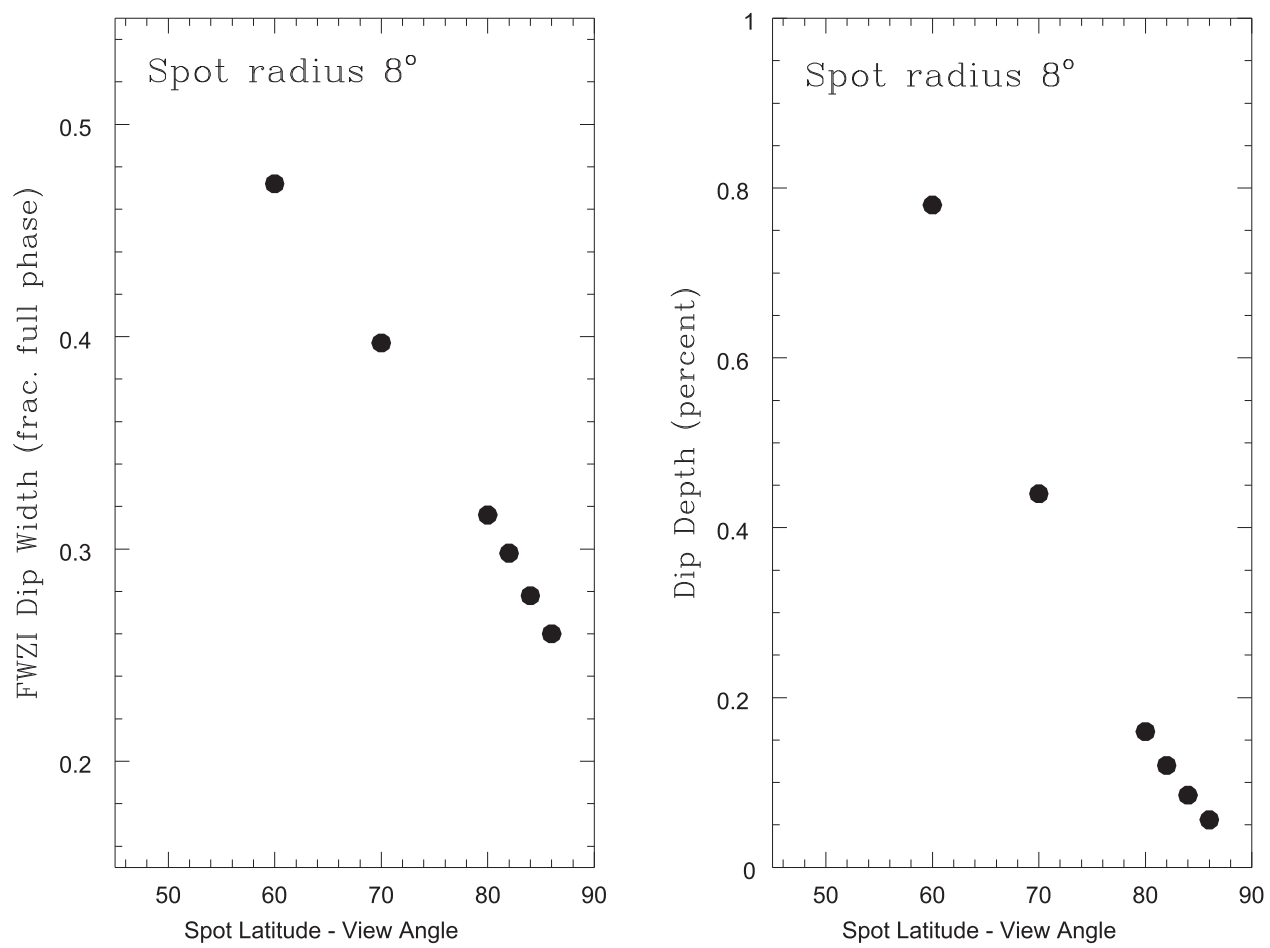

Figure 38. FWZI (in fraction of the full period) and dip depth (in percent) vs. the angle between the spot latitude and the observer latitude for the model stars with a single 8 degree radius spot. The plot shows that even for an angle of $60^{\circ}$, the dip will have a width covering almost half the phase. Only for angles $\sim 85^{\circ}$ do the widths approach what we observe in Upper Sco, but then the depths become $<0.1 \%$.

same. For any cold spot that can produce a FWZI of 0.2 or less, the spot depth will always be much less than $1 \% .^{16}$

\footnotetext{
${ }^{16}$ We actually measure the full width at $5 \%$ depth for the models, because they are noiseless. This more closely approximates the FWZI we measure for the observed light curves.
}

Therefore, cold spots cannot be the explanation for the shortduration flux dips for either our persistent flux-dip or transient flux-dip light curves.

As a test to determine if appropriately arranged spots could produce phased light curves resembling the scallop-shell class, we placed four spots of $8^{\circ}-10^{\circ}$ radius at latitudes between 60 and $62^{\circ}$ 

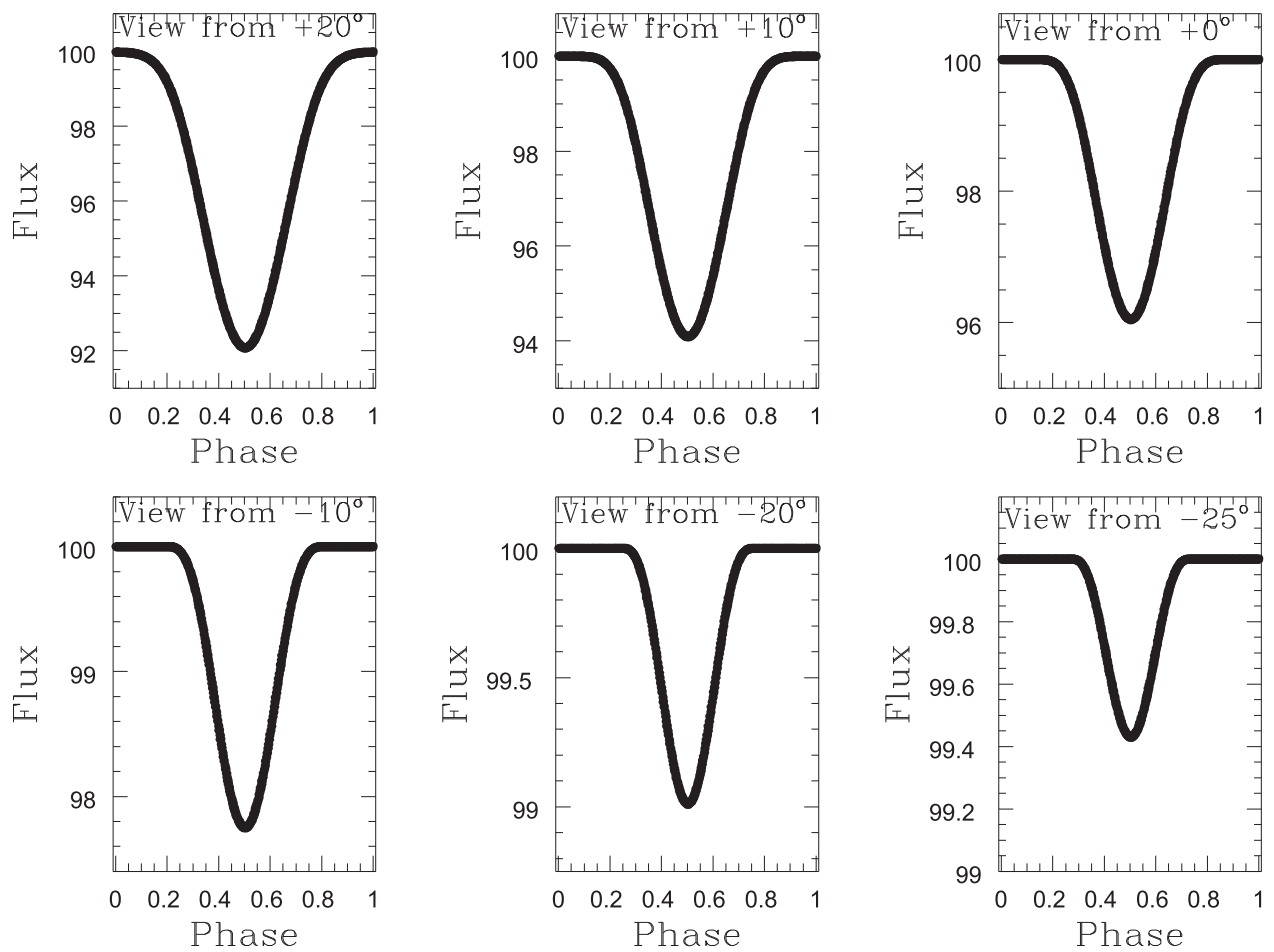

Figure 39. Model phased light curves for single spots of radius $18^{\circ}$. The spots are assumed to be circular and "black." The spot was placed at latitude $60^{\circ}$, with the viewer located at latitudes ranging from +20 to $-25^{\circ}$.
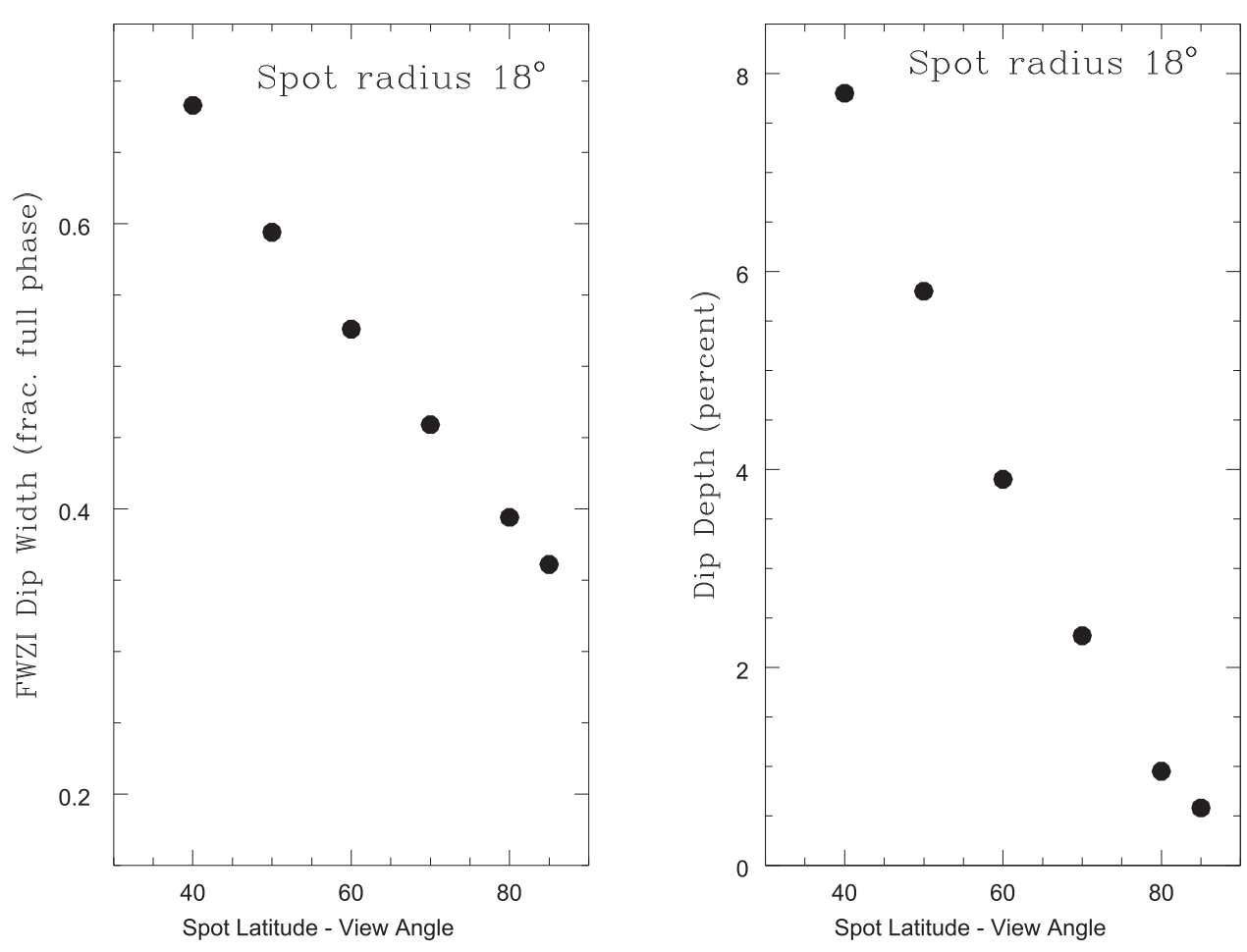

Figure 40. FWZI (in fraction of the full period) and dip depth (in percent) vs. the angle between the spot latitude and the observer latitude for the model stars with a single $18^{\circ}$ radius spot.

and longitudes of $0^{\circ}, 80^{\circ}, 180^{\circ}$, and $250^{\circ}$. We first put the observer at a latitude of $-24^{\circ}$ in order for those spots to be near the upper limb of the star as seen by the observer. As shown in the first panel of Figure 41, this does produce a phased light curve whose shape is reasonably similar to several of the stars in our scallop-shell class. However, the light-curve amplitude is tiny. The other panels in Figure 41 show that while the amplitude does increase as the observer to spot angle decreases, the degree of 

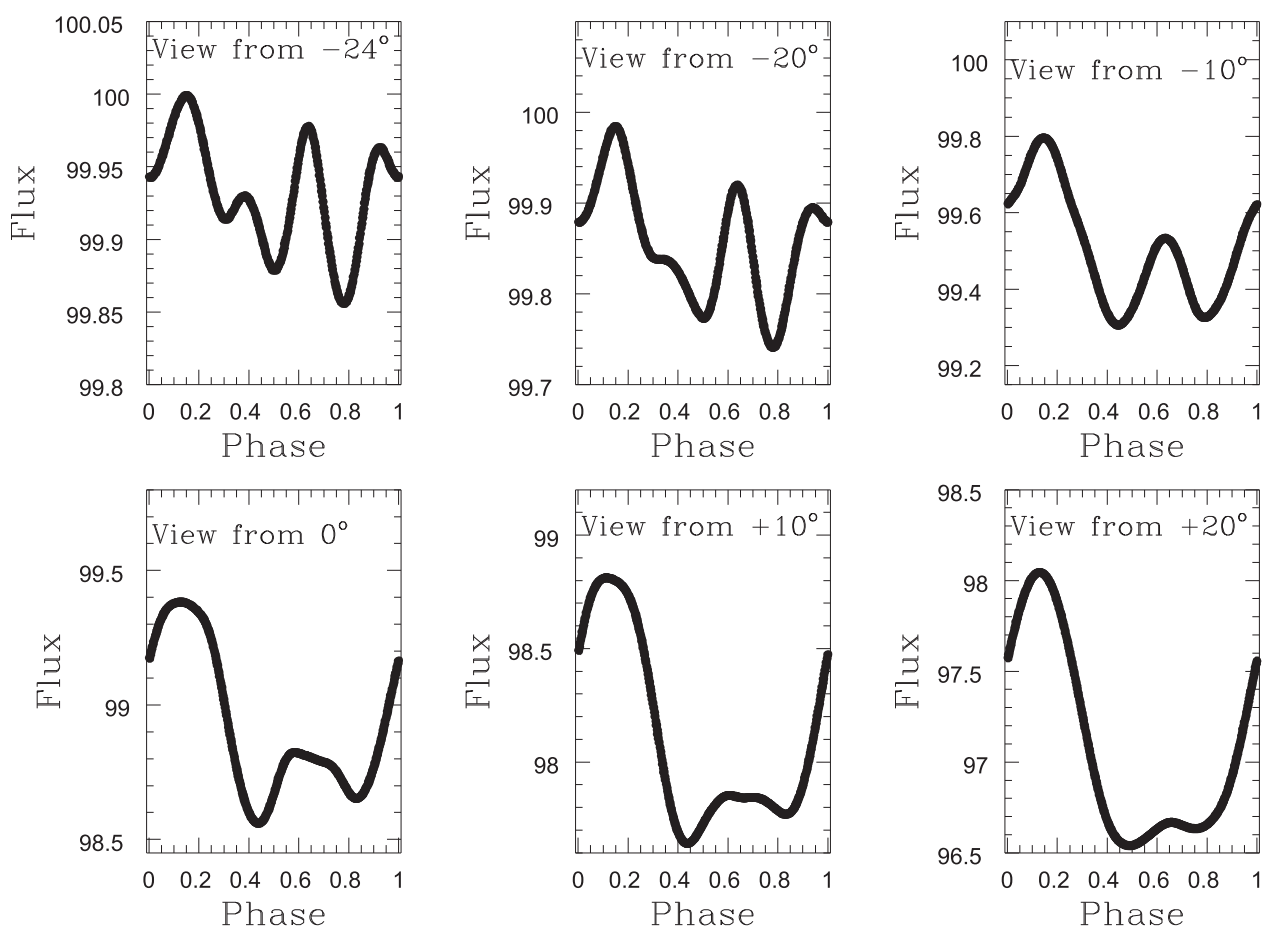

Figure 41. Model light curves for a star for four cold spots near $60^{\circ}$ latitude and widely spaced in longitude; the spots are assumed to have radii of $8^{\circ}-10^{\circ}$ and are completely black. When placed very near the upper limb of the star as seen by an observer, such an arrangement can produce a phased light curve whose shape is reminiscent of our scallop-shell class. However, the amplitude is tiny. At smaller view angles, the light-curve amplitude grows but the shape becomes progressively smoother.

structure in the light curve also decreases. By the point where the light-curve amplitude is $\sim 1.0 \%$, the morphology of the light curve is much smoother and no longer resembles the scallop-shell stars. Choosing larger spots does not help-there is again a trade off between degree of structure in the light curve and amplitude, and in no case have we been able to produce shapes that approximate our stars and had amplitudes $>0.4 \%$. Because many of the scallop-shell stars have light-curve amplitudes of 5\%-10\%, we conclude that cold spots also are unable to explain the scallopshell light-curve morphologies.

\section{References}

Ahn, C., Alexandroff, R., Allende Prieto, C., et al. 2014, ApJS, 211, 17 Aigrain, S., Parvianinen, H., \& Pope, B. 2016, MNRAS, 459, 2408 Alencar, S., Teixeira, P., Guimaraes, M., et al. 2010, A\&A, 519, 88 Alonso, R., Deeg, H., Hoyer, S., et al. 2015, A\&A, 584, L8 Alves de Oliveira, C., \& Casali, M. 2008, A\&A, 485, 155 Ansdell, M., Gaidos, E., Mann, A., et al. 2016, ApJ, 816, 69 Ardila, D., Martin, E., \& Basri, G. 2000, AJ, 120, 479 Baraffe, I., Homeier, D., Allard, F., \& Chabrier, G. 2015, A\&A, 577, 42 Bouvier, J., Chelli, A., Carrasco, L., et al. 1999, A\&A, 349, 619 Bouy, H., Moraux, E., Bouvier, J., et al. 2006, ApJ, 637, 1056 Boyajian, T., LaCourse, D., Rappaport, S., et al. 2016, MNRAS, 457, 3988 Carpenter, J., Bouwman, J., Mamajek, E., et al. 2009, ApJS, 181, 197 Claret, A., Hauschildt, P., \& Witte, S. 2012, A\&A, 546, 14 Cody, A., Hillenbrand, L., David, T., et al. 2017, ApJ, 836, 41 Cody, A., Stauffer, J., Baglin, A., et al. 2014, AJ, 147, 82 Collier Cameron, A., \& Robinson, R. 1989a, MNRAS, 236, 57 Collier Cameron, A., \& Robinson, R. 1989b, MNRAS, 238, 657 David, T., Hillenbrand, L., Cody, A., et al. 2016a, ApJ, 816, 21 David, T., Hillenbrand, L., Cody, A., et al. 2017, ApJ, 835, 168 David, T., Hillenbrand, L., Petigura, E., et al. 2016b, Natur, 534, 658 Dunstone, N., Collier Cameron, A., Barnes, J., \& Jardine, M. 2006, MNRAS, 373,1308

Epchtein, N., de Batz, B., Capoani, L., et al. 1997, Msngr, 87, 27
Erickson, K., Wilking, B., Meyer, M., et al. 2011, AJ, 142, 140 Flewelling, H., Magnier, E., Chambers, K., et al. 2016, arXiv:1612.05243 Gaia Collaboration, Prusti, T., de Bruijne, J. H. J., et al. 2016, A\&A, 595, A2 Henden, A., \& Munari, U. 2014, CoSka, 43, 518

Howell, S., Sobeck, C., Haas, M., et al. 2014, PASP, 126, 398 Huber, D., Bryson, S., Haas, M., et al. 2016, ApJS, 224, 2

Jardine, M., \& van Ballegooijen, A. 2005, MNRAS, 361, 1173

Jeffries, R., Jackson, R., Franciosini, E., et al. 2016, arXiv:1609.07150 Jeffries, R. D. 1993, MNRAS, 262, 369

Johns-Krull, C., Prato, L., McLane, J., et al. 2016, ApJ, 830, 15

Kraus, A., Cody, A., Covey, K., et al. 2015, ApJ, 807, 3

Kraus, A., \& Hillenbrand, L. 2009, ApJ, 703, 1511

Kraus, A., \& Hillenbrand, L. 2012, ApJ, 757, 141

Lafreniere, D., Jayawardhana, R., van Kerkwijk, M., et al. 2014, ApJ, 785, 47 Lodieu, N., Alonso, R., Gonzalez Hernandez, J., et al. 2015, A\&A, 584, 128 Lodieu, N., Dobbie, P., \& Hambly, N. 2011, A\&A, 527, 24

Lodieu, N., Hambly, N., Jameson, R., et al. 2007, MNRAS, 374, 372

Luger, R., Agol, E., Kruse, E., et al. 2016, AJ, 152, 100

Luhman, K., \& Mamajek, E. 2012, ApJ, 758, 31

MacDonald, J., \& Mullan, D. 2013, ApJ, 765, 126

MacDonald, J., \& Mullan, D. 2017, ApJ, 834, 67

Mann, A., Newton, E., Rizzuto, A., et al. 2016, AJ, 152, 61

McGinnis, P., Alencar, S., \& Guimaraes, M. 2015, A\&A, 577, 11

McQuillan, A., Aigrain, S., \& Mazeh, T. 2013, MNRAS, 432, 1203

Meyer, M., Hildebrand, L., Backman, D., et al. 2006, PASP, 118, 1690

Monnier, J., \& Millan-Gabet, R. 2002, ApJ, 579, 694

Morales-Calderon, M., Stauffer, J., Hillenbrand, L., et al. 2011, ApJ, 733, 50

Murakami, H., Baba, H., Barthel, P., et al. 2007, PASJ, 59, 369

Papaloizou, J. 2007, A\&A, 463, 775

Pecaut, M., \& Mamajek, E. 2013, ApJS, 208, 9

Preibisch, T., Brown, A., Bridges, T., et al. 2002, AJ, 124, 404

Preibisch, T., Guenther, E., \& Zinnecker, H. 2001, AJ, 121, 1040

Rappaport, S., Levine, A., Chiang, E., et al. 2012, ApJ, 752, 1

Rebull, L., Padgett, D., McCabe, C., et al. 2010, ApJS, 186, 259

Rebull, L., Stauffer, J., Bouvier, J., et al. 2016, AJ, 152, 114

Rizzuto, A., Ireland, M., \& Kraus, A. 2015, MNRAS, 448, 2737

Sanchis-Ojeda, R., Rappaport, S., Palle, E., et al. 2015, ApJ, 812, 112

Skrutskie, M., Cutri, R. M., Stiening, R., et al. 2006, AJ, 131, 1163

Slesnick, C., Hillenbrand, L., \& Carpenter, J. 2008, ApJ, 688, 377 
Smith, M., \& Balona, L. 2006, ApJ, 640, 491

Somers, G., \& Stassun, K. 2017, AJ, 153, 101

Stauffer, J., Cody, A., McGinnis, P., et al. 2015, AJ, 149, 130

Stauffer, J., Rebull, L., Bouvier, J., et al. 2016, AJ, 152, 115

Stauffer, J., Spinrad, H., \& Thorstensen, J. 1979, PASP, 91, 59

Townsend, R., \& Owocki, S. 2005, MNRAS, 357, 251

Townsend, R., Rivinius, Th., Rowe, J., et al. 2013, ApJ, 769, 33

Ud-Doula, A., Townsend, R., \& Owocki, S. 2006, ApJL, 640, L19

van Eyken, J., Ciardi, D., van Braun, K., et al. 2012, ApJ, 755, 42
Vanderburg, A., \& Johnson, J. 2014, PASP, 126, 948

Vanderburg, A., Johnson, J., Rappaport, S., et al. 2015, Natur, 526, 546

Venuti, L., Bouvier, J., Cody, A., et al. 2017, A\&A, 599, A23

Vida, K., Kriskovics, L., Olah, K., et al. 2016, A\&A, 590, 11

Vogt, S., Allen, S., Bigelow, B., et al. 1994, Proc. SPIE, 2198, 362

Werner, M., Roellig, T., Low, F., et al. 2004, ApJS, 154, 1

Wilking, B., Meyer, M., Robinson, J., \& Green, T. 2005, AJ, 130, 1733

Wright, E., Eisenhardt, P. R. M., Mainzer, A. K., et al. 2010, AJ, 140, 1868 